\title{
Focal Adhesion Kinase as a Cellular Mechano-Sensor
}

Jing Zhou

Molecular Biomechanics

Heidelberg Institut für Theoretische Studien

$\&$

Ruprecht-Karls-Universität Heidelberg

This dissertation is submitted for the degree of

Dr. rer. nat.

18 February, 2015 



\title{
INAUGURAL - DISSERTATION
}

\author{
Zur
}

\author{
Erlangung der Doktorwürde
}

der

Naturwissenschaftlich-Mathematischen

\author{
Gesamtfakultät
}

der

\section{Ruprecht-Karls-Universität \\ Heidelberg}

vorgelegt von

Diplom-Chemikerin Jing Zhou

aus Shanghai, V.R.China

Tag der mündlichen Prüfung: 18.02.2015 


\section{Focal Adhesion Kinase as a Cellular Mechano-Sensor}


To my family! 



\section{ACKNOWLEDGEMENTS}

First and foremost, I would like to express the deepest gratitude to my supervisor Prof. Dr. Frauke Gräter for offering me the challenging task, for being always open for discussions, and for her patience and constant encouragement. Her immense knowledge and insightful suggestions guided me throughout the course of this thesis, meanwhile, she also encouraged me to solve problems with my own ideas. Without her supervision and constant help this dissertation would not have been possible. I also would like to thank Prof. Dr. Joachim P. Spatz for taking over the role of my primary referee and giving me the opportunity to work on this topic. Thanks to BMBF Systec project and Klaus Tschira foundation for the financial support.

Special thanks to Agnieszka and Bogdan for all the helpful suggestions, discussions and teaching throughout this thesis. Thanks to Camilo for his support and explanations these last months. I am very grateful to Bogdan, Maxime and Cedric for their technical support. Many thanks to Agnieszka, Davide and Katra for correcting the manuscript of this thesis. I would like to thank the entire Molecular Biomechanics group for providing a nice atmosphere for research and the after-hour distractions from science. I will never forget the funny and extraordinary topics during lunch time. Extended thanks to all the HITSters, who made this institute a friendly and lovely working place.

I also would like to express many thanks to Dr. Sahle Sven in Bioquant, Heidelberg, for his patience and support for building the biochemical signaling network and Prof. Dr. Daniel Lietha at CNIO, Madrid for helpful discussions and our fruitful collaboration.

I also owe thanks to my Chinese friends in Heidelberg who share the happiness, laughing and joy with me and to my best friend, Bo Zhang, for her constant support and encouragement over the years. Their company is the power to push me to do better and go further. 


\section{Acknowledgements}

Most importantly, I would like to thank my parents for their constant and unconditional support, encouragement and understanding. Even though we are apart, they are always behind me. 


\section{ABSTRACT}

Mechanical signaling plays a crucial role in cell communication. Translating mechanical force into biochemical reactions is vital for virtually all cellular processes such as cell proliferation and differentiation. Mechanosensors, which can respond to an external force in form of controllable conformational changes, are key regulators in the mechanical signal transduction processes. In this work, we present results from Molecular Dynamics (MD) and biochemical network simulations that suggest Focal Adhesion Kinase (FAK) as a mechanosensing enzyme in the signaling pathway.

FAK is a non-receptor tyrosine kinase, located at the cytoplasmic site of the cell membrane. During the last decade, a growing body of evidence shows that tensile stress acting on cells leads to increased FAK activity. However, the mechanism of FAK mechanical activation has not been resolved. The auto-inhibitory conformation of the FAK FERMkinase fragment suggests that the major mechanism of the regulation of FAK activity is the release of Tyr576/577 on the activation loop from the N-terminal FERM domain. We first addressed the allosteric regulation of the FAK by $\mathrm{PIP}_{2}$ binding (Chapter 3), a recently recognized stimulus for FAK activation, by performing all-atom MD simulations of FAK FERM-kinase fragment and comparing the dynamics in absence and presence of ATP and $\mathrm{PIP}_{2}$. A closing-opening motion between the kinase and FERM domain upon ATP and additional $\mathrm{PIP}_{2}$ binding was observed in close agreement with corresponding changes in fluorescence resonance energy transfer experiments. As an underlying allosteric mechanism, using Force Distribution Analysis (FDA), a signal network spanning from the $\mathrm{PIP}_{2}$ and ATP binding site to the distant interface between the FERM and kinase domains could be identified. However, our results also demonstrated that the ligand induced conformational changes are insufficient for FAK catalytic turnover, which requires full exposure of Tyr576/577. This suggests that an additional biochemical or mechanical stimulus is required 
for FAK activation.

Following the study of FAK allosteric regulation, we suggest a mechanical model of FAK activation in which tensile forces, propagating from the membrane through the $\mathrm{PIP}_{2}$ binding site of the FERM domain and from the cytoskeleton-anchored FAT domain, activate FAK by relieving the occlusion of the central phosphorylation site of FAK (Tyr576/577). To test the hypothesis of FAK as a force-sensor, extensive Force Probe Molecular Dynamics (FPMD) simulations, with varying loading rates, pulling directions and membrane $\mathrm{PIP}_{2}$ concentrations, were carried out. They directly supported the notion of a specific force-induced opening and activation of FAK. This is remarkable given that force-induced unfolding of the primarily $\alpha$-helical FERM and kinase domains are competitive processes but were observed to be less favored over domain-domain dissociation. To assess downstream consequences of FAK mechano-sensing, force-dependent FAK kinetics based on extrapolated MD data were implemented as parameters to establish a mechano-biochemical network model, which connects force-dependent FAK activation to the downstream exchange rate of GTP/GDP in Ras. Our computational study provides direct evidence for FAK-mediated mechanical signaling through Ras to the nucleus, with predictions directly testable by cell stretching experiments and single molecule force spectroscopy. 


\section{ZUSAMMENFASSUNG}

Die mechanische Weiterleitung von Signale spielt in der Zellkommunikation eine entscheidende Rolle. Die Übertragung von mechanischer Kraft in biochemische Reaktionen ist die Grundlage für nahezu alle zellulären Prozesse wie Zelldifferenzierung und Zellteilung. Mechanosensoren, die in Form von kontrollierbaren Konformationsänderungen auf eine externe Kraft reagieren können, sind die Schlüsselregulatoren in mechanischen Signalübertragungsprozessen. Im Rahmen dieser Arbeit präsentieren wir Ergebnisse aus Molekulardynamik (MD) und biochemischen Netzwerksimulationen, welche auf eine Rolle der Fokalen Adhäsionskinase (FAK) als ein bedeutendes mechano-signalisierendes Enzym innerhalb des Signalweges hindeuten.

Die Fokale Adhäsionskinase (FAK) ist eine Nicht-Rezeptor-Tyrosinkinase, die sich an der zytoplasmatischen Seite der Zellmembran befindet. Innerhalb des letzten Jahrzehnts findet sich eine steigende Anzahl von Belegen dafür, dass die Einwirkung von Zugspannung auf Zellen zu einer Steigerung der FAK-Aktivität führt. Allerdings sind die Mechanismen dieser mechanischen Aktivierung der FAK noch nicht geklärt. Die auto-inhibitorische Konformation des FAK FERM-Kinase-Fragments von FAK legt nahe, dass die Hauptmechanismen der Regulation der FAK-Aktivität in der Freisetzung von Tyr576/577 auf der Aktivierungsregion von der N-terminalen FERM-Domäne bestehen. Wir haben zunächst eine allosterische Regulation der FAK durch $\mathrm{PIP}_{2}$ Bindung untersucht (Kapitel 3). Bei $\mathrm{PIP}_{2}$ handelt es sich um einen kürzlich erkannten Stimmulus zur FAK-Aktivierung. In alle Atome umfassenden MD-Simulationen eines FAK FERM-Kinase-Fragments konnten wir die Dynamik in Anwesenheit und Abwesenheit von ATP und $\mathrm{PIP}_{2}$ untersuchen. Es wurde eine schließende und öffnende Bewegung zwischen der Kinase und FERM bei Anwesenheit von ATP und zusätzlicher PIP 2 -Bindung beobachtet, was die entsprechenden Änderungen in den Experimenten zum Fluoreszenz-Resonanz-Energie-Transfer erklärt. Für den hier zugrunde 
liegenden allosterischen Mechanismus, bei dem die Kraftverteilungsanalyse (FDA) verwendet wurde, konnte ein Signalnetzwerk, das von den PIP $_{2}$ - und ATP-Bindungsstellen bis zur Schnittstelle zwischen der FERM und der Kinasedomäne reicht, erstellt werden. Allerdings zeigen unsere Ergebnisse auch, dass die durch Liganden induzierten konformationellen Änderungen nicht ausreichend sind für eine vollstädige FAK-Aktivierung, was eine komplete Aufdeckung von Tyr576/577 erfordert. Dies legt nahe, dass ein zusätzlicher biochemischer oder mechanischer Stimulus zur Aktivierung der FAK notwendig ist.

Auf dises Grundlage erarbeiteten wir ein mechanisches Modell zur FAK-Aktivierung, in dem Zugkräfte, die sich von der Membran über die PIP 2 -Bindungsstelle der FERM Domäne und von der am Zytoskelett verankerten FAT-Domäne in FAK verbreiten. FAK können als Mechano-sensor fungieren, wenn diese Kräfte zu einer Aufdeckung zentralen Phosphorylierungsstellen der FAK (Tyr576/577) und damit zu einer Aktivierung von FAK führen. Um diese Hypothese von FAK als Kraftsensor zu testen, wurden molekular-dynamische Simulationen unter Krafteinwirkungen mit unterschiedlichen Ziehgeschwindigkeit, Ziehrichtungen und Membran-PIP 2 -Konzentrationen durchgeführt. Sie stützen direkt die Vermutung einer spezifischen kraftinduzierten Öffnung und Aktivierung der FAK. Dies ist bemerkenswert, da die kraftinduzierte Entfaltung der primär $\alpha$-Helikalen FERM und Kinasedomänen kompetitive Prozesse sind, aber es wurde beobachtet, dass sie im Vergleich zur Dissoziation der Domänen weniger bevorzugt werden. Um weitere Konsequenzen dises Mechanosensing zu ermitteln, wurde die kraftabhängige FAK-Kinetik, basierend auf extrapolierte MD-Daten, als Parameter zum Aufbau eines biochemischen Netzwerkmodells implementiert, welches die kraftabhängige FAK Aktivierung mit der nachgelagerten Austauschrate von GTP/GDP in Ras verbindet. Unsere rechnergestuitzte Studie liefert direkte Beweise für FAK-vermittelte mechanische Signalweiterleitung durch Ras zum Nukleus. Unsere Voraussagen können durch Experimente zur Zell-Dehnung und Einzelmolekülkraftspektroskopie direkt getestet werden. 


\section{CONTENTS}

$\begin{array}{lll}\text { Acknowledgements } & \text { v }\end{array}$

Abstract vii

Zusammenfassung $\quad$ ix

Contents $\quad$ xi

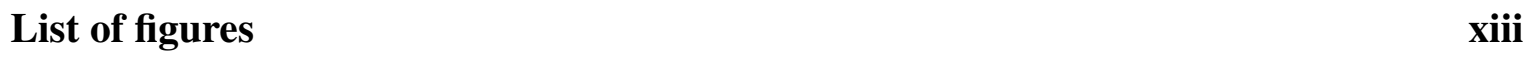

List of tables $\quad$ Xv

Abbreviation $\quad$ xvii

1 Introduction 1

1.1 Cell signaling and Focal Adhesion Kinase . . . . . . . . . . . . . . . 3

1.2 Atomic Force Microscopy . . . . . . . . . . . . . . . . . . . . . . 8

1.3 Dynamic force spectroscopy models . . . . . . . . . . . . . . . . . 9

1.4 Objectives and overview $\ldots \ldots \ldots \ldots \ldots$

2 Method and Theory 13

2.1 Molecular Dynamics simulation . . . . . . . . . . . . . 13

2.1 .1 Force field . . . . . . . . . . . . . . . . . . 16

2.1.2 Water model . . . . . . . . . . . . . . . . . . . . 19

2.1.3 Applying an external force . . . . . . . . . . . . . . 21

2.2 Molecular docking ........................ 23

2.3 Force distribution analysis $\ldots \ldots \ldots \ldots$. . . . . . . . . . . 25

2.4 Principal component analysis . . . . . . . . . . . . . . . . 27

2.5 Partial least squares functional mode analysis . . . . . . . . . . . . 28

2.6 Kinetic modeling . . . . . . . . . . . . . . . . . . . . . . . 29

3 Allosteric regulation of FAK by ligands binding 33 
3.1 Introduction . . . . . . . . . . . . . . . . . 33

3.2 Materials and Methods . . . . . . . . . . . . . . . 35

3.2.1 Molecular Docking . . . . . . . . . . . . . . . . . . 35

3.2.2 Molecular Dynamics simulations . . . . . . . . . . . . . . 36

3.3 ATP and PIP2-induced conformational changes of FAK . . . . . . . . . . 37

3.4 Detection of functional modes in FAK dynamics . . . . . . . . . . . . . . . 41

3.5 ATP and PIP2 induced allostery of FAK . . . . . . . . . . . . . . . 44

3.6 Discussion and Conclusions . . . . . . . . . . . . . . . . . . 51

4 Force-induced activation of Focal Adhesion Kinase 55

4.1 Introduction . . . . . . . . . . . . . . . . . . . . . 55

4.2 Materials and Methods . . . . . . . . . . . . . . . . 57

4.2 .1 FPMD simulations . . . . . . . . . . . . . . 57

4.2.2 PIP2 aggregation in POPE membrane bilayer . . . . . . . . . . 61

4.2.3 Fitting of rupture forces at varying loading rates . . . . . . . . . . 61

4.3 Force induced exposure of the activation loop. . . . . . . . . . . . . 63

4.4 PIP2 aggregation in POPE membrane bilayer . . . . . . . . . . . 66

4.5 FAK-membrane interactions . . . . . . . . . . . . . . . 67

4.6 Mechanism of force-induced FK-FAK opening . . . . . . . . . . . 71

4.6.1 Collective opening motion of FK-FAK . . . . . . . . . . . 71

4.6.2 Identifying force-carrying interactions at FERM F2- and kinase C-lobe 73

4.7 Force-dependent activation rates . . . . . . . . . . . . . . . . . . 79

4.8 Kinetic model of FAK signaling. . . . . . . . . . . . . . . . . . . . . . 83

4.9 Discussion and Conclusion . . . . . . . . . . . . . . . . . . . . 92

5 Conclusions and future perspectives $\quad 95$

$\begin{array}{ll}\text { References } & 101\end{array}$ 


\section{LIST OF FIGURES}

Figure $1.1 \quad$ A Scheme of ERK/MAPK signaling . . . . . . . . . . . . . 4

Figure 1.2 Domain organization and interactions of FAK . . . . . . . . . 5

Figure $1.3 \quad$ FERM basic patch and $\mathrm{PIP}_{2}$ lipid . . . . . . . . . . . . 7

Figure 1.4 Schematic representation of an AFM setup for force spectroscopy. . . 8

Figure 1.5 A schematic diagram of a single-well free energy profile . . . . . . 10

Figure $2.1 \quad$ Flow chart of a Molecular Dynamics procedure. . . . . . . . . . . . 14

Figure 2.2 Schematic diagram of the energy terms in force field. . . . . . . . . 17

Figure $2.3 \quad$ A scheme for the out-of-plane angle $\ldots \ldots \ldots \ldots$

Figure 2.4 Simple water models. . . . . . . . . . . . . . . . . 20

Figure 2.5 FPMD simulation setup of FAK . . . . . . . . . . . . 22

Figure 2.6 An example of a force profile. . . . . . . . . . . 23

Figure 2.7 The best scored FK-FAK-PIP 2 structure . . . . . . . . . . . . 23

Figure $2.8 \quad$ Energy profile of possible FK-FAK-PIP 2 structures. . . . . . . . . . . . 24

Figure $3.1 \quad$ Molecular structure of FAK FERM-kinase fragment. . . . . . . . . . 34

Figure 3.2 Grid-based molecular docking . . . . . . . . . . . . 35

Figure 3.3 Equilibrium simiulation box $\ldots \ldots \ldots \ldots$

Figure 3.4 Center-of-mass distance between F2/C-lobe. . . . . . . . . . . . . 37

Figure 3.5 Conformational changes of FAK upon ATP/PIP 2 binding measured by inter-domain distances. . . . . . . . . . . . . . . . . 38

Figure 3.6 The changes of RMSFs of FERM F2-lobe upon ATP and PIP $_{2}$ binding 39

Figure 3.7 RMSFs of the kinase domain upon ATP and $\mathrm{PIP}_{2}$ binding. . . . . . . 40

Figure $3.8 \quad$ Collective motions of the kinase domain . . . . . . . . . . . . . . 42

Figure 3.9 Conformational changes upon ATP/PIP 2 binding measured by PCA. . 43

Figure 3.10 Force distribution upon ATP binding. . . . . . . . . . . . . . . . . 45

Figure 3.11 Force distribution upon $\mathrm{PIP}_{2}$ binding. . . . . . . . . . . . . . 46

Figure 3.12 Force distribution upon ATP and $\mathrm{PIP}_{2}$ binding. . . . . . . . . . . . 47 
Figure 3.13 The force network connecting two ligands binding site to domain interface. . . . . . . . . . . . . . . . . . . 48

Figure 3.14 RMSFs of the activation loop upon ATP and $\mathrm{PIP}_{2}$ binding . . . . . . . 52

Figure $4.1 \quad$ Mechanical model of FAK activation. . . . . . . . . . . . . 56

Figure 4.2 Simulation system of FAK-membrane complex. . . . . . . . . . . . 60

Figure 4.3 An accumulative number of simulations at different steps during dissociation process. . . . . . . . . . . . . . 63

Figure $4.4 \quad$ Force-induced unfolding at C-terminus of the kinase domain. . . . . . 64

Figure $4.5 \quad$ Mechanism of the exposure of Tyr576/577 . . . . . . . . . . . . 65

Figure 4.6 Unfolding dissociation for different force applications . . . . . . . . . 65

Figure $4.7 \quad \mathrm{PIP}_{2}$ clustering in the POPE membrane. . . . . . . . . . . . . 66

Figure $4.8 \quad$ Mechanism of the exposure f . . . . . . . . . . . . . . . . . . 69

Figure 4.9 Contacts maps for all the FPMD simulations of FAK-membrane simulation. . . . . . . . . . . . . . . . 70

Figure 4.10 Partial least square functional mode analysis. . . . . . . . . . . . 72

Figure 4.11 Projection of force profiles on the area of F2/C-lobe interface. . . . . 73

Figure 4.12 Distribution of interfacial areas. . . . . . . . . . . . . . . . . . . 74

Figure 4.13 Change of F2/C-lobe interaction in time measured by TRFDA . . . . 76

Figure 4.14 Residue interactions at domain interface detected by TRFDA . . . . . 78

Figure 4.15 DHS fitting of rupture forces. . . . . . . . . . . . . . . . . . . . 79

Figure 4.16 Monte Carlo sampling of DHS model. . . . . . . . . . . . . . . . 80

Figure 4.17 Uncertainty in the parameters from DHS fitting. . . . . . . . . . . . 82

Figure 4.18 Multiple Grb2-mediated FAK signaling pathways. . . . . . . . . . . . 83

Figure 4.19 Scheme of two mechanisms of FAK auto-phosphorylation. . . . . . . . 84

Figure 4.20 Results of downstream FAK signaling. . . . . . . . . . . . . . . 85

Figure 4.21 FAK signaling pathway $\ldots \ldots \ldots \ldots$. . . . . . . . . 86 


\section{LIST OF TABLES}

Table $3.1 \quad$ List of residue pairwise forces in different protein states. . . . . . 50

Table 4.1 Structural properties of the membranes with different concentrations of $\mathrm{PIP}_{2}$ lipids. . . . . . . . . . . . . . . . . 67

Table $4.2 \quad$ Residue interactions at different domain opening steps. . . . . . . . . 77

Table 4.3 Kinetic parameters obtained from fitting the DHS model to the FPMD simulation data . . . . . . . . . . . . . . . . . . . 81

Table $4.4 \quad$ List of parameters for FAK signaling model. . . . . . . . . . . . . 87

Table $4.5 \quad$ List of Reactions in FAK signaling model. . . . . . . . . . . . . . . 89

Table $4.6 \quad$ List of species in FAK signaling model. . . . . . . . . . . . . . 91 



\title{
ABBREVIATION
}

\author{
AFM . . . A Atomic force microscopy \\ apo-FK . . . F FERM and kinase domain fragment \\ COM .... . Center of Mass \\ DHS . . . . . . Dudko, Hummer and Szabo \\ ECM .... Extracellular matrix \\ ERK . . . . . . Extracellular-signal-regulated Kinase \\ F-FAK . . . . FAK FERM domain \\ FAs . . . . . Focal adhesions \\ FAK . . . . . . Focal Adhesion Kinase \\ FDA . . . . . Force Distribution Analysis
}

FK-ATP . . . . ATP bound FK-FAK

FK-ATP-PIP 2 . FK-FAK bound to ATP and PIP 2

FK-FAK . . . . Crystal structure of FAK FERM and kinase domain fragment

FPMD . . . . Force Probe Molecular Dynamics

FRET . . . . F Fluorescence Resonance Energy Transfer

Grb2 . . . . G Growth factor receptor-bound protein 2

K-FAK . . . . FKA Kinase domain

MAPK . . . . Mitogen-activated protein kinase

MD . . . . . Molecular Dynamics

ODE . . . . . Ordinary differential Equation

OPLS . . . . O Optimized Potential for Liquid Simulations 
PCA . . . . . Principal Component Analysis

PME . . . . Particle Mesh Ewald

PIP $_{2}$. . . . . Phosphoinositide phosphatidylinsositol-4,5-bisphosphate

PIP5KI $\gamma$. . Phosphatidylinsositol 4-phosphate 5-kinase type I $\gamma$

PLS-FMA . . . Partial Least Square functional Mode Analysis

POPE ... . 1-Palmitoyl-2-oleoyl-sn-glycero-3-phosphoethanolamine

REDS . . . . RESP charge Derive Server

RMSD . . . . . Root Mean Square Deviation

RTK . . . . . Receptor Tyrosine Kinase

SH2 . . . . . S Src homology 2

SOS . . . . . Son of Sevenless

Shc ..... S Src SH2 domain-containing proteins

TRFDA . . . . Time-resolved Force Distribution Analysis 


\section{CHAPTER 1}

\section{INTRODUCTION}

In complex biological systems, cells, which are often called the "building blocks of life", rarely live in isolation. Most of the cells are required to receive and process a specific combination of signals from the external environment and from neighboring cells to survive. Different types of cells require a different combination of signals, which makes it specific to different compartments of the body. To form and maintain different types of tissues, multiple cell types in varying numbers, morphology and localization are required to coordinate with one another in order to perform the required task. Even in unicellular organisms, cells work as non-isolated components to accomplish tasks that could not otherwise be carried out by a single cell. Therefore the ability of cells to respond to environmental changes is an essential feature of life.

Cells can communicate with one another over short or long distances, or even through direct contact, by following several signal transmission strategies mediated by different networks such as metabolic or inter-cell signaling. Signals of chemical, electrical and mechanical nature are generated during intercellular communications. Once a signal reaches the surface of the target cell, it triggers multiple intracellular signaling pathways through cell surface receptors. Errors in cell communication pathways can lead to cell malfunction or even death, causing major human diseases, such as cancer, autoimmunity and diabetes.

An increasing body of evidence suggests that cells are very sensitive to mechanical signals. Mechanical signaling within a tissue is crucial for tensional homeostasis between and within cells, which modulate cellular processes, such as differentiation, development, 
and survival $[1,2]$. For example, bone renewal occurs only upon physical exercise and gravity forces, since mesenchymal stem cells require mechanical forces to differentiate [3]. The response to mechanical perturbations often insinuates protein allostery [4]. External mechanical force can induce deformation of cell membrane and surface receptors, such as integrin, leading to structural changes in focal adhesions (FAs). FAs are enormous multimolecular complexes located at the periphery of the cell and comprise a number of mechanoresponsive proteins such as integrin [5], talin [6-9], vinculin [10, 11] and p130Cas [12, 13]. Through FAs, mechanical signals from the extracellular matrix (ECM) are able to activate the cytoskeleton in the cell [14] and consequently regulate gene expression and cell growth. Moreover, they can also induce the re-modeling of the cytoskeleton by way of shear flow and activation of stretch-activated ionic channels, resulting in a change of $\mathrm{pH}$ value or transport of small molecules. The crucial role of mechanical signaling is broadly known. However, the mechanisms underlying the conversion of the external forces responsible for mechanical signaling to intracellular chemical signals still often has remained unclear.

Mechanosensors are key regulators in the transduction of mechanical signals. As distinct from the ligand binding-induced allosteric activation following the lock and key model, force sensors undergo controllable conformational changes in response to an external force. The function of a mechanosensor refers to the propagation and amplification of the physical forces from the surface to the inside of the cell and to the translation of the signals into several cellular processes. Thus, studies of the force-induced deformation of mechanosensory proteins are straightforwardly related to the mechanisms of mechanical signaling in cells.

Until now, a set of techniques has been developed to perform force spectroscopy in order to study the mechanical response of biomolecules to stretching or torsional forces. These techniques include atomic force microscopy (AFM) [15], optical and magnetic tweezers [16] [17], microneedles [18], and biomembranes [19]. They can be used to measure the mechanical properties of a single protein, membrane or even an individual chemical bond, providing the details in relation to the conformational changes, unfolding forces, stability and activity.

The rest of this chapter will briefly introduce the role of a mechanosensory protein, Focal Adhesion Kinase (FAK), in cell signaling and then will present the AFM method, which is one of the favored ways to study force induced protein activation and unfolding. As most mechanosensors, the activation and function of FAK depend on its force-induced conformational changes. The protein response to force measured in AFM or other single 
molecule stretching experiments or observed in computer simulations will depend on the way of force application. In short, the higher the applied forces, the faster the induced conformational transition. This relationship has been addressed by a series of theoretical models, that will be introduced in Section 1.3. Finally, Section 1.4 introduces the specific objectives of this work and gives an overview of this thesis.

\subsection{Cell signaling and Focal Adhesion Kinase}

Signal transduction from the cell surface to the nucleus is achieved by multiple signaling pathways, one well-studied example being the Mitogen-activated protein kinase (MAPK) pathway. Protein phosphorylation plays a key regulatory role in such signaling pathways by switching on (by phosphorylation) and switching off (by dephosphorylation) the activation of signaling proteins. This reversible and dynamic process is mediated by the following reactions [20]:

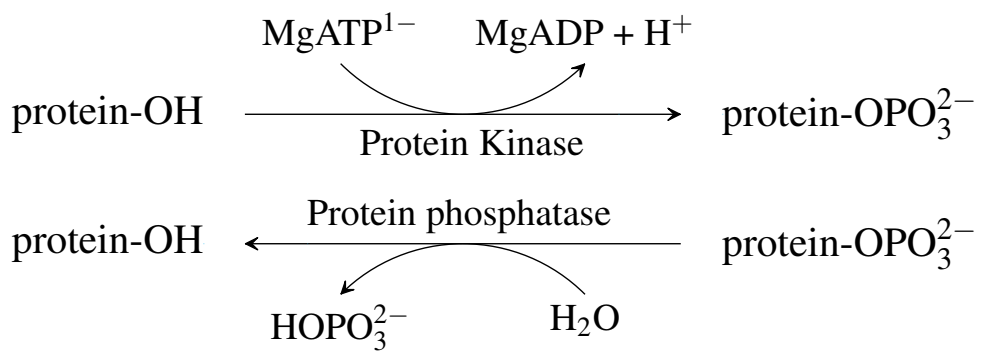

The protein phosphorylation sites in provide reorganization sites for downstream proteins. Protein kinases are thus the essential molecular switches of various biological functions. These enzymes are classified as serine/threonine or tyrosine kinases depending on the -OH group they phosphorylate. To date, 90 protein tyrosine kinases are identified in the human genome. 58 of them are receptor and 32 are non-receptor proteins [21].

One of the best-known signaling pathways, the extracellular-signal-regulated kinase (ERK) pathway, which is one of the major signaling cascades of the MAPK signaling pathway [22] (Figure. 1.1), begins with the binding of a ligand, e.g. Epidermal growth factor (EGF), to the extracellular domain of a receptor tyrosine kinase (RTK). Most RTKs are multimeric complexes. Each monomer consists of an extracellular ligand binding domain (N-terminus), a single hydrophobic transmembrane helix, and an intracellular C-terminal domain containing multiple tyrosine sites. Initially, the monomers of a RTK are located se- 


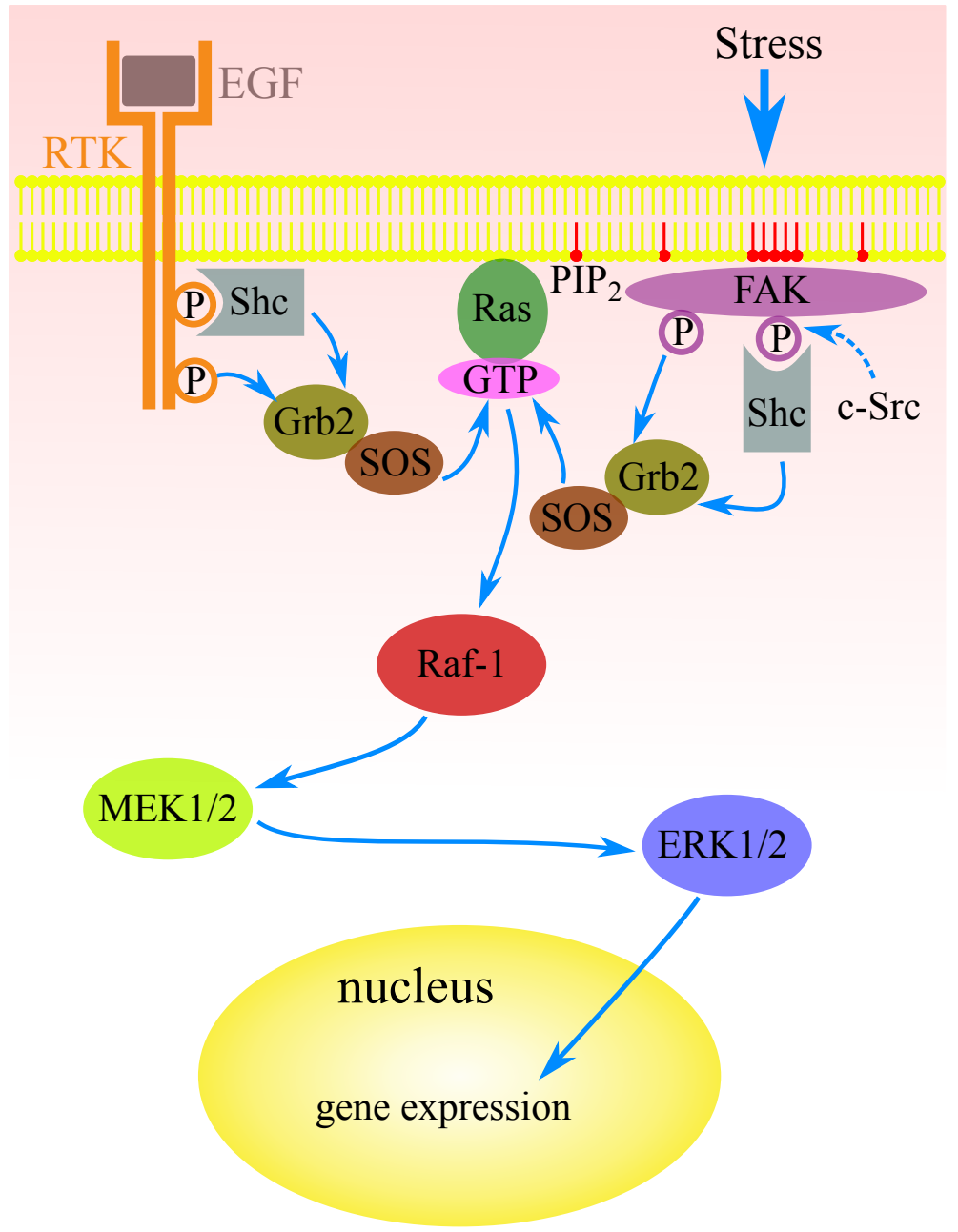

Figure 1.1 A scheme of the ERK/MAPK signaling pathway. Chemical signals such as EGF bind to RTK in the cell membrane and induce autophosphorylation in the cytoplasmic domain of RTK. Then, through a number of sequential phosphorylations of downstream proteins, the signal is transduced into the cell nucleus. Mechanical signals act on the cell membrane and trigger the activation of a non-receptor tyrosine kinase, FAK, which is located at the cytoplasmic face of the membrane bilayer and mediates the signal transduction into the cell nucleus through a pathway constituted by a chain of similar interactions.

parately from each other. Ligand binding leads to the dimerization of two subunits of the RTK. Then, the RTK dimer auto-catalyzes the phosphorylation of its tyrosine sites in the intracellular domain, which provides the binding site for the $\mathrm{SH} 2$ domain of the growth factor receptor-bound protein 2 (Grb2). Next, the two SH3 domains of Grb2 bind to the GDP/GTP exchange factor, Son of Sevenless (SOS) and induce its activation. The activated SOS recruits the membrane bound protein Ras and promotes the exchange of Ras bound GDP against GTP. This exchange induces the activation of Ras and enables the bind- 
ing of Ras with Raf kinase. Subsequently, the activated Raf kinase phosphorylates and activates Mitogen-activated protein kinases (MEK1 and MEK2), which eventually leads to the phosphorylation and translocation of ERK. Once in the nucleus, ERK activates several transcription factors and mediates gene expression. The malfunction of the ERK/MAPK pathway leads to uncontrolled cell proliferation and increasing cell resistance to apoptosis, which causes tumor.

In contrast to chemical signaling transduction via receptor tyrosine kinases, mechanical signal transduction is suggested to be mediated by a non-receptor tyrosine kinase, Focal Adhesion kinase, FAK, in the ERK/MAPK signaling pathway. As most non-receptor tyrosine kinases, FAK is localized in the cytoplasm, lacking the extracellular ligand binding domain and the transmembrane region. FAK comprises a central tyrosine kinase domain (orange) flanked by two large non-catalytic domains (Figure. 1.2). The kinase N-lobe follows a 50-residue linker, through which it is connected to the N-terminal FERM (4.1 ezrin, radixin, moesin homology) domain containing 3 lobes (F1-F3, blue). The kinase C-lobe is connected to the C-terminal FAT (focal adhesion targeting, violet) domain through a 220 residues long proline-rich and disordered region (yellow).

a)

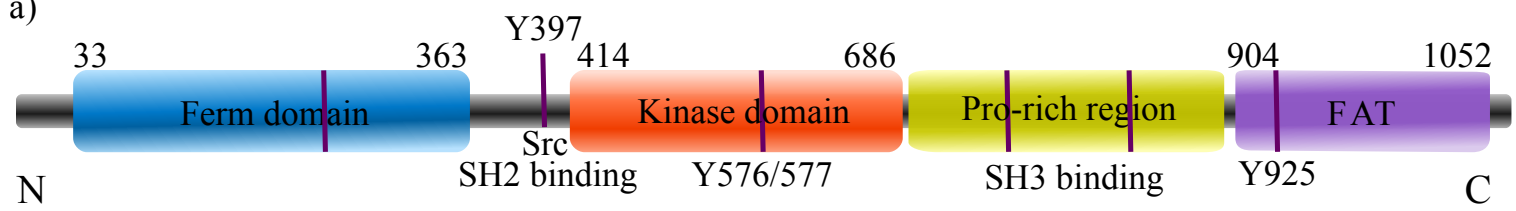

b)

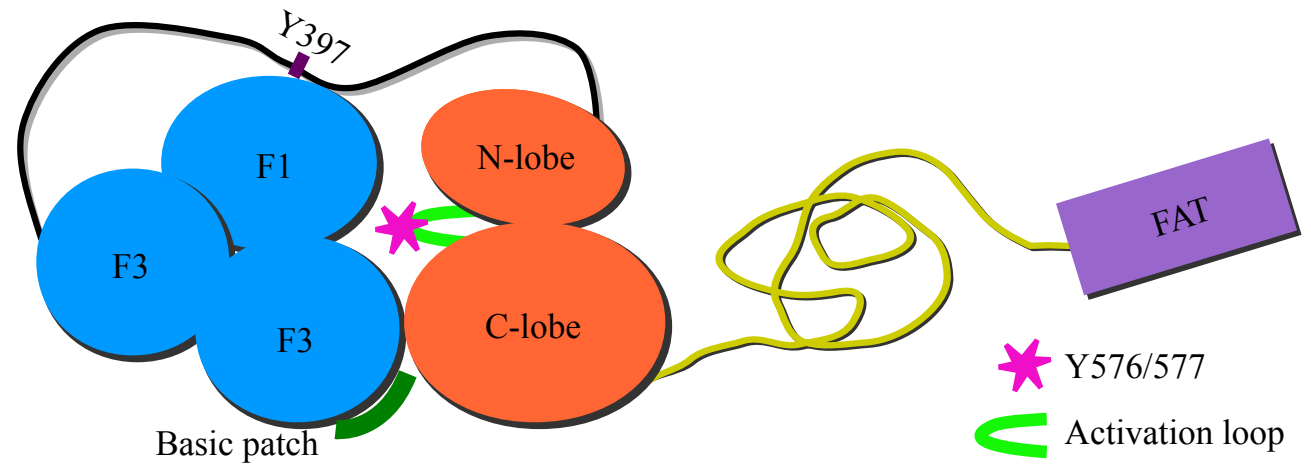

Figure 1.2 FAK organization. (a) Domain organization of FAK including phosphorylation sites. (b) Schematic representation of FAK domains. The colors correspond to the ones used in (a). The organization of F1-F3 lobes in the FERM, N and C-lobes in the kinase domain is shown. The basic patch (dark green), the phosphorylation site of Tyr576/577 (purple star) and the activation loop (light green) are highlighted.

Upon cell adhesion to the extracellular matrix, FAK is translocated towards focal adhesion sites and phosphorylated on the cytosolic face of the plasma membrane [23-25]. 
Through adhesive interactions with other focal adhesion components, including Src, CAS, and paxillin, FAK regulates the formation and function of FAs [26, 27]. Acting as a signaling hub between integrin and multiple downstream signaling pathways, FAK carries out diverse functions in embryonic development and survival, and is involved in cancer progression and cardiovascular diseases [28-30]. Studies have also shown that it mediates force-guided cell migration [31] as well as strain-induced proliferation [32]. Recently, the mechano-sensitivity of FAK has been ascribed to the force-sensing fibronectin-integrin link [33]. However, the available data on mechano-sensing through FAK are indirect to date, and it remains unknown if FAK lies downstream of mechano-sensing processes such as those involving integrins, or if FAK also per se is exposed to and activated by mechanical force.

As reported by previous studies, the activation of FAK starts with the autophosphorylation of Tyr397 in the linker between the FERM and kinase domains [34]. This provides a high-affinity binding site for the Src homology 2 (SH2) domains of the Src family kinase such as c-Src and Src SH2 domain-containing proteins (SHC) and results in activation of FAK. The following phosphorylations of Tyr576 and Tyr577, which are located in the activation loop of FAK, induce the maximum activity of the kinase domain [35]. However, the crystal structure of FK-FAK [36], a fragment comprising the FERM and kinase domains, suggests an auto-inhibitory conformation of FAK by an intra-molecular interaction between the FERM and kinase domains. Auto-inhibition is mediated through the burying of the phosphorylation sites represented by Tyr576 and Tyr577 (purple) at the binding interface between the two domains as also shown by the scheme in Figure. 1.2b. Thus, the release of Tyr576/577 from the FERM domain is the major regulatory mechanism of FAK activation. Although, the mutations Y180A and M183A at the FERM F2-/kinase C-lobe have been proved to result in a complete opening of FK-FAK and significantly increased FAK activity [36], the mechanism of FAK catalytic turnover, through the release of Tyr576/577 from FERM domain, still remains elusive to date.

To reveal the mechanism of FAK catalytic turnover by exposing the phosphorylation site Tyr576/577, many stimuli have been studied, such as integrin signaling [37] and growth factor receptors [38-40], but molecular details of these processes are still unclear. For the last decade, a key role of phosphoinositide phos-phatidylinsositol-4,5-bis-phosphate ( $\left.\mathrm{PIP}_{2}\right)$ in these interactions has been emphasized [41, 42]. As a ubiquitous second messenger, $\mathrm{PIP}_{2}$ modulates divers biological processes, including signaling, intracellular traffic, and cellular motility [43]. $\mathrm{PIP}_{2}$ is enriched in the inner leaflet of plasma membrane and can be 
a)

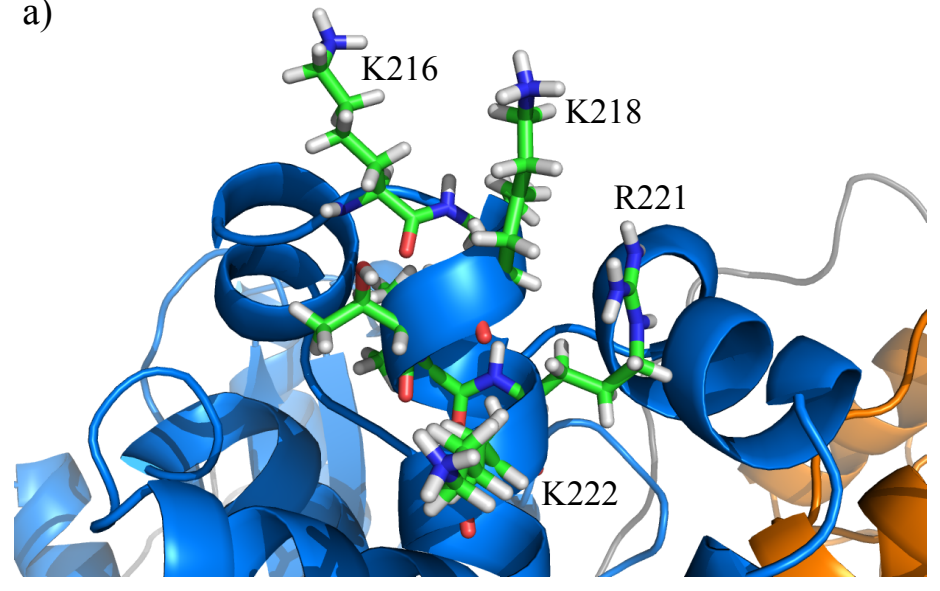

b)

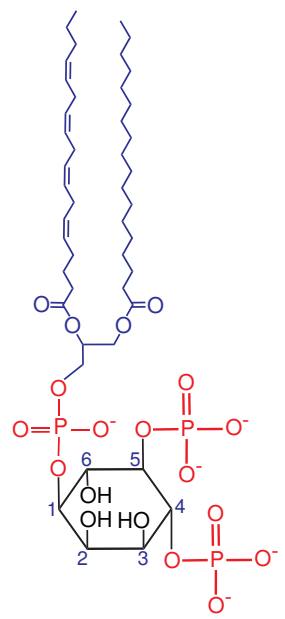

Figure 1.3 FAK PIP 2 binding. (a) Positively charge basic patch, ${ }^{216} \mathrm{KAKTLR}^{221}$ (shown as sticks), which is located in the FERM F2 lobe (shown as blue cartoon). (b) Molecular structure of the phosphatidylinositol 4,5-bisphosphate ( $\left.\mathrm{PIP}_{2}\right)$ lipid.

locally produced in FAs by the enzyme phosphatidylinsositol 4-phosphate 5-kinase type I $\gamma$ (PIP5KI $\gamma$ ), which adds the 5-phosphate group to PI(4)P. The experimental evidence shows that PIP5KI $\gamma$ is required for efficient FAK activation, providing strong support to the notion that $\mathrm{PIP}_{2}$ plays important role in the mechanism of FAK signaling [41]. It was also demonstrated that the direct interaction of highly negatively charged $\mathrm{PIP}_{2}$ and the positively charged basic patch $\left({ }^{216}\right.$ KAKTLR $\left.^{221}\right)$ (see Figure. 1.3$)$ in the FERM domain [41, 42] leads to the partial activation of FAK in vitro [42] and in vivo [44]. Thus, the insight of molecular description of FAK allosteric regulation by PIP $_{2}$ can be helpful to reveal the mode of FAK activation.

Once Tyr576 and Tyr577 are exposed to the solvent, they are phosphorylated by Tyr397 bound c-Src and provide second binding site for it. The FAK-Src complex triggers the further phosphorylation of Tyr925 in FAK FAT domain. Immediately after, the phosphorylated Tyr925 binds to Grb2 [45], so that FAK contributes to the regulation of Ras GTP/GDP exchange and other downstream signaling pathways, including the Raf kinase and MAPK (Figure. 1.1). Therefore, understanding the activation mechanism of FAK is crucial for the study of mechanical signal transduction from the ECM to the cell nucleus. To test the hypothesis of FAK as a force-sensor, a set of computational studies, have been undertaken in this work. The allosteric effect of $\mathrm{PIP}_{2}$ on the FAK conformation is investigated in equilibrium (Chapter 3) and under external force (Chapter 4), which is suggested to act as an additional stimulus for FAK activation. 


\subsection{Atomic Force Microscopy}

Atomic force microscopy (AFM) is a novel technique, which has been initially conceived as high-resolution scanning probe microscopy to investigate the morphology and structure of electrical solid material surfaces. Nowadays, it also has applications in biology to study the topological features and mechanical properties of nonconductive surfaces, such as crystal structures of proteins, cell membranes and membrane proteins. The setup of an AFM comprises of 4 parts: a hard tip mounted at the end of a flexible cantilever, a laser beam, a photo detector and a computer-controlled piezoceramics stage (Figure. 1.4). An AFM scans the surface of samples through the tip. The interaction between the AFM tip and the sample causes the bending of the cantilever that is detected by a laser beam reflected from the upper side of the cantilever, and eventually recorded by the photo detector.

Most of the AFM tips are made of silicon or silicon nitride and have a rounded shape with a radius in the range of 5-50 nanometers $(\mathrm{nm})$. The interaction area between the AFM tip and the sample is dependent on the size of the tip. An extremely small AFM tip allows measurements at atomic resolutions. The force sensitivity is dependent on the stiffness of the cantilever and the power of the laser. The resolution of forces detected by a very soft spring can reach into the pico-Newton $(\mathrm{pN})$ range.

The sample is positioned by a computer-controlled piezoceramics stage. The cantilever can scan the sample in two ways. The AFM tip scans the surface in the $\mathrm{x}-\mathrm{y}$ direction so 
that a three-dimensional topological image of the sample can be created, or alternatively, the technique is used to do force spectroscopy (Figure 1.4). In this case, the AFM tip is moved along the z-direction, i.e., first moved towards the sample until they are in contact with each other, then retracted from the sample until no interaction between them is felt. In this process, the interactions between sample and AFM tip can be measured in terms of force, resulting a position-dependent force profile. The resulting forces are obtained from the cantilever deflections by using Hook's law. As the AFM tip gets close to the sample, the interactions between them, which cause the cantilever to bend towards the sample, are attractive forces, including van der Waals and electrostatic forces. In contrast, repulsive forces (electrostatic) are detected when the cantilever bends away from the sample. At each distance, when the measured forces equal the interactions between AFM tip and sample, the system is in equilibrium. The faster the sample is pulled, the further the system is away from equilibrium.

AFM based force spectroscopy can be carried out in two modes. In the first mode, the force clamp mode, a constant tensile force is applied to the sample by adjusting the position of the cantilever. In the second mode, defined as the force extension mode, the sample is stretched by the AFM tip at constant speed and the variations in force are recorded. In this work, force probe Molecular Dynamic (FPMD) simulations (introduced in Section 2.1.3) have been performed to mimic the force extension mode of an AFM experiment (Chapter 4). This study provides insights at a molecular level of protein unfolding and hence helps revealing the underlying mechanism of FAK mechanical activation.

\subsection{Dynamic force spectroscopy models}

The function of a protein is always related to its three-dimensional conformation. In the previous studies, many proteins have been reported to be activated or regulated by an external force, such as Von Willebrand factor [46] in blood vessels, titin kinase in muscles [47], vinculin [11] and FAK [48] at FAs. To study the functions of such mechano-sensing proteins, it is important to understand the role of force in these biological processes.

Many force-regulated processes involve protein sequential unfolding or multi-domain dissociation, i.e., the external force regulates the transition between folded and unfolded state of a protein or the transition between the opened or closed protein conformation. With 
regard to biochemical reactions, these processes can be described as first-order reactions: [state $\mathrm{A}(\lambda=0) \rightarrow$ state $\mathrm{B}(\lambda=1)$ ] (Figure. 1.5). The reaction rate, $k_{0}$, can be expressed by Arrhenius' law:

$$
k_{0}=A e^{-\Delta G^{\ddagger} / k_{B} T}
$$

where $\mathrm{A}$ is the pre-exponential factor, which is reaction-specific, $\Delta \mathrm{G}^{\ddagger}$ represents the height of the free energy barrier, $k_{B}$ is the Boltzmann constant, and $\mathrm{T}$ is the temperature. This equation describes a temperature-dependent process, but what is the influence of force in this process? To answer this question, Bell suggested a model that directly includes force. As can be seen in Figure. 1.5, an external constant force $F$ functions as a catalyst in the transition between states and reduces the height of the free energy barrier $\Delta G^{\ddagger}(F)$, thereby accelerating the reaction rate $k_{F}$. The work performed by force is expressed as $\mathrm{F} x_{b}$, where $x_{b}$ is the distance along the reaction coordinate between state $\mathrm{A}$ and the transition state and is equal to the reduced energy barrier $\left(\Delta \mathrm{G}^{\ddagger}-\Delta \mathrm{G}^{\ddagger}(\mathrm{F})\right)$. Thus, the force-dependent reaction rate, $k_{F}$, can be expressed as:

$$
k_{F}=A e^{-\left(\Delta G^{\ddagger}-F x_{b}\right) / k_{B} T}=k_{0} e^{F x_{b} / k_{B} T}
$$
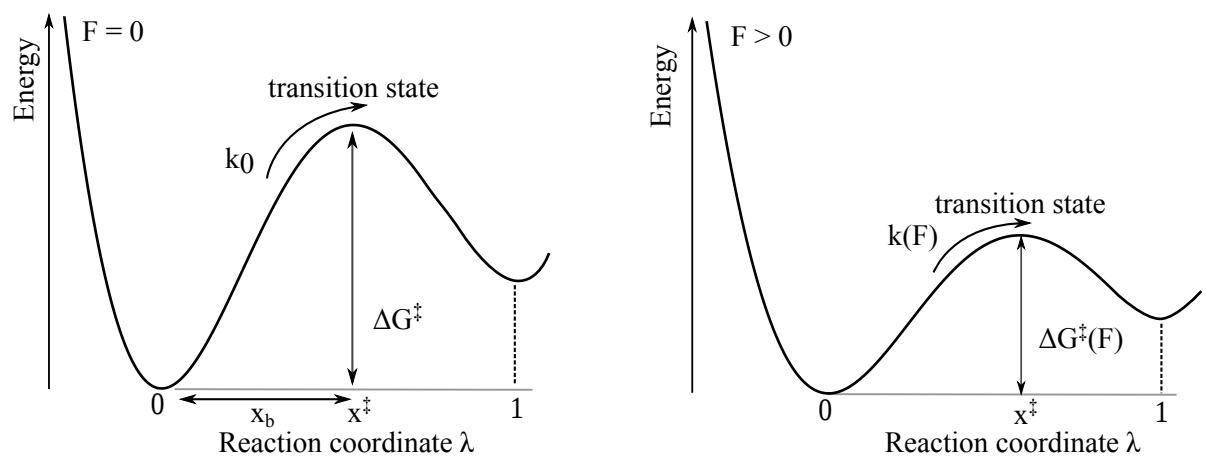

Figure 1.5 A schematic diagram of the free energy surface for a transition between state $A(\lambda=0)$ and state B $(\lambda=1)$ with (right) and without (left) an external force. $x_{b}$ is the distance between state A and the transition state at $x^{\ddagger} . \Delta G^{\ddagger}$ and $k_{0}$ are the free energy barrier and reaction rate of the transition without force. $\Delta G^{\ddagger}(\mathrm{F})$ and $k_{F}$ are the force-dependent free energy barrier and reaction rate.

The Bell model is often used to analyze the kinetic data obtained form force spectroscopy experiments as well as from MD simulations. However, comparing the analysis of experiments and simulations using the Bell model is limited due to the fact that the ranges of applied forces and velocities between computer simulations and experiments are largely different, while the approximations of the Bell model limits its applicability to a wide range of forces or velocities. Firstly, the model assumes that the transition along the reaction co- 
ordinate is exclusively affected by a force-dependent process corresponding to a single-well free energy landscape. Secondly, it assumes that the force-dependent reaction rate, $k_{F}$, is described by an exponential law, which can be thought as an oversimplification. Finally, the Bell model neglects the effect of force on the distance between the reactant and transition state, i.e., the energy barrier is only lowered by the external force, but not shifted. The study by Li et al. [49] have shown that the pulling force indeed shifts the transition state.

To reduce the assumption limit of Bell model, Dudko, Hummer and Szabo (DHS) introduced a stochastic model [50] of a spring and Kramers' theory [51] of diffusion to estimate the kinetic parameters. The DHS model is expressed as:

$$
k_{F}=k_{0} \alpha^{(1 / v)-1} e^{\Delta G^{\ddagger} /\left(k_{B} T\right)\left(1-\alpha^{1 / v}\right)}
$$

where $\alpha=1-v F \Delta x / \Delta G^{\ddagger}$ and $v$ is a approximated factor, which depends on different nature of underlying free energy landscape. $v=2 / 3$ and $v=1 / 2$ correspond to a linear-cubic and a cusp free energy surface, respectively. For $v=1$, the DHS model is the same of the Bell model. For $v \neq 1$, the Bell expression becomes invalid, when $\mathrm{F}<\Delta \mathrm{G} /\left(v x^{\ddagger}\right)$.

In this work, both models will be tested and applied to analyze the kinetic data derived by our MD simulations to investigate the force-dependency of FAK activation (for more details see Chapter 3 and 4). The estimated activation rate will be further used as kinetic parameter to establish a mathematic model of FAK signaling (Chapter 4).

\subsection{Objectives and overview}

In this work, we attempt to understand the role of FAK in the regulation of signal transduction from the cell periphery to downstream proteins, by converting the mechanical signals into biochemical reactions. We addressed two major questions: First, how is FAK regulated biochemically, i.e. by ligand binding, as observed by recent FRET measurements? Second, how are FAK and downstream signaling activated mechanically? We performed MD simulations and biochemical network simulations to reveal the mechanisms of underlying FAK activation, which is inaccessible by biological experiments.

In Chapter 2, we briefly explain the simulation techniques and analysis methods used throughout this study. This includes molecular docking and Molecular Dynamics (MD) 
simulations to predict effects of ligand binding on FAK dynamics and function. Section 2.2.3 explains how to apply an external force during MD simulations, which is a central method of this study. This allowed us to examine the force-induced activation of FAK under various conditions. Analysis of simulated trajectories was based, among others, on Principal component analysis (PCA), Partial least square functional mode analysis (PLS-FMA) and Force Distribution Analysis (FDA).

Chapter 3 presents results on ligand-induced conformational changes of FAK. The computational results are consistent with experiment results from previous studies [41]. PCA here was used to detect the global motions in FAK dynamics and to interpret the experimental result on a molecular level. Next, FDA was carried out to reveal how the signal is transduced from the distant ligand binding site to the domain interface within the FAK protein structure. The results shown in this Chapter suggest that the allosteric effect of ligand binding is not sufficient to induce FAK catalytic turnover. External force as an additional stimulus might enhance FAK activation.

Following the conclusions of Chapter 3, we examined if FAK activation can be induced by a mechanical stretching force. To this end, we applied an external force to FAK in MD simulations. The way of force application is meant to mimic as close a possible force spectroscopy experiments of the FAK FERM-kinase domain dissociation as required for FAK maximum activation (Figure. 1.4). Thus, the force induced FAK turnover is the subject of Chapter 4. Here, extensive control simulations with varying loading rates, pulling directions and membrane $\mathrm{PIP}_{2}$ concentrations were carried out. To characterize the domain dissociation process, the interactions between FERM F2 and Kinase C lobe were calculated in form of punctual stress (Section 2.3) along the pulling direction by using time-resolved force distribution analysis (TRFDA). We then estimated the kinetic parameters, including the reaction rate as a function of force and, the width and height of the free energy barrier, from the Bell and DHS models (Section. 1.3). The resulting force-dependent reaction rate serves as a parameter in a mechano-biochemical network model (section 2.6) to connect force dependent FAK kinetics to the downstream RasGTP concentrations.

At last, we summarize the major conclusions of this thesis in Chapter 5. We also present some of the limitations and challenges that we met during this work and propose an outlook on further studies on mechanical signal transduction. 


\section{CHAPTER 2}

\section{METHOD AND THEORY}

\subsection{Molecular Dynamics simulation}

Molecular dynamics (MD) simulation is a computational method of calculating the time dependent behavior of complex systems and providing detailed information about the structure, dynamics and thermodynamics of biomolecules and their complexes. This method is based on well-established classic physical laws. MD simulations, acting as a linker, connect theoretical and experimental studies in two ways: on one hand, they can provide an interpretation of experimental observations at atomic scales, and on the other hand, they are able to predict new mechanisms of biochemical processes, which are required to be validated by experiments. Furthermore, MD simulations can also simulate systems, which are difficult or impossible to study in the laboratory due to limitations of the experimental techniques.

Theoretically, the dynamics of any molecule can be completely described by solving the time-dependent Schrödinger equation, which is given as

$$
\mathscr{H} \Psi=E \Psi
$$

where $\mathscr{H}$ is the Hamiltonian operator. When a certain wavefunction is operated upon by $\mathscr{H}$, it returns the same wavefunction multiplied by the energy $E$ of the state. The Hamiltonian is expressed in terms of electron-electron repulsion, electron and nuclei attraction, and nuclei and nuclear repulsion. Solving this function numerically gives the wave func- 
tion of each electron. However, in practice, the computational resources limit the usage of such quantum mechanics (QM) calculations and numerical approximations to less than 100 atoms. Since a molecular system of a single protein solved in a water box comprise usually more than 10,000 atoms, several reasonable approximations are made to describe such large molecular systems at the molecular mechanics (MM) level.

Most importantly, the Born-Oppenheimer approximation assumes that electrons immediately follow the motion of nuclei, because they have much less weight and move much faster. By following this approximation, the atoms and the bonds are represented as balls and springs, respectively, in a model of protein. Moreover, electrons are not directly considered in the simulation, but their implicitly included in the parameters, which are assigned to different atom types. The interactions between atoms are approximated by using empirical functions, which are termed as force field (see 2.1.1). The last key approximation is that the dynamics of the atoms are described by classical mechanics.

A Molecular Dynamics procedure can be briefly summarized in the form of a flow chart as shown in Fig. 2.1. For an MD simulation of a single protein solved in water, the initial state is defined by the positions, velocities and forces of all the atoms present in the system. The structure of the protein is obtained by X-ray crystallography, Nuclear Magnetic Resonance (NMR) or computational homology modeling. The initial velocities of the atoms are generated from random numbers and the initial forces acting on the atoms are calculated by using a force field (Section 2.1.1). Once all this information is known, the dynamics of the atoms are derived by integrating Newton's equation of motions with respect to time:

$$
F_{i}=m_{i} a_{i}=m_{i} \frac{d v_{i}}{d t}=m_{i} \frac{d^{2} r_{i}}{d t^{2}}
$$

where the total force $F_{i}$ acting on atom $i$ is equal to the product

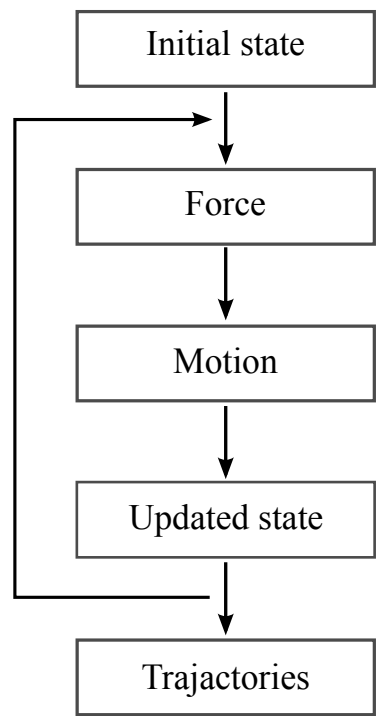

Figure 2.1 Flow chart of a Molecular Dynamics procedure. of its mass $m_{i}$ and its acceleration $a_{i}$, and can also be expressed in terms of the velocity $v_{i}$ of the coordinates $r_{i}$. The integration of atomic motions can be achieved by using numerical algorithms such as verlet and leapfrog methods. They provide new coordinates and velocities of all atoms, which are used to start a new cycle of MD simulation. All the micro-states calculated by an MD simulation are collected in 
a trajectory. The integration time-step corresponding to the time scale of a cycle of MD simulation is chosen by regarding the time scale of the fastest degree of freedom in the simulation system, e.g. the femtosecond scale chemical bond vibrations in a protein system. In practice, the very high vibrational frequencies are not of interest in classical computer simulations. Instead, the intra-molecular bonds are restricted to a fixed length by using constraint algorithms such as SHAKE [52] or LINCS [53] so that the integration time-step can be increased to 2-4 fs. In this work, we use a 2 fs integration time-step in all simulations.

In vitro or in vivo, a single biomolecule of interest can be studied in a certain environment, e.g. surrounded by solvent or in presence of membrane bilayer. Because of the limited computational resources, only a system with a finite size can be simulated. i.e. an MD simulation system can only have a size at microscopic length scale in contrast to the macroscopic experimental setup. To remove the artifacts caused by a simple boundary assigned to the simulation box, MD simulation often use period boundary conditions (PBC), allowing a simulation system as shown in Fig. 3.3 to be infinitely replicated at each side of the box. When an atom moves beyond one side of the box, it reappears at the opposite side of the box. It has to be noted that the size of the simulation box has to be chosen large enough to prevent the molecule of interest to interact with its periodic image from a virtual neighbor box.

For a system comprised of $N$ atoms, each atom is supposed to interact with all other atoms in the system through non-bonded interaction, e.g. interactions of an atom in a protein with water molecules. As a consequence, the number of non-bonded interactions scales with $N^{2}$, thus, the evaluation of these non-bonded interactions requires most of the computational expense of the force calculation at each integration step. Since the non-bonded interaction is described as a function of the inverse of the inter-atomic distances in the force field (Section 2.1.1), the interaction energy decreases with the increasing in distance, i.e. the non-bonded interaction of an atom with another atom beyond a certain cut-off distance is negligible. By introducing this cut-off distance into the calculation of forces, the number of interactions can be decreased 2-3 orders of magnitude, which results in a significant increase in computational efficiency. However, as the Coulomb potential (in terms of $r^{-1}$ ) decreases much slower with increasing distance than the Lennard-Jones potential (in terms of $r^{-6}$ and $r^{-12}$ ), the cut-off treatment causes artifacts by ignoring the electrostatic interactions induced by the interactions with the atoms beyond this cut-off distance. To recover this contribution to the total potential energy, Particle Mesh Ewald (PME) is used. Thus, the electrostatic interactions are divided into two parts. The first part (within the short range cut-off) is cal- 
culated in real-space. The second part (so-called long-range electrostatic interactions) is obtained using PME sums, i.e. from interactions of charges on a grid estimated by using Fourier transforms. The calculations of these short range and long range interactions can provide an accurate estimation of the total non-bonded potential energy in the system with reasonable computing speed.

MD simulations can be performed such that they conserve different properties of the whole system, including the number $N$ of particles, temperature $T$, pressure $p$, energy $E$ and volume $V$. Some of these properties are usually kept constant simultaneously. All micro-states, which are generated by the MD simulations with the same setup, constitute an ensemble. In an ideal $N V E$ MD simulation, which has a constant total energy $E$, number $N$ of particles and volume $V$, the time averages computed along the trajectory are the same as the ensemble averages in the microcanonical ensemble. However, in practice, simulations show an energy difference due to the cut-off, numerical in occurrence, etc. The easier way is to perform an NVT simulation (canonical ensemble) that uses an average temperature controlled by a thermostat algorithm instead of a conserved energy. In an $N p T$ ensemble (isothermic-isobaric ensemble), in addition to control the temperature, the pressure is controlled by a barostat algorithm, while the volume of the system is allowed to fluctuate. In this work, $N V T$ ensembles are used to relax the system to an equilibrium state, when one or more observable properties become stable in time. This process is called equilibration phase. During this phase, the temperature is controlled by the Nose-hoover algorithm [54], which acting as a heat bath exchanges the energy with the simulation system. Then, the obtained equilibrium state is used as starting state to perform the simulations in an NPT ensemble during a production phase. This phase is the last step of the simulation, which produce the information of the molecular dynamics.

\subsubsection{Force field}

A force field is constituted of the potential energy functions that describe the different interactions between atoms and the relevant parameters of different atom types, including mass, charge, Lennard-Jones and van der Waals parameters. Since the force field parameters are obtained by QM calculations or experimental measurements, these potential energy functions are also called empirical functions. The combinations of a set of empirical functions can describe the total potential energy of the system as a function of the coordinates of all the atoms present in the system. Until now, a number of force fields have been developed for 
different usage. The choice of force field parameters are crucial for the simulation accuracy.

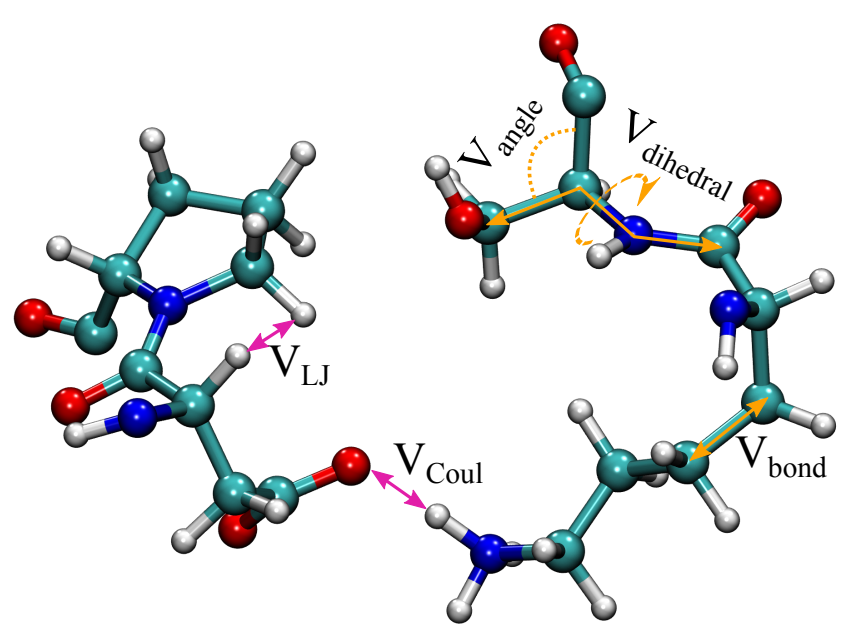

Figure 2.2 A schematic diagram of energy terms in a classical force field. The orange arrows indicate the bonded interactions including bond-stretching potential $\left(V_{\text {bond }}\right)$, angle-bending potential $\left(V_{\text {angle }}\right)$, and dihedral potential $\left(V_{\text {dihedral }}\right)$. The purple arrows point out the non-bonded interactions, namely Lennard-Jones potential $\left(V_{L J}\right)$ and Coulomb potential $\left(V_{C o u l}\right)$.

The general form of a force field is the summation of bonded and non-bonded terms and can be expressed as:

$$
V_{\text {total }}=V_{\text {bonded }}+V_{\text {non-bonded }}
$$

Bonded terms $V_{\text {bonded }}$ represent intra-molecular interactions between atoms that form covalent bonds, comprising bonds $V_{\text {bond }}$, angle $V_{\text {angle }}$, proper and improper dihedral, $V_{\text {dihedral }}$ as illustrated in Fig. 2.2. Non-bonded terms $V_{\text {non-bonded }}$, which are also called non-covalent terms, describe the electrostatic and van der Waals forces (Fig. 2.2). All the energy terms in OPLS force field are defined in the following functional forms:

Covalent bonds are usually modeled as harmonic springs in force fields. Thus, in MD simulations, the bonds cannot be broken but only extended. $V_{b o n d}$ is the interaction energy between atoms $i$ and $j$ forming a covalent bond,

$$
V_{\text {bond }}=\sum_{\text {bonds }} \frac{k_{b}}{2}\left(r-r_{0}\right)^{2}
$$

where $r$ is the distance between the two atoms, $k_{b}$ is the force constant, and $r_{0}$ is equilibrium bond length. 
$V_{\text {angle }}$ is the interaction energy due to the angle $\theta$ formed by three bonded atoms,

$$
V_{\text {angle }}=\sum_{\text {angles }} \frac{k_{\theta}}{2}\left(\theta-\theta_{0}\right)^{2}
$$

where $k_{\theta}$ and $\theta_{0}$ are the angle force constant and equilibrium value of the angle.

$V_{\text {dihedral }}$ represents the energy of a dihedral angel $\varphi$ formed by four bonded atoms,

$$
V_{\text {dihedral }}=\sum_{\text {dihedral }} \frac{V_{n}}{2}[1+\cos (n \varphi-\gamma)]
$$

where $n$ is the multiplicity of the function, $V_{n}$ is the torsional barrier and $\gamma$ is the phase shift.

Out-of-plane bending angle $\omega$ as illustrated in Fig. 2.3 [55] are another class of dihedral angles, also termed improper dihedrals. They can be calculated by Equation 2.6 (in OPLS) or by a harmonic function (in CHARMM) as other bonded terms,

$$
V_{\text {impropers }}=\sum_{\text {impropers }} k_{\omega}\left(\omega-\omega_{0}\right)^{2}
$$

where $k_{\omega}$ is the force constant and $\omega_{0}$ is the equilibrium value of the out-of-plane angle. In addi-

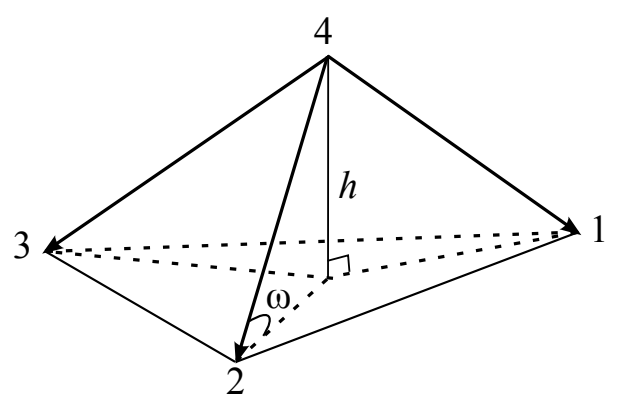

Figure 2.3 A scheme for the out-of-plane bending angle $\omega$. The hight $h$ is the distance between the atom 4 to the plane formed by atoms 1,2 and 3 , where $h \equiv \sin \omega$ tion, a so-called Urey-Bradley angle vibrations is used in the CHARMM force field as a cross-term using 1,3 non-bonded interactions. In this thesis, OPLS-AA force field is used for the simulation of a single protein (Chapter 3) and CHARMM36 is chosen for the simulation in presence of membrane bilayers (Chapter 4).

$V_{L J}$ is the energy function of the Lennard-Jones potential, which is used to describe the van der Waals energy between two atoms $i$ and $j$ found at distance $r_{i j}$,

$$
V_{L J}=\sum_{i} \sum_{j>1} 4 \varepsilon_{i j}\left[\left(\frac{\sigma_{i j}}{r_{i j}}\right)^{12}-\left(\frac{\sigma_{i j}}{r_{i j}}\right)^{6}\right]
$$

where $\varepsilon_{i j}$ and $\sigma_{i j}$ are the depth and the width of the Lennard-Jones potential. 
$V_{\text {coul }}$ describes the electrostatic interaction between two atoms found at distance $r_{i j}$ following Coulomb's Law,

$$
V_{\text {coul }}=\frac{q_{i} q_{j}}{4 \pi \varepsilon_{0} r_{i j}}
$$

where $\varepsilon_{0}$ is the dielectric constant, and $q_{i}$ and $q_{j}$ are the partial charges at atoms $i$ and $j$.

Each atom in the system might be involved in one or more types of interactions. The summation of all the interactions on each atom gives the total potential energy of the system. The pairwise force between atoms $i$ and $j$ at distance $r_{i j}$ can be directly derived by the potential energy $V$ given in Eqs. (2.4) to (2.6), (2.8) and (2.9) with,

$$
F_{i j}=-\frac{\partial V}{\partial r_{i j}}
$$

The total force on an atom, $i$, is thus given as the sum of all forces acting on this atom and expressed as:

$$
F_{i}=\sum_{j} F_{j i}
$$

\subsubsection{Water model}

Water is the solvent of biological systems and provides the natural environment for almost all proteins. Therefore, in order to simulate a system as close as possible to in vivo, the molecular simulations need to include the surrounding water.To illustrate the principles and limitations of classical force fields, this section discusses the structure and force fields of water.

The two hydrogen atoms and the oxygen atom in a water molecule form a 104.48 degree angle instead of the typical tetrahedral angle of 109 degrees. The reason is the presence of two non-binding electron pairs of the oxygen atom, which push the sigma-orbitals of the $\mathrm{O}-\mathrm{H}$ bond closer to each other. The central oxygen atom is partially negatively charged in contrast to the hydrogen atoms, which are partially positively charged. Because of the charge difference, the hydrogen atom is attracted by electronegative atoms, including fluorine, oxygen, or nitrogen, to form a so-called hydrogen bond. Water molecules can form hydrogen bonds with proteins and with themselves. One water molecule can accept and donate two hydrogen atoms by forming maximum of 4 hydrogen bonds. This leads to the formation of a spatial network of hydrogen bonds in the solvent, which is responsible for 
the high melting and boiling temperature of water. Moreover, the effect of solvent on the protein conformation is significant. The forces derived by the solvent, such as the hydrophobic effect, plays a pivotal role in the regulation of protein unfolding. Thus, a proper water model is crucial for MD simulations.

a)

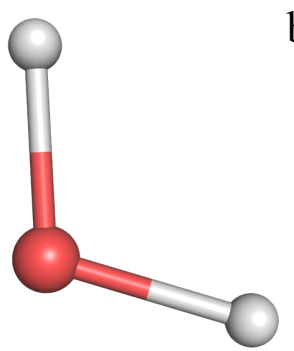

b)

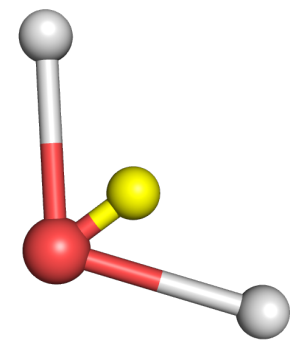

c)

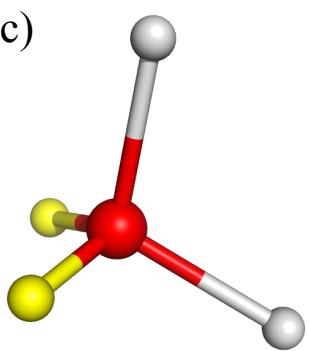

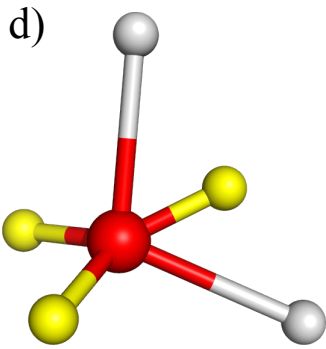

Figure 2.4 This figure shows the general shape of three-site (a), four-site (b), five-site (c) and six-site (d) water models. The oxygen atoms are shown red, the hydrogen are shown in white and the dummy atoms, which only carry a negative charge, are shown in yellow.

Until now, a variety of water models have developed for different kinds of computer simulations of liquid water. For MD simulations of biological systems, a handful of simple water models are usually used (Fig. 2.4). The three atoms in a water molecule are represented as three interaction points in a 3-site model. Each point has a point charge and the same mass as its corresponding atom. Lennard-Jones parameters are assigned to the oxygen atom. Both SPC [56] and TIP3P [57] models belong to this category of water models. The 3-site model is commonly used jointly with protein force fields, such as Charmm [58], Amber [59] and Gromos [60], and provides high computational efficiency.

As described above, the charge difference in a water molecule decides the direction of the dipole moment between oxygen and hydrogen atoms, i.e., intermolecular hydrogen bonding in water requires a correct orientation of water molecules. However, the 3-site model is not able to reproduce the correct orientation because of the inaccurate dipole moment. To improve this, a negatively charged dummy atom is attached the oxygen atom along the bisector of the $\mathrm{H}-\mathrm{O}-\mathrm{H}$ angle resulting in a 4-site model, such as TIP4P [57] (Figure $2.4 b$ ). This dummy atom carries the charge of the oxygen atom, while the oxygen atom only has a mass and Lennard-Jones parameters. The TIP4P model is also implemented in computational chemistry software packages such GROMACS [61]. By combining TIP4P with the OPLS force field [62], it provides accurate reaction environment for biomolecular systems. 
In a 5-site model, such as TIP5P [63], two massless and negative charged dummy atoms are added to represent the two free Ione-pairs of the oxygen atom (Figure $2.4 c$ ). The TIP5P model reproduces the tetrahedral structure of the water molecule, which greatly improves the hydrogen bonding geometry. In the last decade, even a 6-site water model has been developed (Figure 2.4d). However, the more particles are involved in the water model, the higher the computational expense. For a 3-site model, 9 distances between two water molecules $(3 \times 3)$ are required to be calculated to obtain the electrostatic interaction between them. For a 4-site model, one additional distance between the oxygens is required to calculate the Lennard-Jones potential, resulting in 10 distances. Further, for the 5-site model, each water molecule has 4 charge sites and one Lennard-Jones site, thus, $17(4 \times 4+1)$ distances are required, and 26 distances are required for the 6-site model.

It is important to choose a water model with a balance between accuracy and computational cost. In this work, the TIP4P water model with the OPLS-AA force field was used in all simulations in absence of a membrane. CHARMM for protein and membrane together with a modified TIP3P water model was used for the simulations in presence of the membrane. In contrast to the standard normal TIP3P model, this TIP3P water model also places Lennard-Jones parameters on the hydrogen atoms, which makes the calculation two times slower. However, previous studies [64] showed that the CHARMM modified TIP3P water model is crucial for reproducing correct membrane densities. This was also the case in our study (see Chapter 4).

\subsubsection{Applying an external force}

As introduced in Section 1.1, the activation of mechano-sensing proteins is regulated by mechanical force, which induces either protein unfolding or dissociation from an autoinhibitory domain, leading to the exposure of the protein activation site. Single molecule force spectroscopy can be used to investigate such biochemical processes as explained in section 1.2. In this work, MD simulations were carried out to study such force regulated biochemical processes by mimicking the way of force application used in the experiment or thought to be at play in vivo. Such MD simulations are called Force-Probe Molecular Dynamics (FPMD) [65] or Steered Molecular Dynamics [66]. As with AFM, FPMD simulations can be performed with either a constant force of a constant pulling velocity assigned on the desired force application point. 
The limitations of computer resources allow the MD simulation to be performed within a varying length of time from nanoseconds (short simulations) to microseconds (long simulations), or more recently to milliseconds with dedicated hardware [67]. Such time length is usually much shorter than the time length of an unfolding process in vitro. Applying a large mechanical force or a fast pulling velocity on the protein can the unfolding process, however, it can also lead to artifacts. As mentioned in Section 1.2, fast pulling shifts the system far away from equilibrium, i.e. a too high force or a too fast velocity could result in an unrealistic unfolding mechanism. Thus, to reproduce experimental conditions, it is very important to choose a reasonable pulling force or pulling velocity, a tradeoff between computational expense and realistic time scales, which strongly depends on the size and nature of the system under investigation.

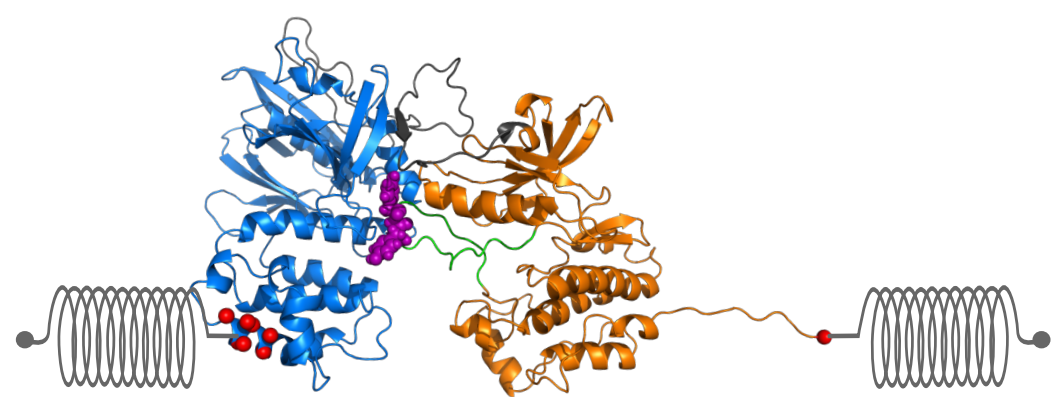

Figure 2.5 FPMD simulation setup of FAK as used in the thesis. The basic patch (red, left) and the $\mathrm{C}$-terminus (red, right) are pulled by virtual springs away from each other inducing unfolding and dissociation.

To reproduce the experimental environment as close as possible, FPMD simulations use the same solvent environment and the same direction and application point of the external force. Fig. 2.5 illustrates how to apply force on a protein in FPMD simulations. A harmonic potential acting as a virtual spring is assigned to each force application point. The force application point can be either a single atom (as the right spring shown in Fig. 2.5) or the center-of-mass (COM) of a set of atoms (as the left spring shown in Fig. 2.5). By moving the virtual spring along the pulling direction with a constant velocity $v$, the mechanical force $F_{i}(t)$, which is eventually induces protein unfolding, can be detected by using the following equation,

$$
F_{i}(t)=k_{0}\left[z_{i}(t)-z_{\text {spring }, i}(t)\right]
$$

where $k_{0}$ is the force constant of the spring, $z_{i}(t)$ is the position of force application point, and $z_{\text {spring, } i}$ is the position of the spring and given as $z_{\text {spring, } i}(t)=z_{i}(0) \pm \Delta z(t)$, where $\Delta z(t)=v t$ 
FPMD simulations were the method of choice for the investigation of force-induced FAK activation (Chapter 4). In the simulations of a single FK-FAK molecule in absence of a membrane bilayer, force was applied to the $\mathrm{C} \alpha$ atom of the kinase $\mathrm{C}$-terminal residue and the COM of the $\mathrm{C} \alpha$ atoms of the basic patch in the FERM domain using constant velocity pulling (Fig. 2.5). In the simulations of the FK-FAK-membrane complex, the force was assigned to the $\mathrm{C} \alpha$ atom of the kinase $\mathrm{C}$-terminal residue and the COM of the membrane bilayer. Due to the harmonic fluctuations of the force application points, the resulting unfolding force varied over a large range with high frequency (black area in Fig. 2.6). Thus, the resulting force signals were smoothed by using Fourier transforms (red line in Fig. 2.6). The highest value in the smoothed force profile was then recorded as rupture force, and is used as a measure for the force required for the dissociation between FAK

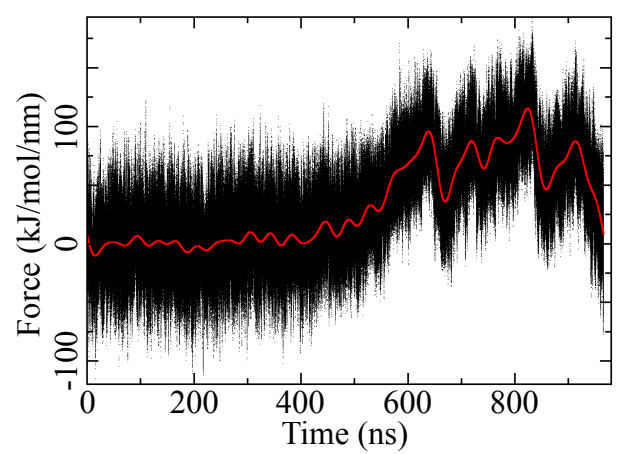

Figure 2.6 An example of a force profile resulting from an FPMD simulation of FKFAK in absence of a membrane. The red line is the smoothed force signal. FERM and kinase domains.

\subsection{Molecular docking}

Molecular docking is an approach to predict a preferred structure of a complex formed by two molecules such as two proteins or a protein and a ligand, which are supposed to bind to each other and form a stable complex (Fig. 2.7). It is widely used in the field of drug design. By calculating the interaction geometry and strength energy between a protein and different ligands, more effective drugs can be developed. The area of a protein, interacting with specific molecules to form complexes, is called a binding site.

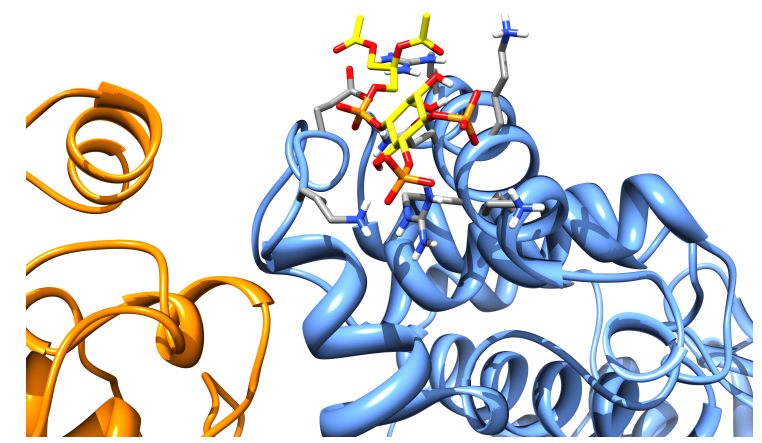

Figure 2.7 The best scored FK-FAK-PIP 2 structure obtained from docking. The highly negatively charged $\mathrm{PIP}_{2}$ binds directly to the highly positively charged basic patch in the FAK FERM F2 lobe

Such binding sites usually have a unique ge- 
ometric shape that can be matched by the complementary shape of another molecule.

A plethora of docking approaches has been developed, that here shall be grouped into two groups, namely rigid docking (lock-and-key [68]) and flexible docking (induced-fit [69]). In the first model, the protein is regarded as a "lock" and the ligand is the "key". Only the key (ligand) with correct size and shape can enter the key hole (binding site) of the lock (receptor protein) in the correct orientation. This model describes the "best fit" orientation of a ligand bound to a particular protein, assuming conformational changes of protein or ligand to be absent. However, since both ligands and molecules are flexible, lock-and-key model cannot explain all experimental findings. Thus, models called "induced-fit" and "conformational selection" [70] were proposed. The "induced-fit" suggests that the binding of a ligand to the target protein is analogous to "hand-in-glove" [71]. During this process, the ligand and the protein adjust their conformation to fit one another. Similarly, protein and ligand in the unbound state straggly fluctuate, and only a subset of conformations is selected for binding.

The evaluation of the predicted structure depends on the strength of the intermolecular interaction and the energy of the complex, which are estimated with the help of a scoring function. Most scoring functions are based on molecular mechanics force fields (see section 2.1.1) to calculate the non-bonded energy between two molecules. A low interaction energy of a complex indicate a stable binding between ligand and protein. The inherent flexibility, leading to induced-fit or conformation selection, needs to be taken into account for accurate docking predictions.

The interactions between two molecules usually belong to four categories, namely, electrostatic forces (Coulombic interaction), electrodynamics forces (van der Waals or Lennard-Jones potential interaction), steric forces (entropy) and solventrelated forces (like hydrophobic effects). According to the nature of the binding be-

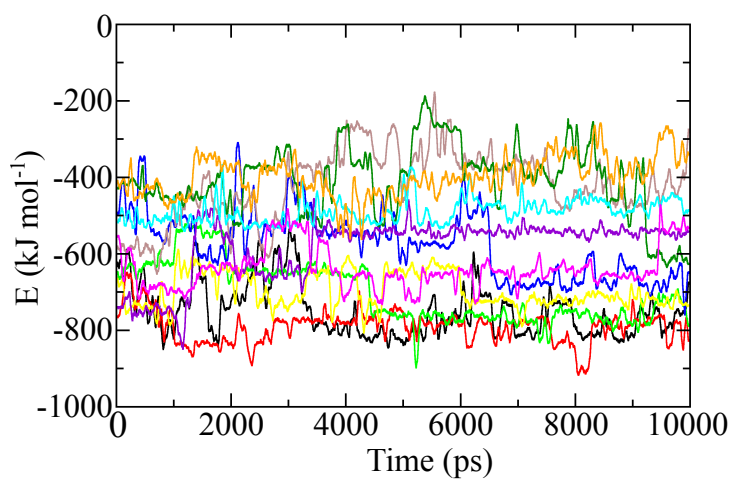

Figure 2.8 The short range coulombic interaction between FAK basic patch and PIP $_{2}$ head group in the ten best complex structures predicted by the UCSF DOCK6.5 suite are calculated by performing MD simulations. tween two molecules, different intermolecular interactions can be selected to score the possible complex poses. In this work, MD 
simulations were used to predict the best geometry of PIP 2 bound to FK-FAK. The basic patch in the FAK F2 lobe is highly positively charged, which binds directly to the highly negatively charged PIP 2 lipid We used the UCSF Dock 6.5 suite (see Section 3.2.1) for flexible docks of $\mathrm{PIP}_{2}$ to the FAK basic patch. The interactions between the two partners were mainly electrostatic nature. To score the obtained docking poses, the short range Coulombic interactions between FK-FAK and the $\mathrm{PIP}_{2}$ head group was calculated during subsequent MD simulations. As shown in Fig. 2.8, Coulombic energies strongly varied. The structure of FK-FAK-PIP 2 complex with the lowest Coulombic interaction energy was chosen as 'best' structure.

\subsection{Force distribution analysis}

The activation and function of mechano-sensing or more generally allosteric proteins are related to the conformational changes induced by an external perturbation such as ligand binding and external forces (Chapter 1). The perturbation gives rise to an internal distribution of this signal in terms of interaction forces, analogously to the stress in a macroscopic structure upon external loading. Thus, understanding the distribution of external mechanical stress within a protein is crucial for revealing the pathway of allosteric signal propagation, which is useful for the prediction of functional mutagenesis and regulation. However, in many cases, the atomic displacements induced by the external perturbation are minor, the site of perturbation and the observed conformational change very distant from each other, which increases the difficulty for most experimental and theoretical methods to observe the complete communication pathway at the coordinate level. Therefore, force distribution analysis (FDA) [72, 73] has been developed in our group for this specific task to detect the changes in forces directly. Analyzing the intramolecular force is a much more sensitive measurement than tracing the atomic displacements [73], because internal forces can be high even when atomic coordinate changes are small, as it is the case in rigid regions of proteins.

FDA provides a variety of output options [73]. The most common one is the scalar pairwise force between atoms or residues. An atomic pair-wise force is defined by the force field according to Equation 2.10. A residue pair-wise force is obtained by vector summation 
of atomic forces as expressed in Equation 2.13,

$$
\vec{F}_{r i, r j}=\sum_{i \in r i, j \in r j} \vec{F}_{i j}
$$

where $i$ is an atom of residue $r i$ and $j$ is an atom of residue $r j$, with $r i$ and $r j$ being different. Angle and dihedral forces are decomposed into pairwise forces between the three or four involved atoms, respectively. Forces from PME are not pairwise and ignored.

In Chapter 3, FDA is used to study the conformational changes of FK-FAK induced by ATP and $\mathrm{PIP}_{2}$ binding. The residue pair-wise forces of different states are collected respectively, and averaged over all simulation steps for each state. A change in pair-wise force reflects the change of internal strain and thus can be considered as a measure for the allosteric effect of ligand binding on the protein structure. The network formed by connecting the residues pairs that have significantly changes in pair-wise forces in different states gives the pathway of allosteric signal propagation upon ligand binding.

The second way is to compute the sum of the time-averaged scalar pairwise forces acting on a residue $r i$ (or an atom):

$$
S_{r i}=\sum_{j}\left|F_{r i, r j}\right|
$$

which measure the total stress acting on the residue ri. This stress is called punctual stress [73] and expressed in units of force. The punctual stress is similar but not equal to other molecular stresses such as the viral stress [74]. Punctual stress can be understood as a very sensitive sensor, which is capable of detecting the "hot-spots" within a complicated network at the atomic level. Mapping the punctual stress as color code onto the corresponding residues provides a direct view of where pair-wise forces accumulate. More details see Chapter 3.

It is often difficult to identify mechanism of the dissociation between two complex molecules such as two protein domains, because there are numerous contacts between them, and the residues at the domain interface swap their partners during the dissociation process. Here, time resolved force distribution analysis (TRFDA) was employed in this work to identify the residues at domain interface, which primarily contribute to the rupture force required for the dissociation between the two domains. Using TRFDA, both pair-wise forces and punctual stresses can be computed at each time step along a trajectory instead of averaged over whole simulation. 
In Chapter 4, TRFDA was carried out to calculate the vector pairwise forces between residues as a function of simulation time. The pairwise force measures the interactions, including short-range Coulomb and Lennard-Jones potentials between residues $r i$ in the FERM F2-lobe and $r j$ in the kinase C-lobe according to Equation 2.13. The punctual stress is calculated by summing up the absolute values of the vector pair-wise forces acting on $r i$ in the $z$,

$$
S_{r i}=\sum_{r j}\left|F_{z, r i, r j}\right|
$$

$S_{r i}$ thus measures the stress acting on the residues located at the domain interface withstanding the external force. The punctual stress here serves as a simple measure for where pair-wise forces accumulate along the pulling direction. TRFDA was carried out on the trajectories obtained from the FPMD simulations, whereas classical FDA is more commonly carried out on the trajectories of equilibrium MD simulations.

\subsection{Principal component analysis}

The structure of a protein is not rigid. In equilibrium, it often deviates from its mead or minimum energy equilibrium structure by showing a variety of dynamic motions in a wide range of time scales, from femtoseconds (bonds vibrations) to microseconds or even longer (protein folding). Large tertiary and quaternary protein conformational changes are often involved in protein function. However, the thermal noise due to the fast harmonic fluctuations make it difficult to identify large scale collective motions of a protein by visual analysis of trajectories. Principal Component Analysis (PCA) has been shown in many studies to be a valuable technique to extract collective motions of biomolecules from MD trajectories.

PCA is a statistical analysis for reducing the dimensions of multivariate datasets with minimal loss of original information. It is based on the covariance matrix, which allows only large-amplitude motions to be detected. The collective motions detected by PCA are called essential dynamics, or principal components (PCs), which argues that only the largest PCs can represent the functional dynamics of a protein such as FAK. Applying PCA on the different protein systems, such as apo and ligand-bound FAK, which we study in Chapter 3 , provides a straightforward insight into the different protein dynamics of different states. Below, the steps of determining the principal components of a trajectory are explained based on the original paper by Amadai at al. [75]. 
For a trajectory of $N$ atoms, the vector $\mathbf{x}(t)=\left(x_{i}(t), y_{i}(t), z_{i}(t)\right)$ describes the coordinates of all the atoms during the simulation, where $i=1,2, \ldots N$ and $t=1,2, \ldots Z$, with $Z$ equal to the length of simulation time. The covariance matrix $\mathrm{C}$, which represents the positional deviations between atomic displacements and the time-averaged structure, $\langle x\rangle$, is expressed as:

$$
\left.C=\langle(x-\langle x\rangle))(x-\langle x\rangle)^{T}\right\rangle
$$

The symmetric matrix $\mathrm{C}$ is then diagonalized by an orthogonal coordinate transformation $\mathrm{T}$ into a diagonal matrix $\Lambda$ with the eigenvalues $\lambda_{i}$ :

$$
C=T \Lambda T^{T} \quad \text { or } \quad \Lambda=T^{T} C T
$$

The $i$ th column of $T$ corresponds to the eigenvectors $u_{i}$ of covariance matrix $\mathrm{C}$, the so called $\mathrm{PC}_{i}$. Each eigenvector $u_{i}$ can be illustrated as $N 3 \mathrm{D}$ vectors, which describe the magnitude and direction of the root mean square (RMS) fluctuation at each atom within the PC $i$. The projection of each frame within the MD trajectories $(\mathrm{P})$ onto each eigenvector $u_{i}$ yields the scaler product of atomic displacements with each eigenvector (Eq. 2.18).

$$
P_{i}(t)=u_{i} \cdot(x(t)-\langle x\rangle)
$$

The variance of the distribution of each resulting projection $\left(P_{i}\right)$ is equal to the eigenvalues $\left(\lambda_{i}\right)$, which describe the total mean square fluctuation of the detected motion (PCi) during the simulation. PCA in this work is carried out with Gromacs tools, g_cover and g_anaeig. Results are given in Section 3.4.

\subsection{Partial least squares functional mode analysis}

Partial least squares functional mode analysis (PLS-FMA) is used to reduce the dimensionality of protein dynamical motions and in this respect is similar to PCA (see above). But in contrast to PCA, PLS-FMA is used to identify not any collective motions but those directly related to a functional property of interest. To this end, PLS-FMA is able to provide a score of the correlation between datasets $\mathrm{Y}$ and $\mathrm{X}$. 
PLS-FMA [76] is a multiple linear least-square regression of the form:

$$
Y(t)=X(t) b+e
$$

It is carried out in this work to identify similar dissociation motions of the FAK FERMkinase fragment in the presence or the absence of the membrane (see Chapter 4). Here, a time-dependent functional variable $Y$ is expressed in terms of the time-dependent 3D atomic coordinates $X(t)$ of the protein or a set of atoms, through a collective (PLS) vector $b$ and residuals $e$. By minimizing the residuals $e$, the correlation between $Y$ and $b$ is maximized. The PLS vector $b$ is established as a linear combination of uncorrelated regressors (PLS components), $k$, which are obtanied via linear combinations of the original coordinates $X$ with maximal covariance with $Y$.

\subsection{Kinetic modeling}

Chemical kinetics concerns the study of rates of chemical reactions. From an aspect of physical chemistry, i.e., the quantitative features of the regulation of a given reaction. From the aspect of biological function, protein kinetics give insight into biological processes such as cell signaling. Biological processes often consist of an extensive chain of chemical reactions, which often are kinetically regulated by one or few certain steps, such as the signaling cascade triggered by FAK activation. In our case, a kinetics model can provide information about the influence of mechanical force on the system at different levels of organization, from the single step of FAK catalytic turnover to the formation of multiple protein complexes of FAK with downstream proteins, all the way down to the phosphorylation of Ras GTPase.

To characterize a chemical reaction or biological process, three aspects of this reaction need to be considered. The first is the influence of different experimental conditions, including temperature, pressure, concentrations, reaction medium and catalysts, on the rates of a chemical reaction. The second is the reaction mechanism, which defines how many steps and transition states exist in the reaction process. The third is to choose proper reaction laws, which describe the change of the reaction rate upon the concentration of reactants. The systematic collection of these information is the prerequisite for the construction of a mathematical model to describe the reactions. The kinetic model of a simple mechanism of 
an enzymatic process is presented as an example in the text below.

In biochemistry, a simple mechanism of enzymatic process is written as:

$$
\mathrm{E}+\mathrm{S} \underset{k_{-1}}{\stackrel{k_{1}}{\rightleftharpoons}} \mathrm{ES} \underset{k_{-2}}{\stackrel{k_{2}}{\rightleftharpoons}} \mathrm{EP} \underset{k_{-p}}{\stackrel{k_{p}}{\rightleftharpoons}} \mathrm{E}+\mathrm{P}
$$

where enzyme (E) binds to a substrate (S) to form a transition complex (ES), which can rapidly be transformed to another transition complex (EP). Then, EP can be very fast converted into a product $(\mathrm{P})$ and the enzyme. The forward (and backward) rates of the three steps, $k_{1}, k_{2}$ and $k_{p}$ (as well as $k_{-1}, k_{-2}$, and $k_{-p}$, respectively describe the kinetics of the overall reactions. In this catalytic process, the enzyme acting as a catalyst just accelerate the reaction by lowering the activation energy without affecting the reaction equilibrium.

To describe a reaction in dynamic equilibrium such as $\mathrm{S} \rightleftharpoons \mathrm{P}$, Peter Waage and Cato Guldberg formulated the law of mass action in 1864, which initiated the development of chemical kinetics. According to this law, the equilibrium can be described by an equilibrium constant, $K_{e q}$, which is expressed as: $K_{e q}=[P] /[S]=k_{1} / k_{-1}$. The free energy difference between $\mathrm{S}$ and $\mathrm{P}$ is expressed as: $\Delta \mathrm{G}^{0}=-\mathrm{RT} \ln K_{e q}$.

The rate of a reaction or process is defined as appearance of products $[\mathrm{P}]$ per unit time and is determined by the multiplication between the rate constant, $k$, and the concentration of the reactant (or reactants). For a reaction as:

$$
n_{A} \mathrm{~S}_{1}+n_{B} \mathrm{~S}_{2} \stackrel{\mathrm{k}}{\longrightarrow} \mathrm{P}
$$

the reaction rate is given as $v=\mathrm{d}[\mathrm{P}] / \mathrm{dt}=\mathrm{k}\left[\mathrm{S}_{1}\right]^{a}\left[\mathrm{~S}_{2}\right]^{b}$. The sum of a and $\mathrm{b}$ is called the order of reaction, which can be measured by experiments. For $a+b=1$, the reaction is called the first-order reaction, the reaction rate $\mathrm{k}$ has units of $\mathrm{s}^{-1}$. For $\mathrm{a}+\mathrm{b}=1$, it is called the second-order reaction, the reaction rate $\mathrm{k}$ has units of $\mathrm{M}^{-1} \mathrm{~s}^{-1}$.

The Mechaelis-Menten mechanism is one of the best-known models to describe an enzymatic process. It is based on two assumptions. The first assumption is that the reverse reaction $(\mathrm{P} \longrightarrow \mathrm{S})$ is negligible, because the product is assumed to immediately interact with downstream proteins (or other species). Based on this assumption, the enzymatic mechanism can be simplified to:

$$
\mathrm{E}+\mathrm{S} \underset{k_{-1}}{\stackrel{k_{1}}{\rightleftharpoons}} \mathrm{ES} \stackrel{k_{c a t}}{\longrightarrow} \mathrm{E}+\mathrm{P}
$$


Applying the reaction law gives a set of non-linear ordinary differential equations (ODEs) that describe the changes in concentrations of reactants over time.

$$
\begin{aligned}
\frac{d[E]}{d t} & =-k_{1}[E][S]+k_{-1}[E S]+k_{c a t}[E S] \\
\frac{d[S]}{d t} & =-k_{1}[E][S]+k_{-1}[E S] \\
\frac{d[E S]}{d t} & =k_{1}[E][S]-k_{-1}[E S]-k_{c} a t[E S] \\
\frac{d[P]}{d t} & =k_{\text {cat }}[E S]
\end{aligned}
$$

To solve these equations, another very important assumption is required, the quasi-steadystate assumption. It assumes that the concentration of any intermediates such as [ES] remains constant over time, i.e., $d[E S] / d t=0$. Since $[\mathrm{ES}]+[\mathrm{E}]=[\mathrm{E}]_{0}$ according to the enzyme conservation law, the concentration of the complex [ES] becomes:

$$
[E S]=\frac{[E]_{0}[S]}{K_{m}+[S]}
$$

where,

$$
K_{m}=\frac{k_{-1}+k_{c a t}}{k_{1}}
$$

is known as the Michaelis constant. Hence, the rate $v$ of the reaction is solved and expressed as:

$$
v=\frac{d[E S]}{d t}=\frac{v_{\max }[S]}{K_{m}+[S]}
$$

where $v_{\max }=k_{\text {cat }}[\mathrm{E}]_{0}$.

Similar to the construction of the mathematical model of an enzymatic process, a kinetic model of FAK signaling has been formulated in this thesis. Kinetic modeling is based on an extensive collection of experimental data. However, the force-dependent rate of FAK mechanical activation has not yet been identified experimentally. We here obtain the kinetic parameters from MD simulations (see Chapter 4). By performing MD simulations, we investigated the mechanism of FAK mechanical activation, which switch on the activation of downstream proteins. Applying the DHS model (see Section 1.3) gives a force-dependent reaction rate (Equation 1.3) of FAK catalytic turnover. Combining this reaction step with the downstream reactions kinetics of which has been quantified in previous studies, a mechanobiochemical network of FAK signaling was constructed. Each chemical species in the net- 
work is represented by an ODE, which is used to trace the dynamics of the concentrations of chemical species over time. The ODEs are solved by using COPASI. COPASI is free software package for modeling and simulations of biochemical networks [77]. Analysis of the results, in particular the force dependency of downstream Ras signaling, is presented in Section 4.8. 


\section{CHAPTER 3}

\section{ALLOSTERIC REGULATION OF FAK BY LIG- ANDS BINDING}

This chapter focuses on the dynamics and structural changes of the FAK FERM-kinase fragment induced by ATP and additional PIP $_{2}$ binding. As showed by recent fluorescence resonance energy transfer (FRET) experiments, ATP binding results in a closure of the FERM-kinase interface, which is reversed by additional $\mathrm{PIP}_{2}$ binding [41]. To reveal the atomistic details of this open-to closed motion of FAK, a series of all-atom MD simulations was perfomed in the absence or presence of ATP and $\mathrm{PIP}_{2}$ followed by principal component analysis (PCA) and force distribution analysis (FDA).

\subsection{Introduction}

Chapter 1 presents an important role of FAK as a cellular mechano-sensor at the crossroads of multiple signaling pathways. In short, FAK is activated at focal adhesion sites through protein-protein interactions of its C-terminal FAT domain with intergrin and actinassociated proteins, such as paxilin and talin $[14,78,79]$. The activation of FAK regulates the formation and function of FAs, which enables the integration of mechanical and biochemical signals between the outside and inside of cell and establish a mechanical link of the cellular cytoskeleton with the ECM [14]. Thus, FAK is crucial for the regulation of multiple cellular processes, including cell signaling, proliferation, migration and development. 
Understanding its mechanism of activation and function are of pivotal importance for the rational design of new treatments of severe human diseases, in particular treatment-resistant cancers [80]. To reveal the mechanism of FAK activation, many stimuli have been investigated. Recent study showed that the direct binding of $\mathrm{PIP}_{2}$ to the basic patch in the FERM domain can induce partial activation of FAK $[42,44]$. Therefore, the study of $\mathrm{PIP}_{2}$ induced FAK allostery might be helpful for revealing the mode of FAK activation.

In order to achieve this goal, biochemical, structural, and FRET experimental studies have recently been carried out [41]. The experimental results shows an increased FRET efficiency measured between the N-termini of the kinase N-lobe and the FERM F1-lobe induced by the binding of ATP to the activation loop of the kinase domain, which suggests a closure of FERM-kinase interface. In contrast, the conformational rearrangement induced by additional $\mathrm{PIP}_{2}$ binding to a basic patch of FERM-F2 results in an even lower FRET efficiency than FK-FAK in absence of ligand binding, indicating a partially opened

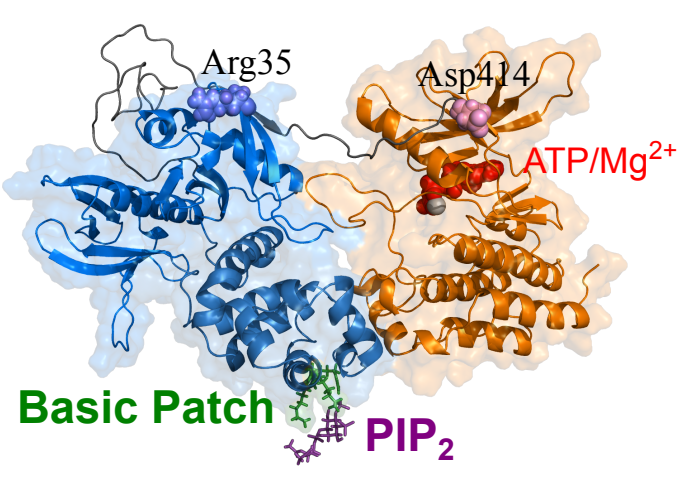

Figure 3.1 Molecular structure of FAK FERMkinase fragment. The positions of ATP (red and $\mathrm{PIP}_{2}$ (purple) are shown at representative positions, as observed during the simulations. Sites of FRET labels (Arg35 and Asp414, shown as sphere) and the basic patch dark green are highlighted.

$\mathrm{PIP}_{2}$-bound FERM-kinase fragment (FK-

FAK) structure. These conformational transitions upon ATP and $\mathrm{PIP}_{2}$ binding enhanced the efficiency of auto-phosphorylation of Tyr397 at FAK linker. However, binding was still not able to induce sufficient conformational changes required for the phosphorylation of the key tyrosine residues (Tyr576/577) resulting in maximum activation of the kinase domain.

Molecular simulations can provide direct insight into the underlying molecular mechanism of such allosteric effects at high spatial and temporal resolution, which cannot be achieved by biological experiments. In this study, only the FERM and kinase domains of FAK were considered. The structure and dynamics of FK-FAK in three systems have been studied. These were: (1) FK-FAK in absence of ligands (apo-FK); (2) FK-FAK bound to ATP (FK-ATP); (3) FK-FAK bound to ATP and PIP 2 (FK-ATP-PIP 2 ). By performing a series of MD simulations, a model of ATP and PIP 2 -bound FAK complexes at atomistic scale have been proposed. These models have been based on previous structural and muta- 
tional data (Figure 3.1), which detected distinct long-range conformational changes in the kinase and FERM domains. Then, global motions of FAK fragments were analyzed using PCA (see Section 2.4). We have found that ATP binding leads to closure of the two kinase lobes, which is reversed by subsequent $\mathrm{PIP}_{2}$ binding with structural consequences at the FERM-kinase interface. Next, FDA (see Section 2.3) has been carried out in order to characterize the stress distribution in the FAK protein upon ligand binding, allowing to identify the allosteric network of primarily electrostatic interactions. This network spans from the $\mathrm{PIP}_{2}$ binding site to the distant interface between the kinase N-lobe and the FERM F1-lobe and comprised candidate residues for site-directed mutagensis experiments to validate the mechanism proposed herein.

\subsection{Materials and Methods}

\subsubsection{Molecular Docking}

For all simulations, the crystal structure (PDB code: 2J0J) of the FAK fragment comprising the FERM and kinase domain (residues 35-686) [36] was used as starting structure. Coordinates of flexible loops, including those of residues 362-394, 402, and 574-583, were not resolved and were completed by MODBASE [81]. The structure of FK-ATP was obtained by inserting $\mathrm{ATP} / \mathrm{Mg}^{2+}$ in the same binding pose resolved in the crystal structure of FK-FAK in complex with ANP (PDB: 2J0L) [36]. The initial geometry of the FK-ATP-PIP 2 complex was obtained using a molecular dock-

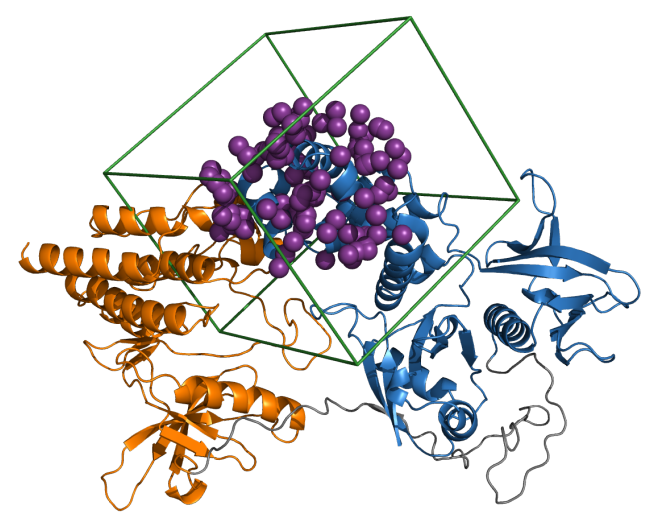

Figure 3.2 The system of grid-based molecular docking. The FAK fragment is shown as cartoon colored by corresponding colors, and the force field grid is outlined by the white box. The violet Balls indicate sphere of the Binding pocket. ing procedure, with the putative $\mathrm{PIP}_{2}$ binding site assigned to the FERM basic patch (residues 216-222). Partial atomic charges on the $\mathrm{PIP}_{2}$ molecule were assigned using the RESP methodology [82-84] and obtained by REDS [82, 83], using HF/6-31G* as basis set. The total charge on the $\mathrm{PIP}_{2}$ head group 
was set to -5 , which corresponds to the fully deprotonated state, since potential protons of $\mathrm{PIP}_{2}$ in its most common state with -4 as a total charge [85] are likely to be displaced by Lys/Arg interactions upon binding to FAK. Molecular docking was performed with the UCSF DOCK6.5 suite [86], using the semi-rigid docking procedure and energy grid scoring in an implicit solvent. The grid spacing was $0.025 \mathrm{~nm}$, and the grid included $1.2 \mathrm{~nm}$ beyond the FAK basic patch (Fig. 3.2). The energy score was the sum of electrostatic and van der Waals contributions. In the course of the docking procedure, the $\mathrm{PIP}_{2}$ molecule was subjected to 2500 cycles of molecular-mechanical energy minimization. The number of maximum ligand orientations was 5000. The best-scoring 25 FK-ATP-PIP 2 complexes were further analyzed by means of MD simulations. The best structure was chosen according to the most favorable Coulombic interaction between the $\mathrm{PIP}_{2}$ head group and the basic patch in the FERM domain.

\subsubsection{Molecular Dynamics simulations}

All MD simulations were carried out using GROMACS 4.0.5 [61]. The OPLS allatom force field [62] for the protein and the TIP4P water model [57] were used. The temperature was kept constant at $\mathrm{T}=300 \mathrm{~K}$ by using velocity rescaling with a coupling time of $0.1 \mathrm{ps}$. The pressure was kept constant at 1 bar using an isotropic coupling to Parrinello-Rahman barostat with a coupling time of $0.1 \mathrm{ps}$ [87]. A cut-off of $1 \mathrm{~nm}$ was used for all non-bonded interactions. Long-range electrostatic interactions were treated with the PME [88] method using a

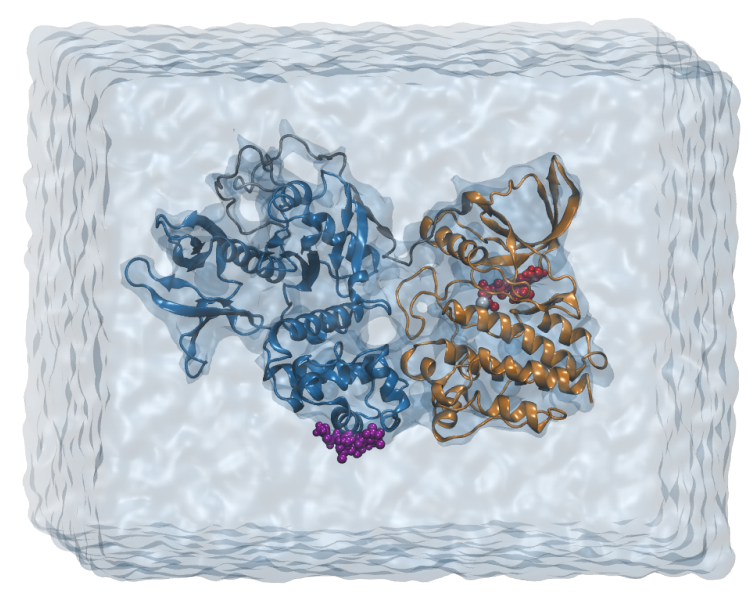

Figure 3.3 The cubic simuation box contains protein with ligands solved TIP4P water and neutralized by sodim and chloride ions. grid spacing of $0.12 \mathrm{~nm}$ with cubic interpolation. All bonds between hydrogens and heavy atoms were constrained using the LINCS algorithm [53]. An integration time step of 2 fs was used. During all simulations, the neighbor list was updated every 10 steps.

Each of the three structures, apo-FK, FK-ATP and FK-ATP-PIP 2 , were immersed in a cubic TIP4P water box containing about 200,000 atoms (Figure 3.3). Sodium and chlo- 


\subsection{ATP and PIP2-induced conformational changes of FAK}

ride counter-ions ( $0.1 \mathrm{M}$ concentration) were added to neutralize the system. Prior to the MD simulations, all investigated systems were energy-minimized using the steepest descent method for 10000 steps. This was followed by 2 ns MD simulations, during which position restraints were used on all protein atoms. Finally, each system was equilibrated during $200 \mathrm{~ns}$ MD simulations. The last $120 \mathrm{~ns}$ trajectories of each simulation were used for analysis.

\subsection{ATP and PIP2-induced conformational changes of FAK}

In order to characterize the interactions between ATP, PIP 2 and FAK at atomistic detail, MD simulations of following systems have been performed: (1) the FAK fragment containing the FERM and kinase domain (apo-FK), (2) the same fragment with ATP bound (FK-ATP) and (3) the same fragment bound to ATP and PIP $_{2}$ (FK-ATP-PIP 2$)$ (Figure 3.1). All simulations were carried out under the same conditions. It should be noted that that the FK-ATP-PIP 2 complex contained only the $\mathrm{PIP}_{2}$ head group (C2-PIP $)$, not the whole lipid. This is justified, since the hydrophobic tail of $\mathrm{PIP}_{2}$ is supposed to be embedded in the membrane and does not contribute to the proteinlipid interaction. The interaction of the FKFAK fragment with the PIP $_{2}$ enriched membrane is described in Chapter 4. In a solution with $\mathrm{pH} 7, \mathrm{PIP}_{2}$ carries most likely a net-charge of -4 , which corresponds to the

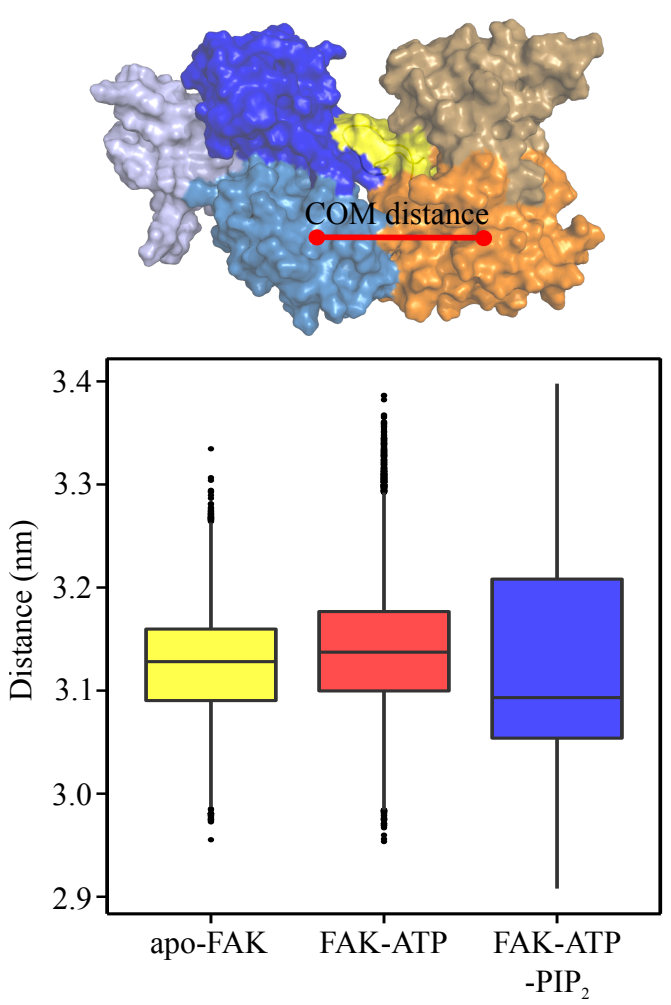

Figure 3.4 Average and deviations of the distance between the centers of mass of the FERM F2-lobe and the kinase C-lobe are shown. ATP and $\mathrm{PIP}_{2}$ binding do not effect the F2-C interface singly-protonated state. [85]. However, in our simulations the net charge of -5 , corresponding to the fully-protonated state, has been assigned to the $\mathrm{PIP}_{2}$ head group. The reason for this was that the proton bound to $\mathrm{PIP}_{2}$ is likely displaced upon binding the FAK [85]. 
In all simulations, comprising $3 \times 0.2 \mu$ s for each state, there was no sign of a full dissociation of the inhibitory FERM domain from the catalytic kinase domain. Instead, the distance between the FERM F2-lobe and the kinase C-lobe remained unchanged during the whole simulation (Figure 3.4). However, some allosteric conformational changes at the interface of FERM and kinase domans induced by ATP and PIP $_{2}$ binding were observed. To validate these conformational changes and to compare them with FRET data [41], the distances between Arg35 (the N-terminal residue of the FERM domain) and Asp414 (the $\mathrm{N}$-terminal residue of the kinase domain), which were the labeling sites for FRET sensors, were computed for the MD simulation trajectories (Figure 3.5a).

a)
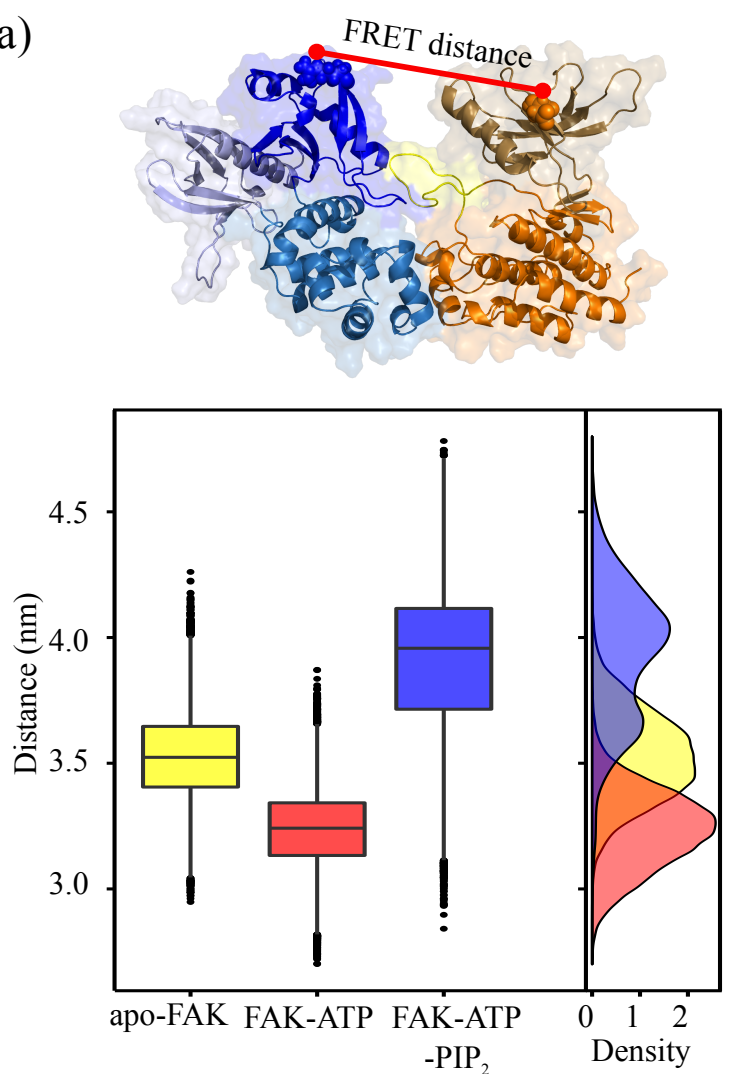

b)
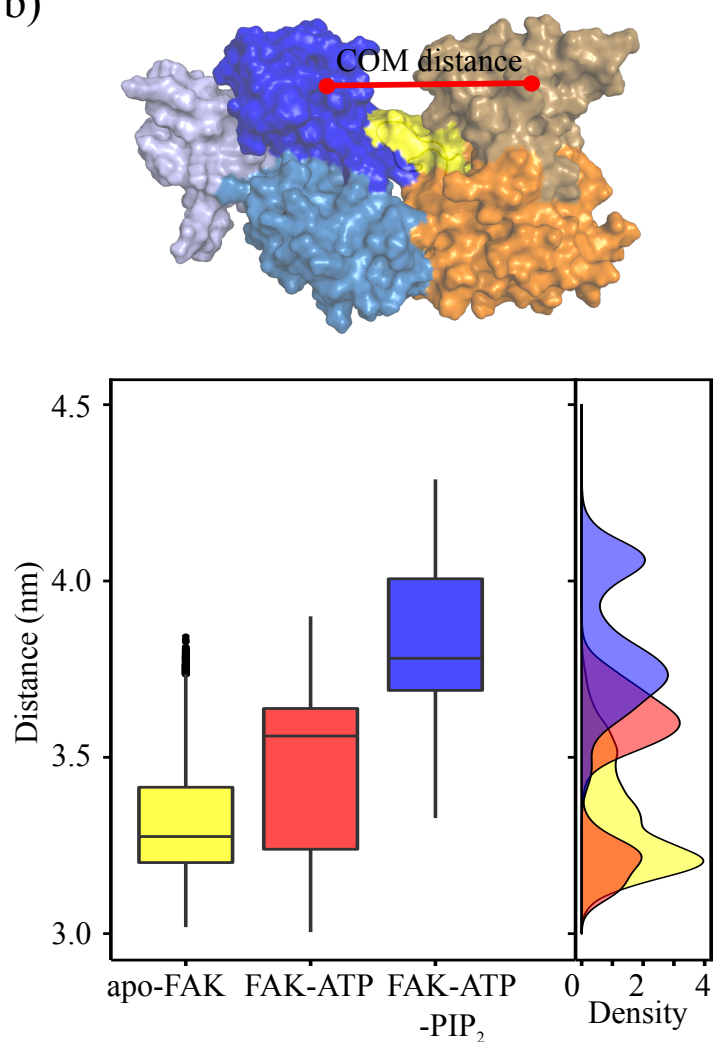

Figure 3.5 Conformational changes upon ATP/PIP 2 binding measured by inter-domain distances as observed in MD simulations of the three states of FAK, namely apo-FAK (yellow), FK-ATP (red) and FK-ATP-PIP 2 (blue). (a) Distribution (right) and deviations (left) of distance between the residues where FRET labels were inserted in the FERM and kinase domain. b) Distribution (right) and deviations (left) of distance between the centers of mass of the FERM F1-lobe and the kinase Nlobe.

As shown in Fig $3.5(a)$, the shortest median FRET distance for FK-ATP was about $3.2 \mathrm{~nm}$, whereas the FRET distances of apo-FK and FK-ATP-PIP 2 were equal and above 


\subsection{ATP and PIP2-induced conformational changes of FAK}

$3.5 \mathrm{~nm}$, respectively. This trend is in very good agreement with the experimental data, which gave a larger FRET efficiency for ATP-bound FAK and a lower FRET efficiency for ATP$\mathrm{PIP}_{2}$-bound complex as for apo-FK [41]. However, the distances between the FRET labels cannot reflect the inter-domain distance. As can be seen in Fig 3.5 b, more than $50 \%$ of apoFAK center-of-mass (COM) distances between the N-lobe and F1-lobe were in the range of 3.1 of $3.3 \mathrm{~nm}$, which is shorter than the median COM distance of FK-ATP (3.5 nm) and FKATP-PIP $_{2}(3.8 \mathrm{~nm})$. Thus, the binding of ATP to apo-FAK induces an apparent closure in the simulations, similar to what was measured by the FRET labels in experiments. However, the experimental results did not show an opening of the respective sub-domains, the N-lobe and F1-lobe, relative to each other. In contrast, PIP $_{2}$ binding to FK-ATP resulted in a partial opening at the interface between N-lobe and F1-lobe leading to increased distances of both the COM of the two domains and the respective FRET labels. Thus, the addition of $\mathrm{PIP}_{2}$ to FAK could induce the switch from an inactive to an active kinase domain.

To understand the effect of ATP and PIP 2 on the FK-FAK, the backbone fluctuations of FERM and kinase domain in all simulations were calculated and averaged over the trajectories for each state. Figure 3.6 shows the changes of residue fluctuations within the FERM F2-lobe upon on ATP and $\mathrm{PIP}_{2}$ binding. The highlighted areas indicate the basic patch (light green) and a loop (residue: 180-190, light violet) at the F2/C interface. This loop seems to be disordered in the crystal structure (PDB ID: 3ZDT) [41] of the FERM domain in absence of the kinase domain (F-FAK) containing charge-neutralized mutations of the basic patch. In the experiment, the basic patch mutation was meant to mimic the effect of $\mathrm{PIP}_{2}$. However, in MD simulations, the fluctuation of this lobe is just slightly increased in FK-ATP-PIP 2 . Instead, the fluctuations of residues between these two highlighted areas at the domain interface are overall increased in FK-ATP.

A plausible explanation is that ATP binding can induce long-range rearrangements of the F2/C-lobe interface, which reduces the interaction between the FERM

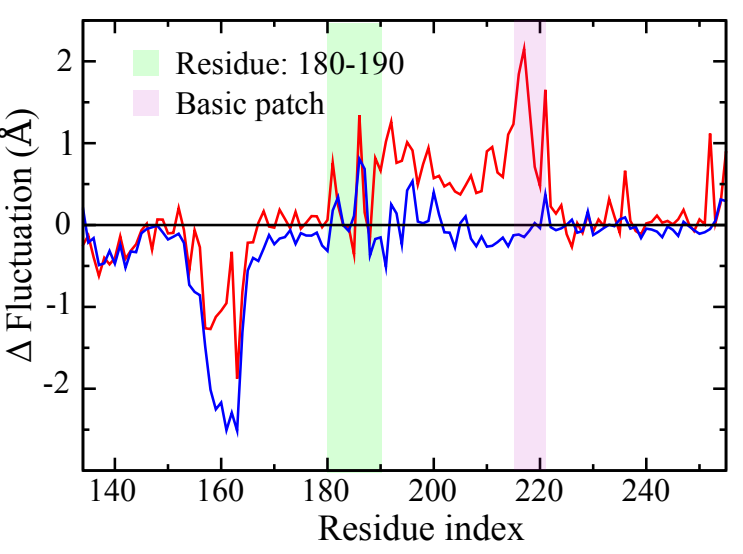

Figure 3.6 The changes of Root mean square fluctuations (RMSFs) of FERM F2-lobe upon ATP and $\mathrm{PIP}_{2}$ binding. Differences in RMSFs to the apo-state as reference are shown in red for FKATP and in blue for FK-ATP-PIP 2 . F2- and kinase C-lobe and consequently 
leads to a high flexibility of the residues within the F2-lobe in FK-ATP as compared to F-FAK. The additional basic patch mutant in F-FAK attenuates the interactions of the basic patch with its adjacent helices and induces further destabilization (or disorder) of the loop 180-190. Similar to the basic patch mutant, additional $\mathrm{PIP}_{2}$ binding neutralizes the charges on the basic patch and reduces the electrostatic interactions of the basic patch with its adjacent helices. At the same time, it also enhances the rigidity of F2-lobe by forming salt-bridges with the basic patch and its adjacent helices, inducing a rearrangement of the F2/C-lobe interface.

a)

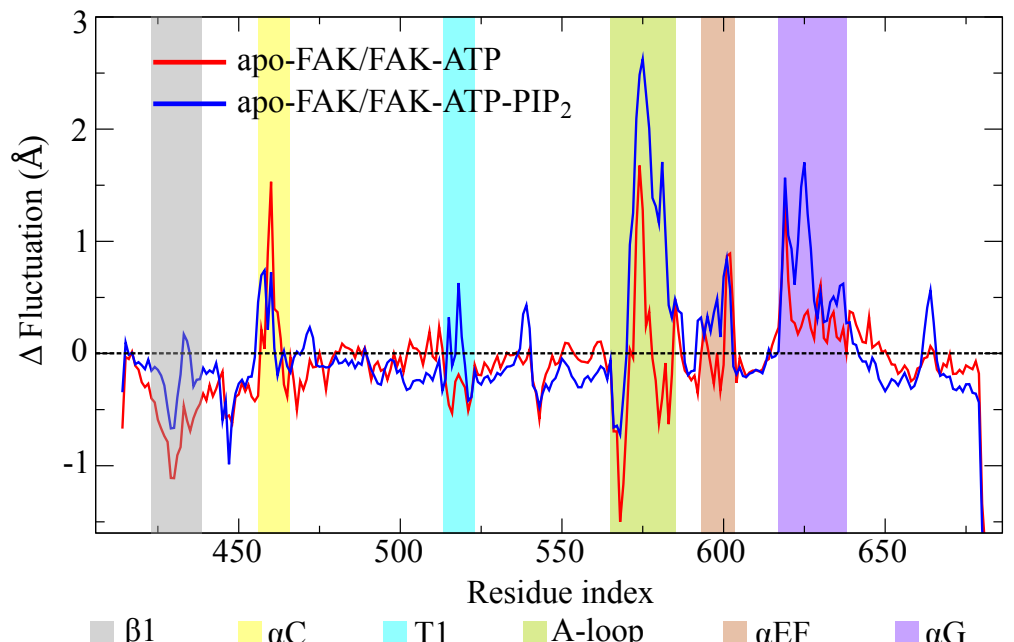

b)

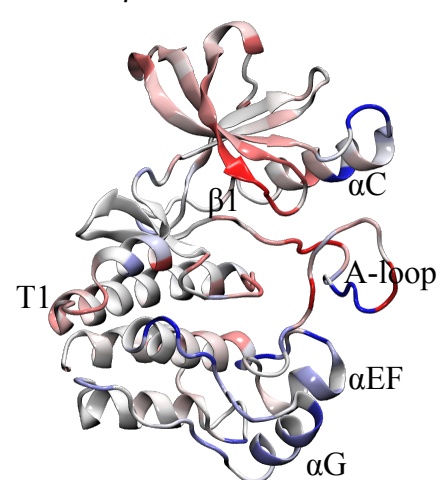

apo-FAK/FAK-ATP

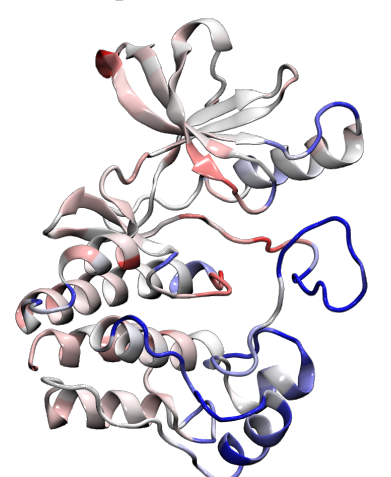

apo-FAK/FAK-ATP-PIP 2

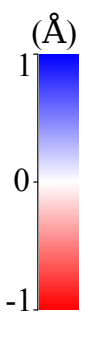

Figure 3.7 RMSFs of the kinase domain upon ATP and PIP 2 binding. (a) Differences in RMSFs to the apo-state as reference are shown in red for FK-ATP and in blue for FK-ATP-PIP 2 . Regions with large significant changes of residue fluctuations have been labeled with different colors. (b-c) Kinase domains are colored according to the differences in RMSF shown in (a).

Next, changes of residue fluctuations within the kinase domain (Figure 3.7a) were analyzed. The kinase domains in FK-ATP (Figure 3.7b left) and in FK-ATP-PIP 2 (Figure $3.7 b$ right) were colored according to the differences in RMSFs to the apo-state as a reference. 


\subsection{Detection of functional modes in FAK dynamics}

The presence of ATP in the active site leads to a maximal stabilization of the $\beta \mathrm{C}$-sheet and the activation loop as shown in Figure 3.7b left. It also increased the flexibility of $\alpha \mathrm{C}-, \alpha \mathrm{EF}$ and $\alpha \mathrm{G}$-helices. The binding of $\mathrm{PIP}_{2}$ reduced the stabilization of the $\beta \mathrm{C}$-sheet and the activation loop induced by ATP binding and further enhanced the fluctuations of the activation loop, $\alpha \mathrm{C}$-, $\alpha \mathrm{EF}$ and $\alpha \mathrm{G}$-helices. All these sites are located at the domain interface and are part of the auto-inhibitory interaction sites of the kinase domain with the FERM domain. Overall, the allosteric effects of ATP and $\mathrm{PIP}_{2}$ on the active site of the kinase domain appear to be of opposite nature, not only in terms of inter-lobe distances (Figure 3.5), but also in terms of fluctuations (Figure 3.7).

To summarize, we propose that the binding of ATP and $\mathrm{PIP}_{2}$ results in changes at the FERM F2/kinase C-lobe interface. These affect the rigidity at the FERM-kinase interface, as suggested by the RMSF, and allows for the allosteric open-to-closed motion between the $\mathrm{N}$-lobe and F1-lobe, as suggested by the distance measurements. In none of the three states examined, the phosphorylation site Tyr576/577 was released from the FERM domain. These observations strongly suggest that the FRET-sensors only measured partial FAK activation. The validation of the observed ATP and $\mathrm{PIP}_{2}$ dependent dynamics in FAK by comparison to FRET experiments allows for functional analysis of the ligand-dependent dynamics.

\subsection{Detection of functional modes in FAK dynamics}

To analyze the functional motions underlying the observed distance changes and the opposite allosteric effects of ATP and $\mathrm{PIP}_{2}$ at the active site in the kinase domain, a principal component analysis (PCA) [89] of the kinase domain in the apo state was carried out. Two major motions were observed, as reflected by the projection of the MD trajectory on the first two eigenvactors, which accounts for $\sim 45 \%$ of the overall protein motion (Figure 3.8a). As shown in Figure 3.8b, eigenvalue 1 (EV1) represents an open-to-closed motion between the $\mathrm{C}$ - and N-lobe, and EV2 corresponds to a twisting motion of the FAK kinase domain. To study the differences between the protein's conformational motions in its three states, the trajectories of the kinase domain in the FK-ATP and FK-ATP-PIP 2 states were projected onto the eigenvectors calculated for the apo state.

As can be seen in Figure 3.9, apo-FK samples a broad range of conformations, including both the open-to-closed inter-domain motion and the intra-domain twisting motion (yellow). 


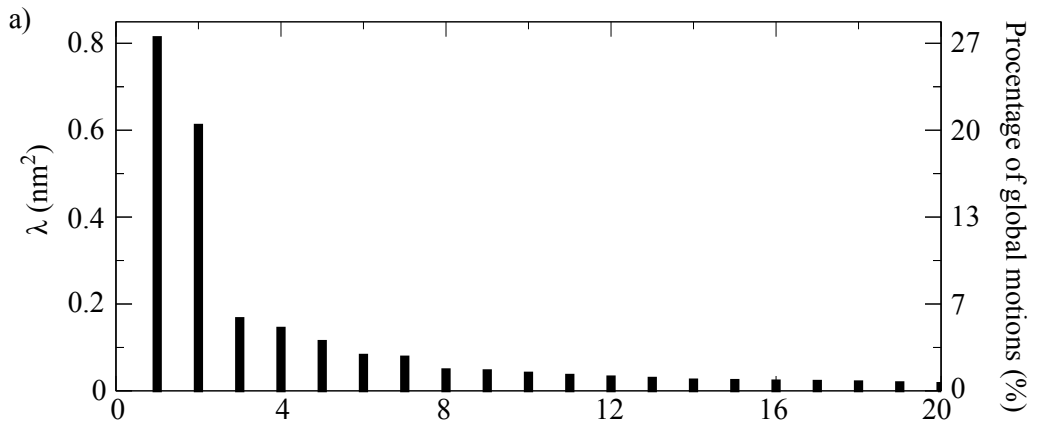

b)

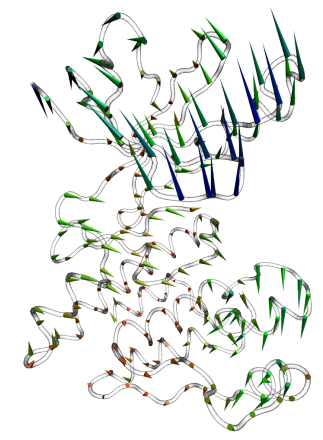

Eigenvector index

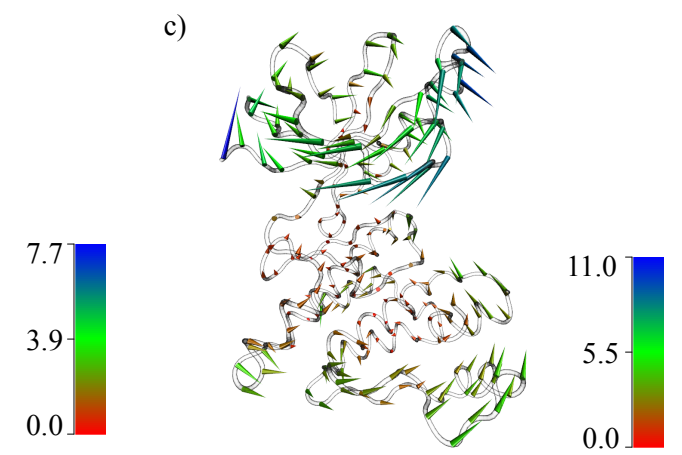

Figure 3.8 Collective motions of the kinase (a) 20 largest eigenvalues from PCA of kinase domain $\mathrm{C}_{\alpha}$ atoms as observed for apo-FAK. The first two modes describe $45 \%$ of the total motion of this domain (b-c) Porcupine plot of the FAK kinase domain, illustrating the motion along the first (b) and second (c) eigenvectors (EV) obtained from PCA. Coloring according to the deviation in eigenvalue between the two extreme structures along the respective EV. EV1 (b) describes an opening, EV2 (c) a twisting motion.

The binding of ATP between the kinase $\mathrm{N}$ and C-lobe restricted the open-close transition and constrained the residues in the active sites. PIP $_{2}$ binding to the basic patch in the FERM domain, in turn, released some of these constraints, and the kinase domain was found in a more open conformation (negative eigenvalues of EV1). These results are in agreement with the RMSF measurements (Figure 3.7) and can explain the increased distances observed experimentally (Figure 3.5).

In addition, the available X-ray structures were also projected onto the collective coordinates (Figure 3.9). The experimentally resolved crystal structures, including the FERMkinase domain in the presence of staurosporine (PDB:2J0J, violet) and the ANP bound kinase domain (2J0L, green) [36] lie well within the dynamics observed in our MD simulations for three states. For comparison, a number of structures of the true apo-state of the kinase in absence of the auto-inhibitory domain or any ligand bound to the active site (black), reproduced the large variability of the apo state along EV1. Along EV2, they dis- 
tributed in the conformational space observed in the MD simulations for FK-ATP and FK$\mathrm{ATP}_{\mathrm{PIP}}$. A possible interpretation is that the interaction of kinase domain with FERM domain leads to an apparent shift of the twist in the kinase domain, which can be reversed by dissociation of the kinase from the FERM domain or by weakening of the FERM-kinase interface upon ATP and $\mathrm{PIP}_{2}$ binding. It should be emphasized that until now there is no experimental structure of FK-FAK in the apo state available. All crystal structures were obtained either with a bound ligand or in absence of the FERM domain. Therefore, a direct comparison between our simulated FK-FAK fragment and the experimental structures is not straightforward.
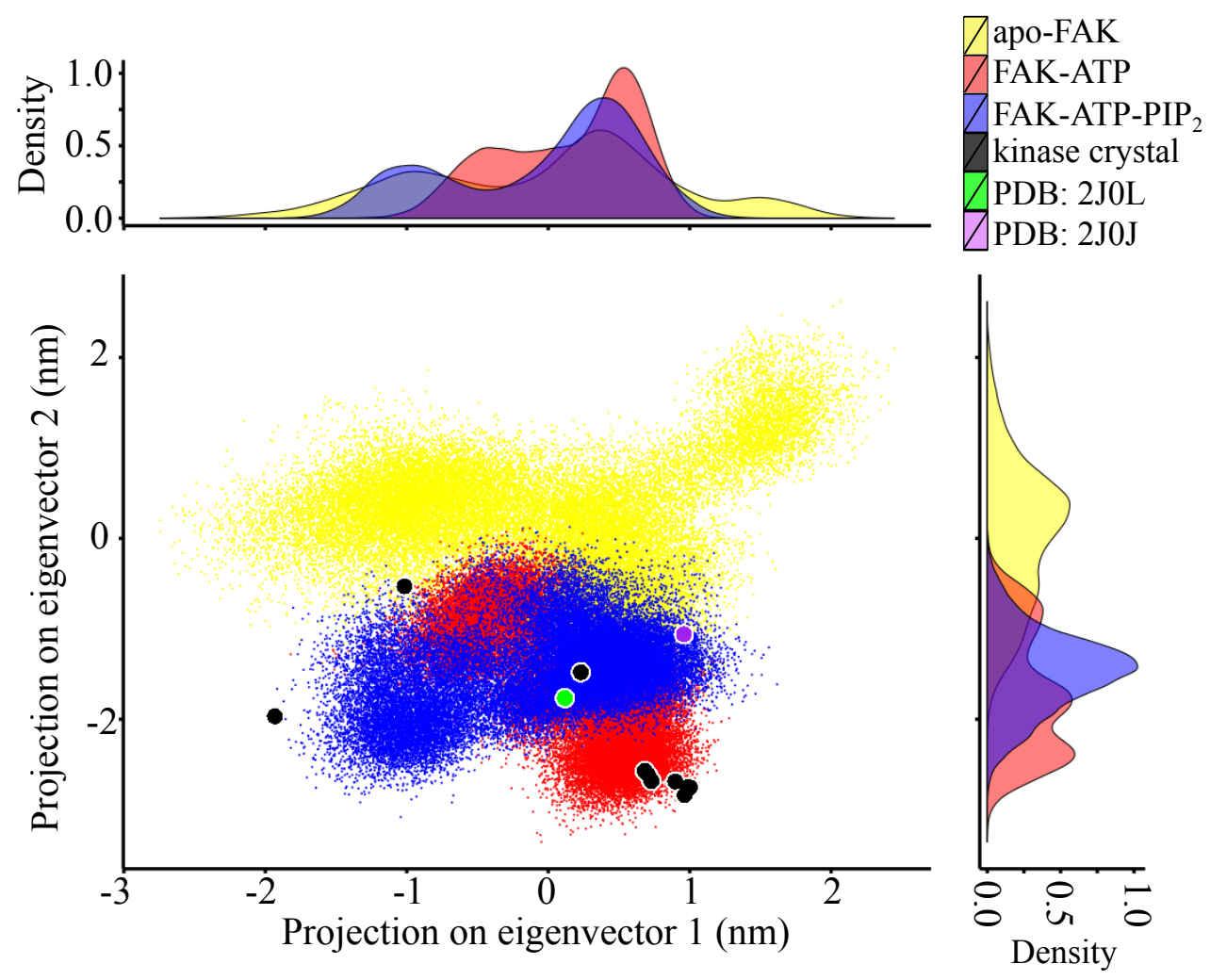

Figure 3.9 Conformational changes upon ATP/PIP 2 binding measured by principal component analysis (PCA). a) apo-FAK (yellow), FK-ATP (red), FK-ATP-PIP 2 (blue) during MD simulations, and crystal structures of the apo-FERM-kinase (violet, 2J0J), the ANP-bound FAK kinase (green, 2JOL), and apo-kinase domains (black) were projected on the first two eigenvectors obtained from a PCA of the fluctuations of apo-FAK $\mathrm{C}_{\alpha}$ atoms. Distributions of the three states along the two apo eigenvectors are also shown. 


\subsection{ATP and PIP2 induced allostery of FAK}

In the MD simulations, large-scale conformational changes in the FAK kinase domain and at the FERM-kinase interface could be observed. The changes were induced by ATP and $\mathrm{PIP}_{2}$ binding. These two opposite effects of ATP and $\mathrm{PIP}_{2}$ seem to be related, although their binding sites are located far away ( $4 \mathrm{~nm}$ and more) from each other, and also far away (1.5 $\mathrm{nm}$ and more) from the N/F1 interface and the auto-phosphorylation site. This leads to the question how these two ligands induce conformational changes in the protein at such distant sites. In order to answer this question, the molecular nature of the allosteric pathway connecting the involved sites was studied. To address this issue, we performed Force Distribution Analysis (FDA) of the ligand-protein complexes. FDA is a very sensitive technique, which measures changes in internal pair-wise forces induced by external perturbations such as ligand binding (see Section 2.3). Matrices of inter-residue forces were averaged over all trajectories for each state (apo-FK, FK-ATP, FK-ATP-PIP 2 ).

First, the allosteric effect of ATP, which induced FERM-kinase domain closure observed in previous experiment and in MD simulations, was studied. By comparing the inter-residue forces in apo-FK and FK-ATP, the residue pairs, with force differences between the two state beyond a certain cutoff, were identified and connected, and a force transduction network was generated. To reduce the noise, only connected graphs with more than 5 residues is displayed as a vertex [90]. Also, the two termini of the protein were excluded from the analysis as forces in these fluctuating regions are particularly noisy. When considering a high force cut-off ( $500 \mathrm{pN})$, the determined force network spreads from the ATP binding site through the activation loop, to the FERM F1 domain (Figure 3.10a). Apparently, ATP and $\mathrm{Mg}^{2+}$ act as an electrostatic "lock" by interacting with the adjacent charged residues, This leads to a restriction of inter-lobe fluctuations as observed in RMSFs measurement (Figure 3.7) and closure between the N- and C-lobe within the kinase domain as observed in PCA (Figure 3.9). With decreasing force cutoff (Figure 3.10b-c), the force transduction network expands to the $\mathrm{PIP}_{2}$ binding site in the FERM F2 lobe until almost covering the whole FERM-kinase domain interface, resulting in a destabilization of the helices within the kinase C-lobe at F2/C-lobe interface (Figure 3.7), though without a dissociation between the FERM and kinase domains (Figure 3.4). 
a)
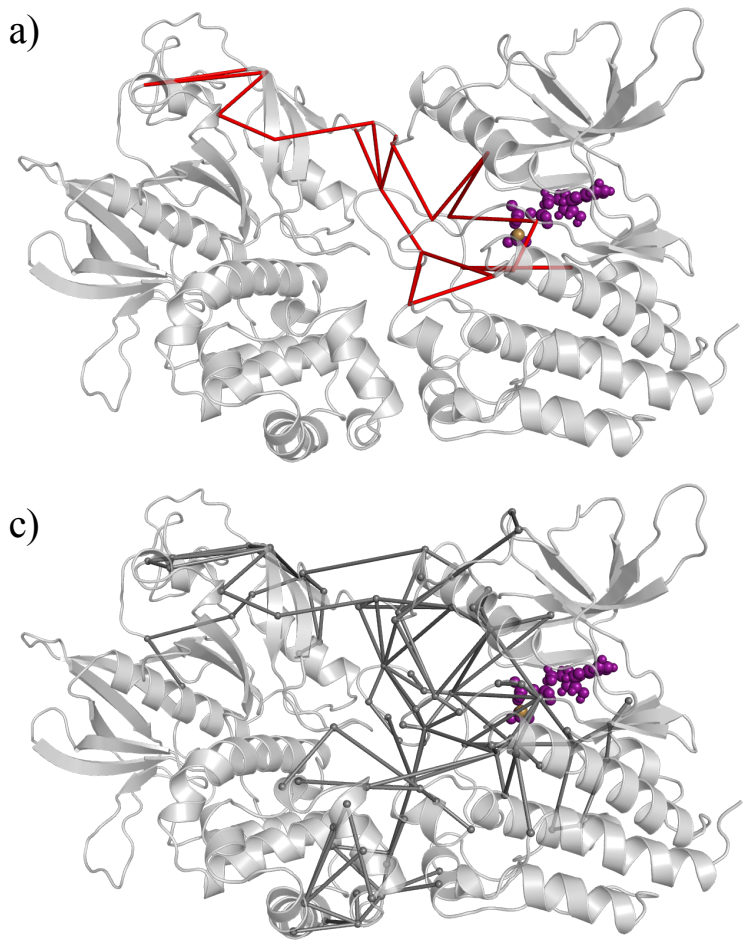

b)

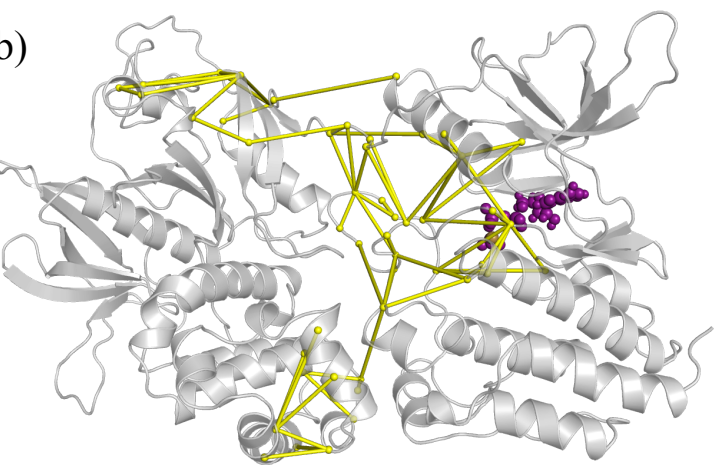

d)

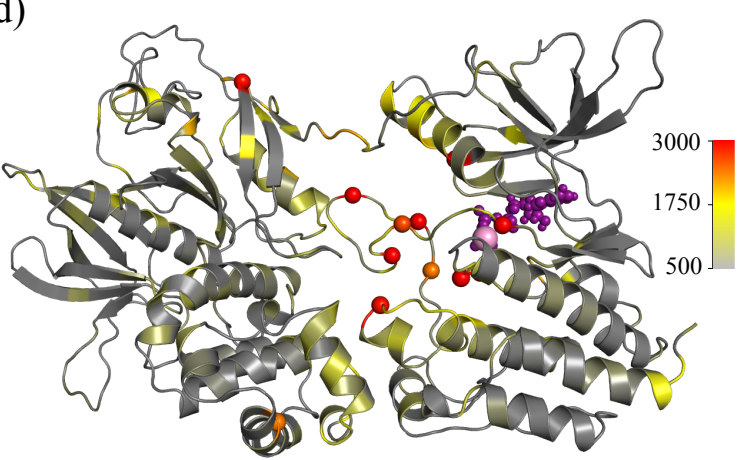

Figure 3.10 Force distribution upon ATP binding. (a-c) The network of force differences between apo-FAK and FK-ATP state simulations is shown as sticks. Only the networks of forces connecting more than 5 residues are shown. ATP (purple) is shown at a representative position, as observed during the simulations. The different colors of sticks indicate different force cutoffs, namely at a) $500 \mathrm{pN}$. b) $300 \mathrm{pN}$ and c) $200 \mathrm{pN}$. d) Difference of punctual stress, the sum of force differences acting on a given residue. Residues with stresses $>3000 \mathrm{pN}$ are shown as spheres. 

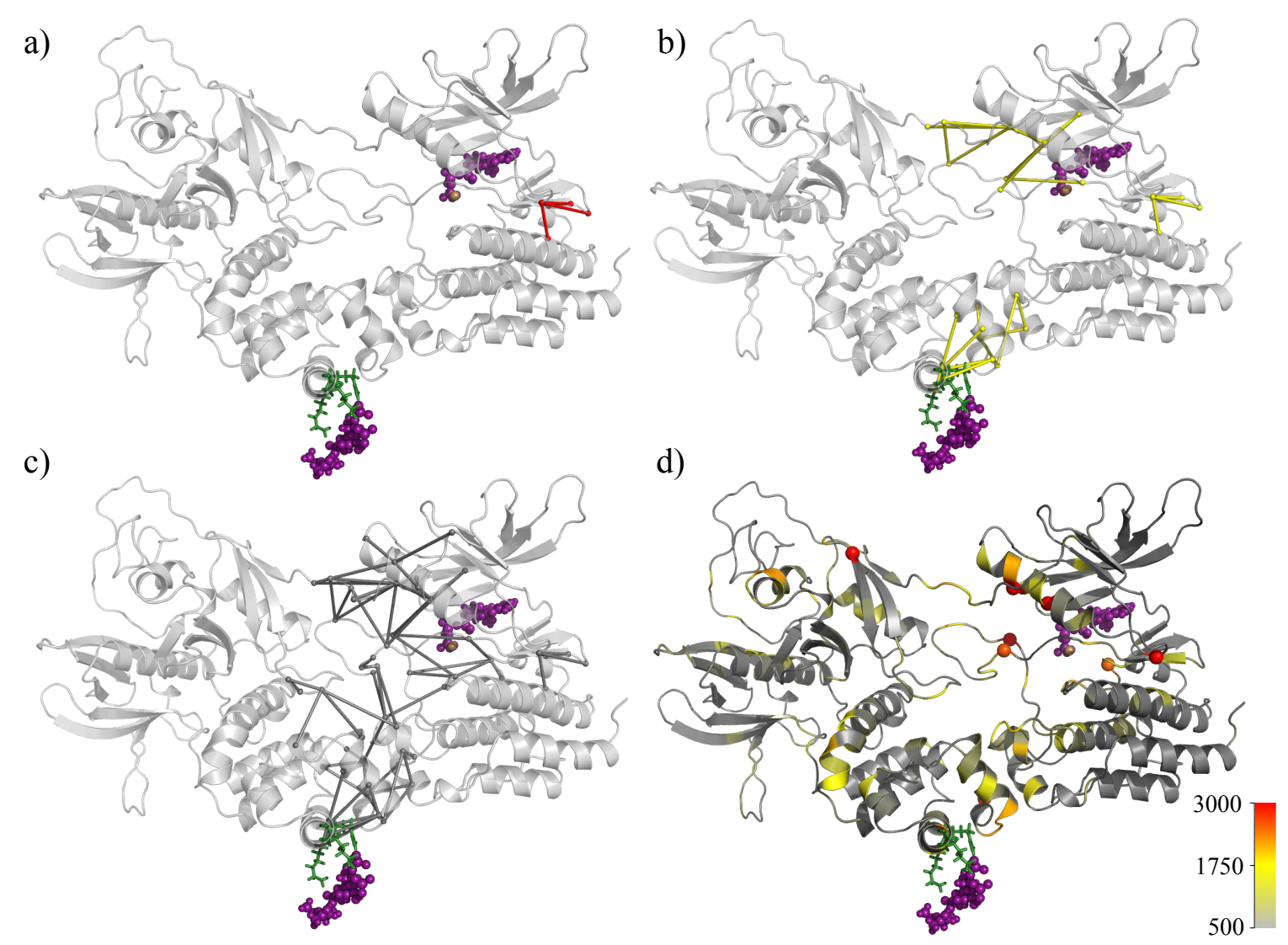

Figure 3.11 Force distribution upon $\mathrm{PIP}_{2}$ binding. (a-c) The network of force differences between FK-ATP and FK-ATP-PIP 2 state simulations is shown as sticks. Only the networks of forces connecting more than 5 residues are shown. ATP and PIP $_{2}$ (purple) are shown at representative position, as observed during the simulations. The different colors of sticks indicate different force cutoffs, namely at a) $350 \mathrm{pN}$, b) $300 \mathrm{pN}$ and c) $200 \mathrm{pN}$. d) Difference of punctual stress, the sum of force differences acting on a given residue. Residues with stresses $>3000 \mathrm{pN}$ are shown as spheres. 

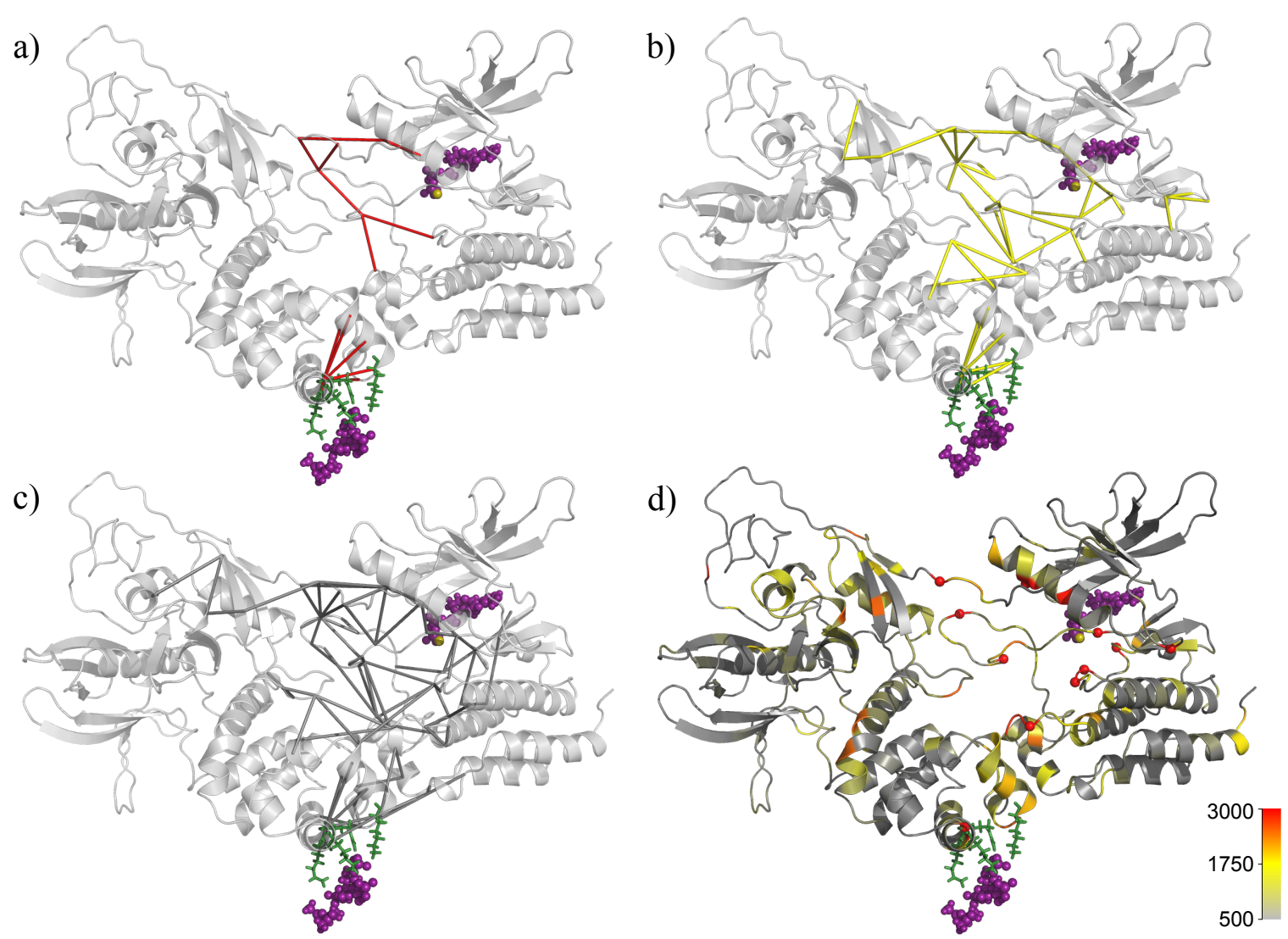

Figure 3.12 Force distribution upon ATP and PIP 2 binding. (a-c) The network of force differences between apo-FAK and FK-ATP-PIP 2 state simulations is shown as sticks. Only the networks of forces connecting more than 5 residues are shown. ATP and $\mathrm{PIP}_{2}$ (purple) are shown at representative positions, as observed during the simulations. The different colors of sticks indicate different force cutoffs, namely at a) $500 \mathrm{pN}$. b) 300 pN. c) 200 pN. d) Difference of punctual stress, the sum of force differences acting on a given residue. Residues with stresses $>3000 \mathrm{pN}$ are shown as spheres. 
Next, the inter-residue forces in FK-ATP and FK-ATP-PIP 2 were compared to understand the reverse allosteric effect of additional $\mathrm{PIP}_{2}$ binding on the inter-lobe closure within the kinase domain. This allosteric change resulted in a pronounced increase in the distance between the FERM F1 lobe and the kinase N-lobe. This is even more eyestriking than the ATP dependent allostery given the large distance of this region to the $\mathrm{PIP}_{2}$ binding site at F2. According to the force network depicted in Figure 3.11b-c, additional $\mathrm{PIP}_{2}$ binding to ATP-bound FAK results in a spatially connected force network, which spans the F2/C-lobe interface and the ATP binding site all the way up to the linker. The $\mathrm{PIP}_{2}$ head group is very negatively charged. The total charge on the whole molecule varies from -5 to -4 , depending on the $\mathrm{PIP}_{2}$ protonation state [85]. Thus, binding of $\mathrm{PIP}_{2}$ to the basic patch in the FERM domain causes substantial changes in the protein electrostatic potential right at the F2/Clobe interface, such that $\mathrm{PIP}_{2}$ binding indirectly adjusts the salt-bridge interactions in this region [41]. These structural adoptions then further distribute into the kinase N-lobe and adjacent linker, according to our FDA.

The network induced by the binding of $\mathrm{PIP}_{2}$ to ATP-bound FAK was detected at a force cut-off of $200 \mathrm{pN}$, which is much lower than the forces induced by ATP binding. A possible reason is that $\mathrm{PIP}_{2}$ resides at the well-structured FERM domain, in which both force transmission and structural changes are much less pronounced than in the susceptible kinase domain to which ATP binds. Indeed, with increasing force cutoff, the force network shrunk from the whole FERM-kinase interface to the dynamic kinase inter-lobe region domain $3.11 a$, a site overlapping with the hinge of the opening-closure motion. This indicates that the $\mathrm{PIP}_{2}$ binding remotely regulates the opening of the kinase domain without inducing large conformational changes within the F2-lobe.

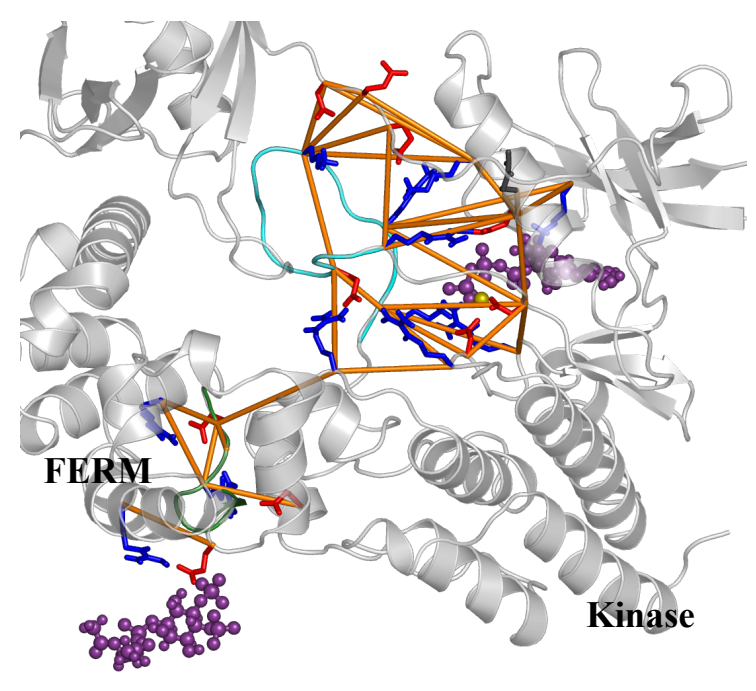

Figure 3.13 The force network (orange) connects residue pairs with a change $>250 \mathrm{pN}$ upon ATP binding and a reverse change of $>250 \mathrm{pN}$ upon $\mathrm{PIP}_{2}$ binding. (Marked red in Suppl. Table 1). Positively (blue) and negatively charged residues $($ red $)$ in this network are shown as sticks. The activation loop (residue 569-584, dark green) and the loop 181-189 (cyan) are shown as cartoon.

Since both computational results and 
experimental results reported to date support the finding of the opposite allosteric effect of ATP and PIP 2 on the FERM/kinase interface, it is not surprising to observe two largely overlapping force networks upon ATP and $\mathrm{PIP}_{2}$ binding, consisting of similar residue pairs (Figure 3.10 and Figure 3.11). In order to find a possible explanation for the distinct structural closure and opening motion induced by ATP and $\mathrm{PIP}_{2}$ respectively, the inter-residue forces of the residue pairs involved in these two allosteric networks are listed in Table 3.1 and analyzed according to their changes in the three states. If the inter-residue force of one residue pair is change by ATP binding $(>250 \mathrm{pN})$ and then rescued by PIP $_{2}$ binding (also $>250 \mathrm{pN}$ ), this residue pair is marked red in the table. In Figure 3.13, these residue pairs with significant reverse force changes are shown as sticks. This pathway includes all residues, which might regulate the motions giving rise to the FRET signal changes upon ligand binding. As can be seen in this pathway, one or both residues in each highlighted pair is either located in the activation loop (residues 569-584) or in the loop (residues 180-190) at the F2/C-lobe interface. The former is known for its pivotal role in allosteric regulation of kinases. The latter has been detected to be disordered in the FAK FERM domain containing a basic patch mutated by charge-neutralizing residues that is meant to mimic $\mathrm{PIP}_{2}$ binding [41]. It is remarkable that all except one of the interactions within the resulted pathway are salt-bridges that reversibly swap partners. This relative motions of the residues identified in the force pathway, which are based on electrostatic interactions, seem to be the molecular basis for the dramatic conformational changes within kinase domain and between the kinase domain and the auto-inhibitory FERM domain. 
Table 3.1 Average residue pairwise forces within connected networks upon ATP and PIP $_{2}$ binding as pointed out by FDA and as shown in Figures 3.10 and 3.11. Residue pairs with a change $>$ $250 \mathrm{pN}$ upon ATP binding and a reverse change of $>250 \mathrm{pN}$ upon $\mathrm{PIP}_{2}$ binding are marked in red and highlighted in Figure 3.13. All of them except one are salt-bridges.

\begin{tabular}{|c|c|c|c|c|c|c|c|c|c|}
\hline \multicolumn{2}{|c|}{$\begin{array}{l}\text { Residue } \\
\text { pair }\end{array}$} & \multirow{2}{*}{$\begin{array}{l}\begin{array}{l}\text { APO } \\
(\mathrm{pN})\end{array} \\
-559\end{array}$} & \multirow{2}{*}{$\begin{array}{l}\text { ATP } \\
(\mathrm{pN}) \\
-937\end{array}$} & \multirow{2}{*}{$\begin{array}{c}\begin{array}{c}\text { ATP-PIP } \\
(\mathrm{pN})\end{array} \\
-403\end{array}$} & \multicolumn{2}{|c|}{$\begin{array}{c}\text { Residue } \\
\text { pair }\end{array}$} & \multirow{2}{*}{$\begin{array}{l}\begin{array}{l}\text { APO } \\
(\mathrm{pN})\end{array} \\
-104\end{array}$} & \multirow{2}{*}{$\begin{array}{l}\text { ATP } \\
(\mathrm{pN}) \\
-447\end{array}$} & \multirow{2}{*}{$\begin{array}{c}\begin{array}{c}\text { ATP-PIP } \\
(\mathrm{pN})\end{array} \\
-195\end{array}$} \\
\hline R35 & D60 & & & & E404 & R569 & & & \\
\hline R35 & R108 & 637 & 1014 & 796 & K454 & E471 & -538 & -941 & -630 \\
\hline R35 & E109 & -7 & -988 & -114 & K454 & K581 & 1.473 & 322 & 3 \\
\hline R35 & E112 & -829 & -1179 & -1082 & K467 & E403 & -258 & -489 & -14 \\
\hline R35 & E118 & -885 & -8 & -330 & K467 & $\mathrm{E} 471$ & -108 & -418 & -757 \\
\hline R35 & D395 & 0 & -351 & -105 & K467 & К578 & 266 & 554 & 125 \\
\hline K38 & E403 & -785 & -26 & -9 & E471 & Q470 & 270 & 582 & 378 \\
\hline R57 & D462 & -635 & -50 & 0 & E471 & D564 & 16 & 413 & 378 \\
\hline D60 & R108 & -552 & -234 & -668 & E471 & R569 & -952 & -74 & -682 \\
\hline D60 & E109 & 15 & 521 & 232 & E471 & K581 & 0 & -845 & 2 \\
\hline D60 & D395 & 1 & 400 & 245 & $\mathrm{H} 482$ & Q529 & 91 & 49 & 773 \\
\hline E109 & E112 & 130 & 458 & 168 & H482 & D558 & 32 & -62 & 481 \\
\hline E118 & K38 & -185 & -1272 & -1144 & H482 & C559 & 64 & 6 & 1084 \\
\hline R177 & E182 & -174 & -620 & -410 & H482 & V560 & -124 & -67 & 1352 \\
\hline R177 & R184 & 120 & 466 & 173 & R545 & E572 & -126 & -1038 & -1094 \\
\hline E182 & M183 & 636 & 309 & 300 & D546 & K583 & -779 & -1675 & -1158 \\
\hline E182 & R184 & -649 & -943 & -546 & D564 & H544 & -80 & 256 & 194 \\
\hline E182 & R597 & -502 & -176 & -397 & D564 & D546 & 677 & 2231 & 2381 \\
\hline R184 & K190 & 164 & 10 & 319 & D564 & R550 & -986 & -528 & -324 \\
\hline R184 & E636 & -367 & -76 & -693 & D564 & F565 & 391 & -37 & 111 \\
\hline E189 & R221 & -6 & -546 & -650 & D564 & K583 & -222 & -557 & -412 \\
\hline E189 & K222 & -109 & -419 & -294 & R569 & D405 & -202 & -725 & -765 \\
\hline E189 & R229 & -915 & -477 & -675 & E572 & Y570 & -289 & 135 & 105 \\
\hline K190 & E592 & -799 & -563 & -37 & E572 & D573 & 400 & 725 & 681 \\
\hline K191 & K218 & 155 & 143 & 475 & K578 & E404 & -4 & -669 & -905 \\
\hline K191 & R221 & 339 & 455 & 858 & K578 & E572 & -1215 & 6 & 1 \\
\hline R221 & E195 & -1109 & -505 & -151 & K578 & Y576 & 120 & 583 & 604 \\
\hline R221 & E198 & -1178 & -479 & -168 & K578 & S580 & 361 & -32 & -1 \\
\hline R221 & K199 & 608 & 174 & 73 & L580 & A579 & -190 & 178 & 112 \\
\hline R221 & K 222 & 488 & 671 & 1277 & K581 & D564 & -8 & -542 & -2 \\
\hline D395 & R57 & 0 & -405 & -393 & K583 & R550 & 492 & 1166 & 475 \\
\hline D395 & D63 & 532 & 119 & 22 & R597 & R545 & -9 & 540 & 316 \\
\hline D402 & K467 & -1068 & -620 & -44 & R597 & E572 & -32 & -874 & -568 \\
\hline D402 & K578 & -136 & -1193 & -1248 & R597 & D573 & -1025 & -439 & -663 \\
\hline E403 & D402 & 609 & 916 & 664 & R597 & T575 & 309 & -3 & -3 \\
\hline E403 & K578 & -3 & -723 & -358 & E636 & E592 & 458 & 365 & 61 \\
\hline
\end{tabular}




\subsection{Discussion and Conclusions}

We used molecular simulations to study the allosteric effects of ATP and PIP 2 on FK-FAK. The computational results can complement the experimental findings [41], and reveal the underlying molecular details of PIP $_{2}$-mediated FAK partial activation. Using MD simulation for the three different protein systems (apo-FK, FK-ATP, FK-ATP-PIP 2 ), we observed the same low-frequency motion of the partial opening of FK-FAK as detected by previous FRET measurement. Further analysis, namely PCA and FDA, allowed to identify and to describe the role of ATP and PIP $_{2}$ in this dynamic allostery.

The highly charged ATP/ $\mathrm{Mg}^{2+}$ acts as a hub for electrostatic interactions with the charged residues in the active site of the kinase domain and, thus, restricts the inter-lobe motion between the kinase $\mathrm{N}$ - and $\mathrm{C}$-lobe, including twisting and opening. The additional binding of the highly negatively charged $\mathrm{PIP}_{2}$ at the basic patch in the FERM F2-lobe changes the electrostatic interactions between the basic patch (residues 216-222) and its adjacent $\alpha$ helix (residues 181-198) at the F2/C-lobe interface. This leads to a domino-like cascade of changes in salt-bridges from F2/C-lobe interface through the kinase domain to the F1/Nlobe interface and to a sequential decrease of the auto-inhibitory interaction between the FERM and kinase domains.

Based on these results, we can conclude that the allosteric effect of $\mathrm{PIP}_{2}$ is mainly the change of the electrostatic nature of the FERM F2-lobe leading to a restriction of F2-lobe fluctuations and a consequent rearrangement of the interaction the between FERM F2 and kinase C-lobe. We believe that the other basic patch interaction partners, such as C-Met [91] or the FAT domain, might affect FAK dynamic allostery in a similar way.

Besides the molecular description of PIP $_{2}$ mediated FAK partial activation, the computational results described in this chapter allowed to identify important residues involved in this allostery to predict mutations, which might be critical for the FAK activation. By considering the position of each highlighted residues in Table 3.1 and the number of its connections with the nearby residues, we suggest to test the mutations at R184 and R597 of ATP and $\mathrm{PIP}_{2}$-dependent allostery.

As proposed in the the complex model of sequential FAK activation described in a previous study [41], we were able to observe the opening between FAK FERM F1- and kinase $\mathrm{N}$-lobe. Y397 is located in the area of the linker, which formed a $\beta$-sheet conformation with 
the F1-lobe. The experimental study demonstrates that $\mathrm{PIP}_{2}$ binding leads to the dissociation of the linker from the F1-lobe and, thus, enhances the auto-phosphorylation of Y397 according to the experimental phosphorylation assays. However, we could not detect any significant change in structure or dynamics of Y397 in the relaxed FERM/kinase conformation, which would explain the $\mathrm{PIP}_{2}$-dependent regulation of FAK. The reason for that might be the limited sub-microsecond time scale of the MD simulations that was reached. As described in the previous studies on other proteins [92, 93], FDA is a versatile method able to reveal signal propagation in the protein by monitoring internal forces even upon minor conformational distortions, which initiate the large scale and ofter show conformational transitions.

Since the activation loop is critically involved in the detected allosteric pathway, we calculated its fluctuations. As shown in Figure 3.14, ATP binding strongly reduced the flexibility of most residues in the activation loop, including the major phosphorylation site of Y576/577. However, $\mathrm{PIP}_{2}$ binding released this constraint. That is to say that the relaxed FAK FERM/kinase conformation upon $\mathrm{PIP}_{2}$ binding can not only enhance the efficiency of FAK autophosphorylation, but can also lead to the increase in Src phosphorylation of Y576/577 as observed in the experiment [41] by in-

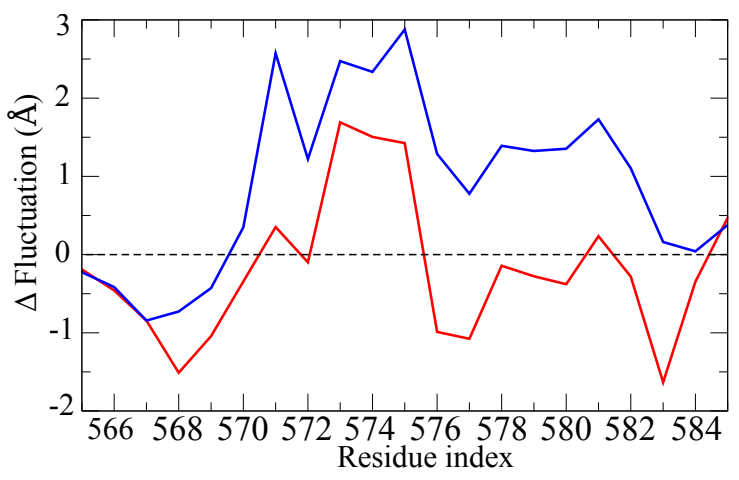

Figure 3.14 Ligand-induced changes in the activation loop at the FERM-kinase interface, where the major phosphorylation site Y576/577 is located. Differences in root-mean-square (RMS) fluctuations to the apo-state as reference are shown in blue for FK-ATP and in red for FK-ATP-PIP 2 . creasing the flexibility of the activation loop.

In agreement with the experimental data [41], none of the simulations showed full opening of FK-FAK. In other words, $\mathrm{PIP}_{2}$ alone is not sufficient to induce catalytic turnover of FAK which is required for Src recruitment for subsequent Y576/577 phosphorylation. Therefore, an additional stimulus, such as external mechanical force, might also contribute to FAK activation. For several focal adhesion proteins, it has been shown that stretching forces can regulate focal adhesion maturation and signaling [13, 94, 95]. Moreover, several studies described FAK as a key tension sensor in focal adhesions [31, 96, 97]. FAK's FAT domain binds to actin filaments via paxillin [98], and FAK's FERM domain binds to the 
membrane via $\mathrm{PIP}_{2}$ [41, 42]. It is therefore feasible that stretching forces at focal adhesion sites propagate through these attachment points to FAK and enhance FAK activation. The calculations performed in the framework of this chapter have shown that $\mathrm{PIP}_{2}$ and ATP binding are important first steps in FAK allosteric control. In the next chapter, we shall describe results on the force-regulated activation of FAK. 



\section{CHAPTER 4}

\section{FORCE-INDUCED ACTIVATION OF FOCAL ADHESION KINASE}

This chapter presents the results of out investigation on how an external force can release the major phosphorylation site Y576/577 from the auto-inhibitory FERM domain of FAK. The extensive FPMD simulations (Section 2.1.3) of the FAK FERM-kinase fragment under various conditions were carried out to test if a mechano-activation of FAK at FAs is a plausible scenario. Then, the obtained force-dependent kinetic parameters were employed in a biochemical reaction model to study the role of FAK as a mechano-enzyme in the regulation of the GTPase Ras.

\subsection{Introduction}

The dynamic allostery of FK-FAK upon ATP and PIP 2 binding has been discussed in Chapter 3. Both experimental [36] and computational observations show that the binding of $\mathrm{PIP}_{2}$ to ATP-bound FAK can induce partial opening of FK-FAK, resulting in an increase in FAK auto-phosphorylation. However, PIP $_{2}$ cannot induce FAK catalytic turnover, which requires a fully opened state of the FAK FERM-kinase fragment. Thus, FAK activation requires an additional biochemical stimulus or, potentially, external force, to fully release the auto-inhibitory FERM domain from the kinase domain. 
The function of FAK as a cellular mechano-sensor involved in the regulation of multiple signaling pathway has been emphasized in Chapter 1. On this basis, we suggest external force as an additional stimulus for FAK activation. To test our hypothesis, the first question to be answered is how mechanical force propagates to FAK. PIP 2 appears to be an excellent candidate for propagating force onto FAK. PIP 2 is an ubiquitous second messenger enriched in the innerleaflet of the plasma membrane and concentrated at FAs. Experiments have shown that $\mathrm{PIP}_{2}$ regulates the interaction of cytoskeletal proteins with the membrane [99-101]. Although the global concentration of $\mathrm{PIP}_{2}$ in the cell membrane is only $1 \%$ [102], the local accumulation of $\mathrm{PIP}_{2}$ at the site of docked vesicles exceeds the cellular $\mathrm{PIP}_{2}$ concentration [103]. Recent experiments also showed that $\mathrm{PIP}_{2}$ clusters can be induced by $\mathrm{PIP}_{2}$-protein interactions $[102,104]$ or by simply adding calcium or other divalent ions $[105,106]$. In addition, local $\mathrm{Ca}^{2+}$ concentration elevations have been suggested to be able to increase the residency of FAK at FAs [107]. All these findings indicate that the aggregation of $\mathrm{PIP}_{2}$ at FAs might be critical for in the FAK activation.

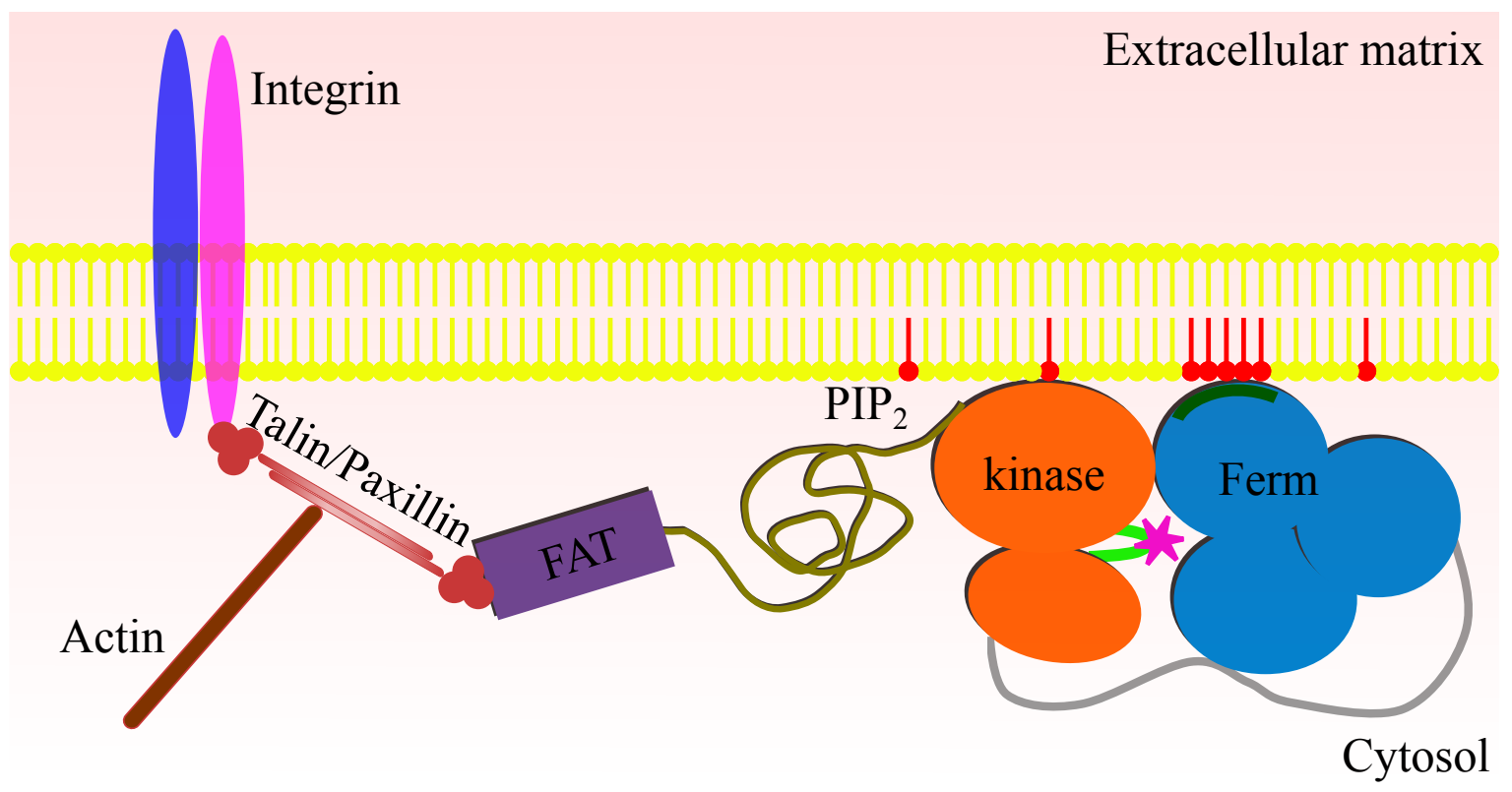

Figure 4.1 Mechanical model of FAK activation represents how FAK connects membrane and cytoskeleton. The basic patch (dark green) in the FERM domain (blue) binds to the membrane (light yellow) through direct interaction with $\mathrm{PIP}_{2}$ cluster (red). The major phosphorylation site, Y576/577 (purple star), located at the activation loop (light green), is auto-inhibited by the FERM domain in equilibrium. Through a disordered proline rich region, the kinase domain (orange) is connected to the FAT domain (violet). FAT domain binds indirectly to integrin and actins via talin.

The following aspects support the notion of FAK as a mechano-enzyme. First, FAK 
is tethered between the PIP $_{2}$-enriched membrane and the cytoskeleton, likely acting as a force-carrying link in FAs. Second, the FERM-kinase structure suggests itself as a mechanoresponsive scaffold, in which force could specifically detach the auto-inhibitory FERM domain. Third, FAK would be the first mechano-enzyme of FAs, allowing a direct transduction of a mechanical signal into an enzymatic reaction and downstream events, which would yield a mechanistic explanation of FAK's mechano-sensing role [30]. Indeed, two analogous cases of mechanically activated enzymes have been previously identified, both of which are kinases and feature force-induced activation by removal of an auto-inhibitory domain, namely titin and twitchin kinase in muscle [47, 108-110].

To test the hypothesis of FAK as a force-sensor, extensive Force-Probe Molecular Dynamics (FPMD) simulations of the structurally known FK-FAK have be carried out under various conditions. Force can propagate onto FAK from the membrane through its PIP $_{2}$ binding site of FERM and from the cytoskeleton-targeting FAT domain through the prolinerich linker to the kinase domain as illustrated in Figure 4.1. We find this mechanotransduction pathway to specifically open the hydrophobic FERM-kinase interface, leading to FAK activation prior to the unfolding of the kinase domain. Given the mediocre stability of the largely $\alpha$-helical kinase and FERM domains, this is remarkable. The enforced activation is robust with regard to a large range of pulling velocities, but sensitive to the pulling direction. Extrapolation to physiological time scales and employment of a biochemical reaction model allowed to quantitatively infer the FAK-mediated mechanotransduction into the Ras pathway. Our force-induced activation pathway suggests a direct mechano-enzymatic function of FAK in FAs.

\subsection{Materials and Methods}

\subsubsection{FPMD simulations}

Two systems were subjected to force during FPMD simulations: (1) the FAK fragment consisting of the FERM and kinase domains as a single protein, and (2) the same fragment in complex with a membrane. The initial structure of the FAK fragment (sequence region: 35-686) was taken from its crystal structure [36] (PDB code: 2J0J), completed by MODBASE [81], and equilibrated for $200 \mathrm{~ns}$ as described in the previous equilibrium MD simulations (see Chapter 3). 
In the FPMD simulations of the FAK in absence of membrane, the C-terminus of the kinase domain and the FERM basic patch were pulled away from each other at a constant pulling speed $V$ (Figure 2.5). The pulled elements were subjected to a time-dependent harmonic force, by attaching them to moving harmonic springs with constant velocities. The applied force reads

$$
F_{i}(t)=-k\left[z_{i}(t)-V_{i} t\right]
$$

where the elastic constant $k$ is equal to $500 \mathrm{~kJ} / \mathrm{mol}, z_{i}(t)$ is the position of the center of mass of the kinase $\mathrm{C}$-terminus $(i=1)$ and the FERM basic patch $(i=2)$ along the pulling coordinate $z$, and $V_{i}$ is the velocity of the moving springs $\left(V_{1}=-V / 2\right.$ and $\left.V_{2}=V / 2\right) . V=0.006$, $0.014,0.03,0.05,0.07,0.08,0.09,0.1,0.16,0.24,0.3$, and $0.5 \mathrm{~nm} / \mathrm{ns}$ were used.

FPMD simulations were carried out using GROMACS 4.0.5 [61]. The protein was accommodated at the center of a water box of $18 \times 10 \times 10 \mathrm{~nm}^{3}$ dimension. The protein OPLS all-atom force field [62] and the the TIP4P water model [57] were used for the simulations. All bonds were constrained using the LINCS algorithm [53]. The temperature was kept constant at $\mathrm{T}=300 \mathrm{~K}$ by using the velocity rescaling thermostat [111], with a coupling time of $0.1 \mathrm{ps}$. The pressure was kept constant at 1 bar by coupling the system to a Parrinello-Rahman barostat [87] with a coupling time of 0.1 ps. The electrostatic interactions were treated with the Particle Mesh Ewald [88] method using a grid spacing of 0.12 $\mathrm{nm}$ with a cubic interpolation. Short-range interactions were modeled through a LennardJones potential. An integration time step of $2 \mathrm{fs}$ was used. After an energy minimization of 10000 steps using the steepest descent algorithm, the whole system was equilibrated by a $2 \mathrm{~ns}$ MD simulation with harmonic constraints on the protein and membrane heavy atoms with a force constant of $k=1000 \mathrm{kJmol}^{-1}$.

In the simulations including the membrane, the FERM domain was anchored via $\mathrm{PIP}_{2}$ lipids to a palmitoyloleoylphosphatidylethanolamine (POPE) lipid bilayer built by the CharmmGUI [112] as shown in Figure 4.2. The ATP/ $\mathrm{Mg}^{2+}$ were placed at the kinase domain in the orientation close to the one observed for the ANP in the crystal structure (PDB: 2J0L) [36]. The $\mathrm{PIP}_{2}$ head group $\left(\mathrm{C} 2-\mathrm{PIP}_{2}\right)$ was docked to the FERM basic patch $\left({ }^{216} \mathrm{KAKTLR}^{221}\right)$ [41, 42], using the UCSF DOCK6.5 suite [86] (see Section 2.2). The resulting structure was equilibrated during $150 \mathrm{~ns}$ of MD simulation. Three different concentrations of $\mathrm{PIP}_{2}$ lipids $(1 \%, 10 \%$, and $15 \%)$ in the lipid membrane were tested. The resulting membranes containing $\mathrm{PIP}_{2}$ and POPE lipids were equilibrated during $100 \mathrm{~ns}$ of MD simulations. The head group of one of the $\mathrm{PIP}_{2}$ lipids, embedded in the bilayer, was then replaced by the FAK 
$\mathrm{PIP}_{2}$-head-group complex, in order to obtain a FAK-PIP 2 -membrane complex. Such obtained complex was immersed in a triclinic water box. $0.1 \mathrm{M} \mathrm{NaCl}$ was added to the system and the charge of the unit was neutralized by adding $\mathrm{Ca}^{2+}$ ions. The complex was equilibrated by $150 \mathrm{~ns}$ of MD simulations, and the last snapshot was considered as the starting conformation for the FPMD simulations.

FPMD simulations were carried out using GROMACS 4.5.5 [113]. The protein-membrane complex was accommodated at the center of a water box of $15 \times 10 \times 17 \mathrm{~nm}^{3}$ dimension . The modified CHARMM36 force field containing parameters of $\mathrm{PIP}_{2}$ lipid [114] and the TIPS3P water model [115], was used for the simulations of the protein-membrane complex. Bonds involving hydrogen atoms were constrained using the LINCS algorithm [53]. The temperature was kept constant at $\mathrm{T}=300 \mathrm{~K}$ by using the velocity rescaling thermostat [111], with a coupling time of $0.1 \mathrm{ps}$. The pressure was kept constant at 1 bar by coupling the system to a Parrinello-Rahman barostat [87] with a coupling time of $0.1 \mathrm{ps}$. The electrostatic interactions were treated with the Particle Mesh Ewald [88] method using a grid spacing of $0.15 \mathrm{~nm}$ with a cubic interpolation. Short-range interactions were modeled through a Lennard-Jones potential. An integration time step of 2 fs was used. After an energy minimization of 10000 steps using the steepest descent algorithm, the whole system were equilibrated by a $2 \mathrm{~ns}$ MD simulation with harmonic constraints on the protein and membrane heavy atoms with a force constant of $k=1000 \mathrm{kJmol}^{-1}$.

In the FAK-membrane complex simulations, the C-terminus of the Kinase domain was pulled away from the membrane, in vertical $\left(90^{\circ}\right)$ or diagonal $\left(45^{\circ}\right)$ direction to the membrane plane (Figure 4.2). The same harmonic forces were exerted on the pulled elements as in the FAK single protein simulation (Eq 4.1), but the kinase C-terminus was pulled at a speed of $V=0.03$ and $0.05 \mathrm{~m} / \mathrm{s}$, while the membrane was maintained at rest $\left(V_{2}=0\right)$. 


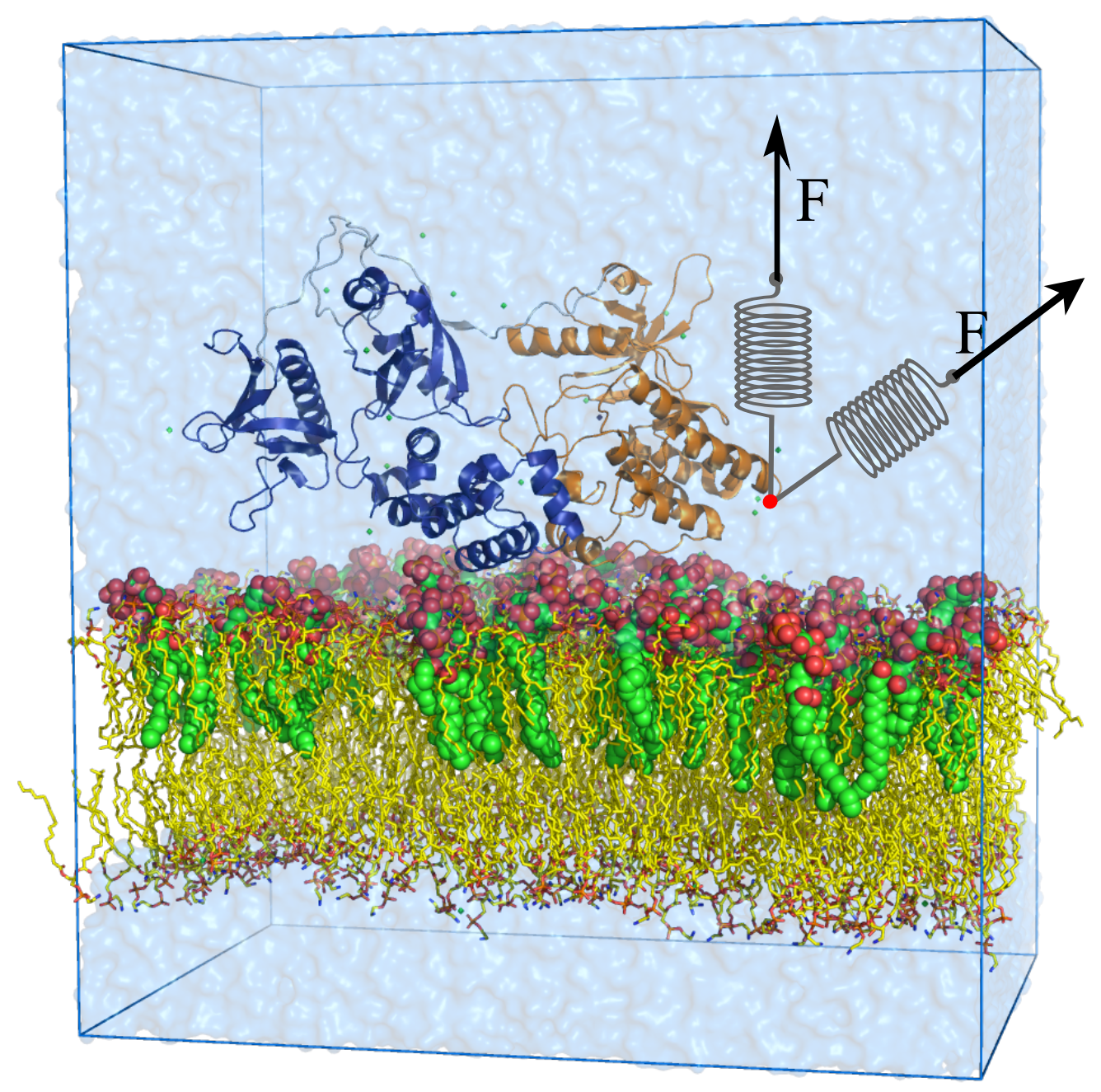

Figure 4.2 Simulation system of the FAK-membrane complex in a water box. FK-FAK (shown as cartoon) interacts with the POPE membrane (shown as sticks in yellow) via $\mathrm{PIP}_{2}$ (shown as spheres and colored according to atom types). The gray springs represent the harmonic potentials applied on the $\mathrm{C} \alpha$ atoms of the kinase $\mathrm{C}$-terminal residue (shown as red dot) in vertical $\left(90^{\circ}\right)$ and diagonal $\left(45^{\circ}\right)$ directions. 


\subsubsection{PIP2 aggregation in POPE membrane bilayer}

To detect the effect of $\mathrm{PIP}_{2}$ lipids on the force-induced FAK activation, different POPE membrane systems with $1 \%, 10 \%$ and $15 \% \mathrm{PIP}_{2}$ concentrations were set up and equilibrated using the CHARMM36 force field (details see Section 4.2.1). $\mathrm{PIP}_{2}$ lipids in the membrane are supposed to aggregate in the environment with $\mathrm{Ca}^{2+}$ by forming so-called lipid rafts [107]. To trace the aggregation or at least an interaction within $\mathrm{PIP}_{2}$ molecules in the membrane, the distances between phosphor and phosphor atoms and between phosphor and calcium atoms were calculated. If the P-P length was shorter than $0.6 \mathrm{~nm}$ or a P-Ca length was shorter than $0.4 \mathrm{~nm}$, the two atoms were considered to interact. By building a $N \times N$ matrix, where $N$ is equal to the total number of phosphor and calcium atoms, and assigning 1 to each interacting pair or 0 to each non-interacting pair, clustering within $\mathrm{P}$ and $\mathrm{Ca}$ atoms was determined. If a group of interacting atoms contained more than three phosphor atoms, it was defined as a cluster [116] of aggregated $\mathrm{PIP}_{2}$. The numbers of $\mathrm{PIP}_{2}$ clusters and aggregated $\mathrm{PIP}_{2}$ lipids were determined at each simulation step, which allowed us to trace the process of $\mathrm{PIP}_{2}$ aggregation. We note that due to the limited system size, clustering here refers to microclusters of only a few $\mathrm{PIP}_{2}$ molecules, in contrast to the larger rafts observed commonly in experiments.

\subsubsection{Fitting of rupture forces at varying loading rates}

The Dudko-Hummer-Szabo (DHS) [50] and the Hummer-Szabo (HS) [51] models were employed to fit the resulted rupture forces (see Section 2.1.3) as a function of the loading rate. At the intermediate loading-rate regime, where stochastic motions are relevant, the average of the rupture force $\langle F\rangle$ can be expressed in terms of the loading rate $L=k V$ ( $k$ is the spring elastic constant and $V$ the pulling speed) according to the DHS model [50]:

$$
\langle F\rangle=\frac{\Delta G}{v x}\left\{1-\left[\frac{1}{\beta \Delta G} \ln \frac{k_{0} e^{\beta \Delta G+\gamma}}{x \beta L}\right]^{v}\right\} .
$$

Here, $\Delta G, x$ and $v$ reflect the underlying free energy landscape governing the dissociation process: $x$ is the distance between the FK-FAK closed state and the transition state, $\Delta G$ represents the height of energy barrier, and $v=1 / 2$ corresponds to a cusp shape of the free energy surface $k_{0}$ is the dissociation rate at zero force; $\beta=1 / k_{B} T$, with $k_{B}$ the Boltzmann 
constant and $T$ the temperature, and $\gamma=0.5772 \ldots$ is the Euler-mascheroni constant.

If the pulling velocity was sufficiently large, and thereby the loading rate was much larger than the critical loading rate $\left(L_{c r i t} \equiv k_{0} e^{\beta \Delta G} /(\beta x)\right)$, the stochastic motions are irrelevant and $\langle F\rangle$ depends on $L$ according to the DHS model [50]:

$$
\langle F\rangle=\frac{\Delta G}{v x}+\frac{x^{2} L}{2 D \beta \Delta G}\left[1-\exp -\left(\frac{8 D \beta \Delta G^{2}}{x^{3} L}\right)^{1 / 2}\right],
$$

where, $D$, the diffusion coefficient, is given by $D=k_{0} \sqrt{2 \pi}(2 \beta \Delta G)^{-3 / 2} x^{2} \exp (\beta \Delta G)$, based on Kramer's theory [50]. Here it has been assumed that $2 \beta \Delta G / x^{2} \gg k$, i.e. the intrinsic spring constant of the free energy surface is larger than the elastic constant of the pulling spring.

The average rupture forces obtained from the FPMD simulations at different loading rates were fitted by using $\Delta G$ and $x$, and $L_{c r i t}$, as fitting parameters. Note that $k_{0}$ can be computed from these three parameters, from the definition of the critical loading rate. A Monte Carlo sampling was used to do the fitting. $\Delta G, x$ and $L_{c r i t}$ values were randomly chosen, with $\Delta G$ in the range from $25 k_{B} \mathrm{~T}$ to $60 k_{B} \mathrm{~T}, x$ from $0.2 \mathrm{~nm}$ to $1.8 \mathrm{~nm}$, and $L_{c r i t}$ from $1 \mathrm{e}-7 \mathrm{pN} / \mathrm{ps}$ to $5 \mathrm{pN} / \mathrm{ps}$ (Figure 4.16).

With these parameters, the rupture force $\left.\left\langle F_{\text {model }}\right)\right\rangle$ was computed for all loading rate values used in the FPMD simulations by using Equation 4.2 (for $L<=L_{c r i t}$ ) and Equation 4.3 (for $\left.L>L_{c r i t}\right)$. The root mean square deviation (RMSD) between predicted $\left(F_{\text {model }}\right)$ and the resulting $(F)$ rupture forces was given as

$$
R M S D=\sqrt{\frac{1}{N} \sum_{i=1}^{N}\left(F_{i}-F_{i, \text { model }}\right)^{2}}
$$

and used to score the predicted kinetic parameters. 


\subsection{Force induced exposure of the activation loop.}

In vitro, in absence of additional stimuli, no phosphorylation of the activation loop of FAK is observed, neither in the free form [36] nor when bound to $\mathrm{PIP}_{2}$ containing vesicles [41]. Based on previous studies, external force is suggested as an additional biochemical stimulus to expose the buried activation loop between FAK FERM and kinase domains. Thus, extensive FPMD simulations were carried out to examine the effect of tensile stress on the auto-inhibited FK-FAK conformation. FAK is tethered between the membrane and the cytoskeleton, resulting in force transmission from the membrane onto the basic patch of the FERM domain and from the paxillin/talin-interacting FAT do-

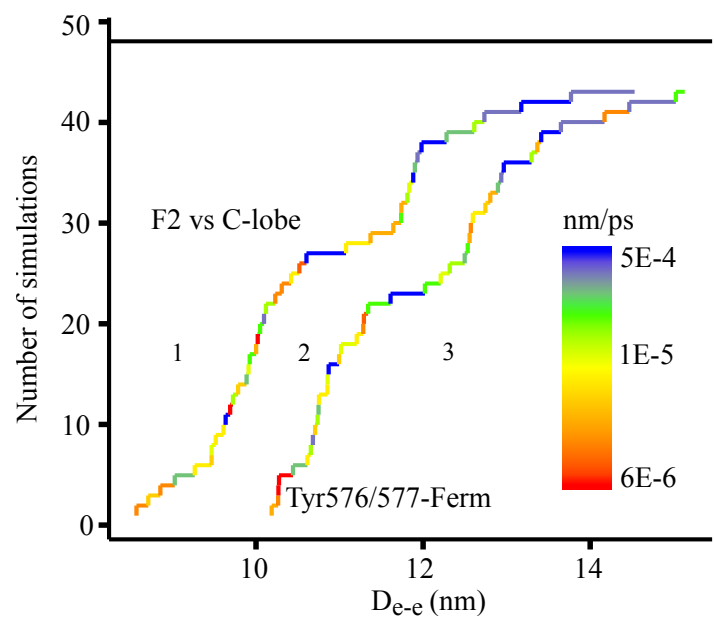

Figure 4.3 The accumulative number of simulations as a function of the distances between two pulling groups $\left(\mathrm{D}_{e-e}\right)$ when kinase C-lobe (left) and Tyr576-577 (right) are dissociated from FERM domain respectively. The color gradient is decided by the varying pulling velocities. main through the proline-rich linker onto the kinase C-terminus (Figure 4.1). Accordingly, in these simulations, a pulling force was applied to the basic patch of FERM and the C-terminus of the kinase domain in opposite directions with 12 different pulling velocities from $6 \mathrm{e}-6 \mathrm{~nm} / \mathrm{ps}$ to $5 \mathrm{e}-4 \mathrm{~nm} / \mathrm{ps}$. The slowest pulling simulation covered $1 \mu \mathrm{s}$. We observed the dissociation between the FERM and kinase domain in 43 out of 48 FPMD simulations and conformational damage of either the FERM F2-lobe or Kinase C-lobe in the remaining 5 simulations. The dissociation started from the region of the interface proximal to the points of force application, and resulted in the exposure of the critical activation loop (Figure 4.5). Exposure of the activation loop hosting the critical Tyr576/577 site (state 3 in Figure 4.5) indeed always required the dissociation of the F2-C lobes (state 2 in Figure 4.5), as we observed the release of Tyr576/577 from FERM always after, i.e. at larger distances $\left(\mathrm{D}_{e-e}\right)$ between two force application points, than the dissociation of the C-lobe from the F2-lobe (Figure 4.3).

As evident from Figure 4.5, we observed partial unfolding at the kinase C-terminus prior to exposure of Tyr576/577. The extent of unfolding prior to dissociation, however, is minor, 
comprising a maximally $15 \mathrm{~nm}$ increase in $\mathrm{D}_{e-e}$ (Figure 4.5 ) or 30 residues at C-terminus of the kinase domain. They belong to the first C-terminal $\alpha$-helix and an adjacent loop of the kinase domain (highlighted in red in Figure 4.4). This is remarkable, given that protein unfolding of the supposedly only moderately stable $\alpha$-helical F2 and Clobe structures competes with the dissociation from one another. According to the color gradient in Figure 4.3, which corresponds to varying loading rates in the range of two orders of magnitude, the significant unfolding before rupture was mostly ob-

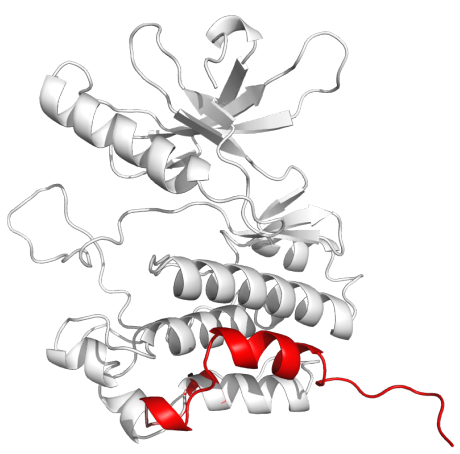

Figure 4.4 The unfolded helix induced by external force was highlighted in red in the structure of Kinase domain shown in cartoon. served in the simulations with high loading rates. Since the loading rates used in the simulations are about four to six orders of magnitude higher than the experimental loading rates, strong FERM or kinase distortion or unfolding, which are favored over dissociation in 5 of our 48 FPMD simulations, were suggested as artifacts from fast pulling. We thus conclude that force induces removal of the inhibitory FERM domain and kinase activation rather than unfolding of the domains, which would lead to kinase inactivation.

As we mentioned in Section 1.1, besides PIP $_{2}$, more binding partners of the FERM domain were suggested as stimuli for the FAK catalytic turnover, such as integrin [37] and growth factor receptors [38-40]. Although the docking sites in the FERM domain for these interactions are still unknown to date, we tested respective force applications on other potential docking sites in the FERM F1- and F3-lobes. As shown by our FPMD simulations with either two termini (Figure 4.6a) or the C-terminus and COM of F3-lobe (Figure 4.6b) serving as force application points, applying a stretching force to these sites leads to pronounced unfolding at the $\mathrm{C}$-terminus of kinase domain ( $>60$ amino acids) without occurrence of dissociation between the FERM and kinase domain. In contrast, only when applying force on the basic batch, unfolding does not exceed 30 amino acids before domain opening (Figure 4.4). Thus, the kinase domain can also feature an only mediocre stability against mechanical unfolding, i.e. the preference between FAK deactivation through kinase unfolding and its activation through FERM-kinase dissociation is strongly dependent on the points of force application. 


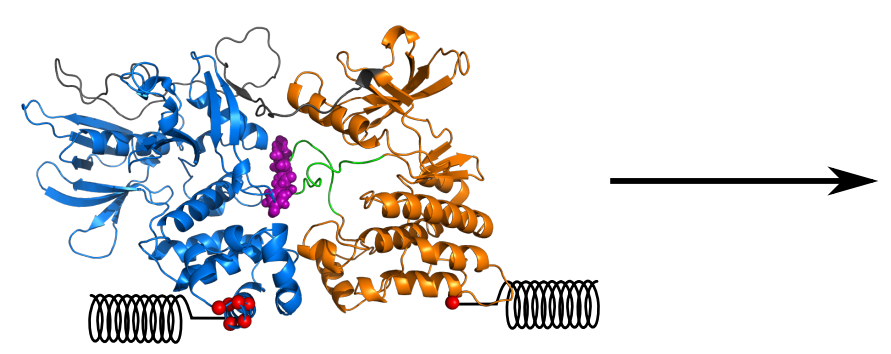

State 1

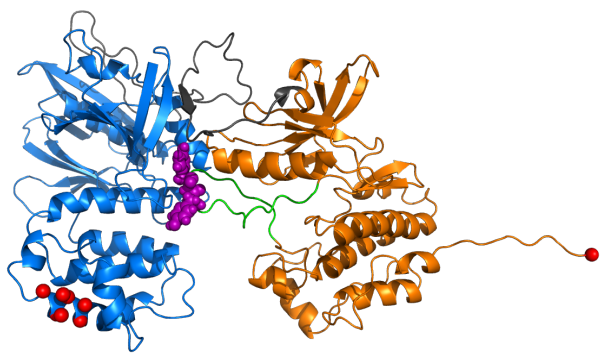

State 2

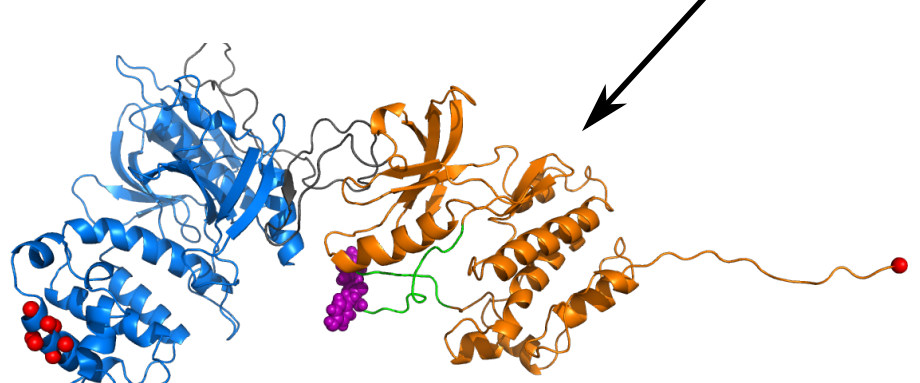

State 3

Figure 4.5 Mechanism of FAK activation by releasing the phosphorylation site, Tyr576/577, from the FERM domain under external force. Two virtual springs were applied at the basic (red) patch in the FERM and the C-terminal residue of the kinase domain in the initial protein structure. The C-lobe dissociated from FERM first, followed by the phosphorylation site, Tyr576/577. After only partial unfolding at the C-terminal site of the kinase domain, Tyr576/577 (violet) is released from the autoinhibition of FERM domain.

a)

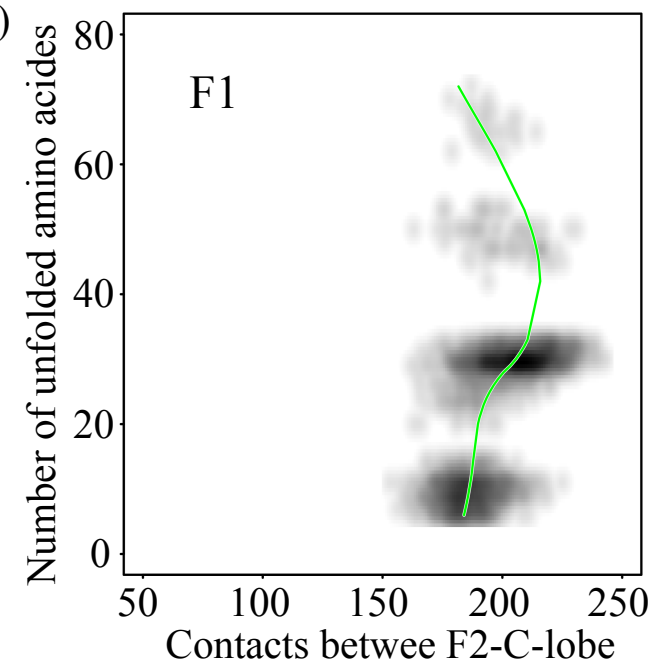

b)

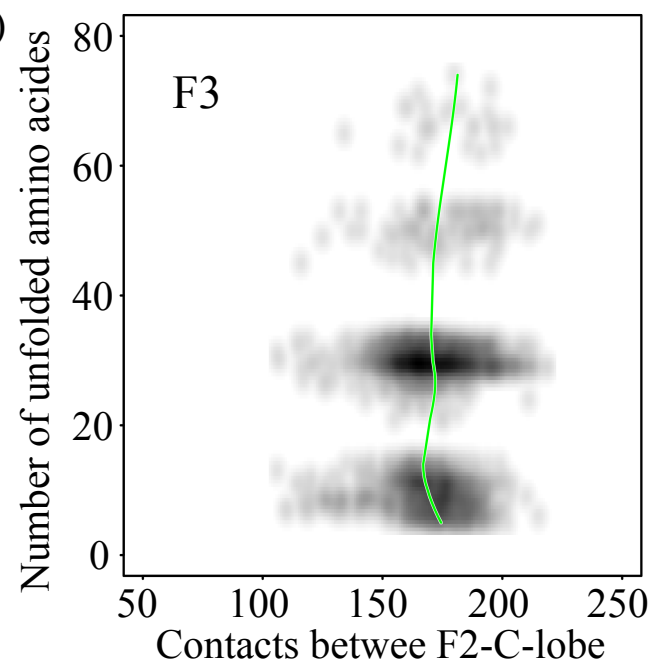

Figure 4.6 Unfolding dissociation for different force applications. The number of unfolded amino acids at the C-terminus of the FAK kinase domain is shown as a function of the number of contacts between the FERM F2- and kinase C-lobe (x-axis) in simulations with the external force applied to the N-terminus of the FERM F1-lobe (a) or the COM of the FERM F3-lobe (b). 


\subsection{PIP2 aggregation in POPE membrane bilayer}

At FAs, FAK is anchored to membranes through a specific interaction of the FERM basic patch with $\mathrm{PIP}_{2}$. We previously obtained a model for $\mathrm{PIP}_{2}$ bound FK-FAK and observed an allosteric change at the FERMkinase interface, but no full opening [41]. The question arises, if the force-induced full domain opening of FAK observed in solution also occurs when FAK is anchored to a membrane via $\mathrm{PIP}_{2}$. This would require both the $\mathrm{PIP}_{2}$-containing membrane as well as the $\mathrm{PIP}_{2}$-FERM link to be mechanically more robust against rupture than the FERMkinase interaction. To test this, we set up POPE membranes containing $1 \%, 10 \%$ and $15 \%(\mathrm{~mol} / \mathrm{mol})$ of $\mathrm{PIP}_{2}$ in the upper leaflet of the membrane, which was surrounded by water and neutralized by $\mathrm{CaCl}_{2}$.

To characterize the membrane structure in our simulations, the lipid surface area (A/lipid), which is a straightforward measure of the density of the membrane bilayer, was calculated for each system by dividing the overall membrane area by the number of lipids in the upper-leaflet of the membrane. The resulting areas per lipid from different simulations are listed in Table 4.1. The area/lipid from MD simulations of the membrane system with $1 \% \operatorname{PIP}_{2}(57.4 \AA /$ lipid $)$ is slightly smaller than the suggested reference value of the area/lipid of the POPE membrane in absence of $\mathrm{PIP}_{2}(58 \AA$ A/lipid $)$. A possible reason is
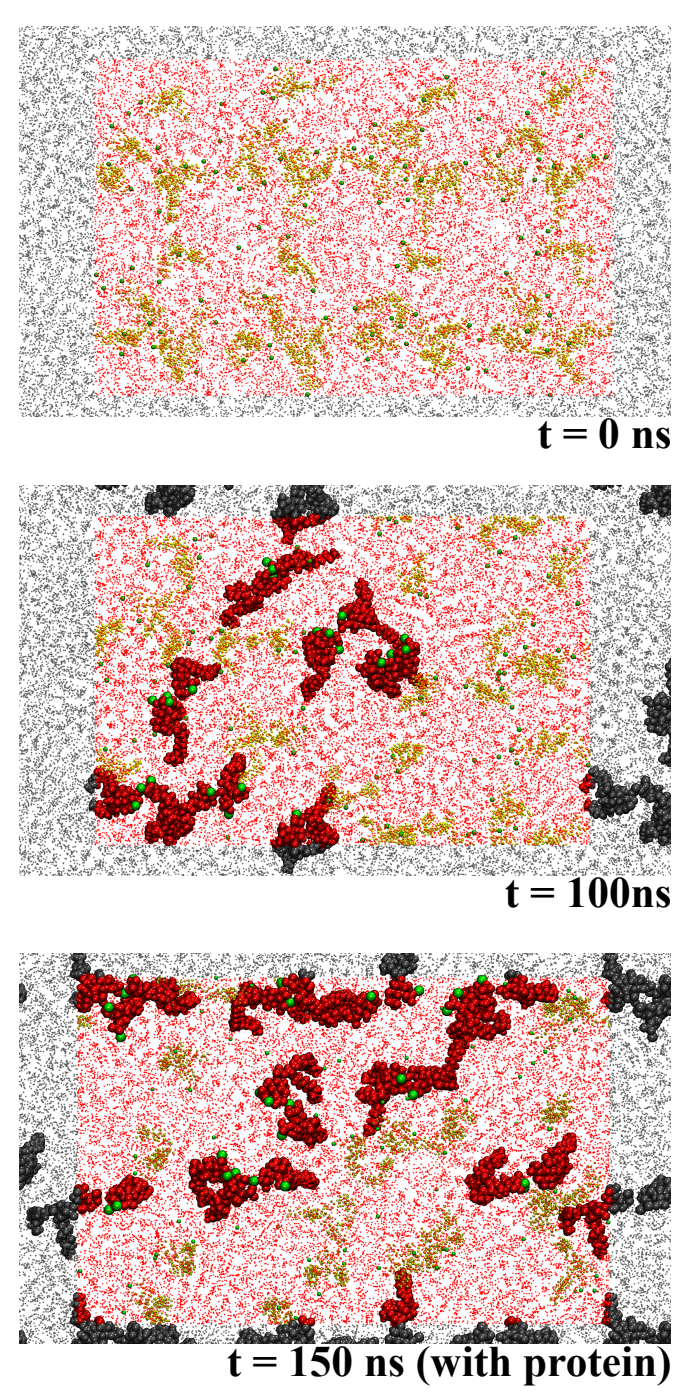

Figure 4.7 Clustering of $\mathrm{PIP}_{2}$ as observed during MD simulations of the membrane containing $15 \% \mathrm{PIP}_{2}$ lipids. $\mathrm{PIP}_{2}$ clusters (spheres) formed from indicidual $\mathrm{PIP}_{2}$ lipids (yellow spheres) and are neutralized by $\mathrm{Ca}^{2+}$ ion (green sphere, top). After $100 \mathrm{~ns}$ of MD simulations, $35 \% \mathrm{PIP}_{2}$ lipids mediated by $\mathrm{Ca}^{2+}$ clustered (middle). After further $150 \mathrm{~ns}$ of MD simulations in the presence of protein, $50 \% \mathrm{PIP}_{2}$ lipids got clustered (bottom). 
an effect of $\mathrm{PIP}_{2}$ on the local area of the membrane surface [117]. Indeed, we observed a further decrease of area/lipid when increasing the $\mathrm{PIP}_{2}$ concentration (Table 4.1).

$\mathrm{PIP}_{2}$ is known to enrich at FAs resulting in high local $\mathrm{PIP}_{2}$ concentrations $[41,103]$. Previous studies showed $\mathrm{PIP}_{2}$ cluster formation to be mediated by divalent cations such as $\mathrm{Ca}^{2+}$ [107]. Thus, we traced the formation of PIP 2 clusters in POPE membranes upon different $\mathrm{PIP}_{2}$ concentrations and protein binding. All the MD simulations started from individual $\mathrm{PIP}_{2}$ molecules (Figure 4.7 top). Within $100 \mathrm{~ns}$ of MD simulations of the membrane containing $15 \% \mathrm{PIP}_{2}$ lipids, we observed the formation of small $\mathrm{PIP}_{2}$ clusters (see Section 4.2.2) involving two or more lipids and $\mathrm{Ca}^{2+}$ (Figure 4.7, middle), accompanied by a decrease of area per lipid by $\sim 1 \AA^{2}$. Clustering was less pronounced for smaller PIP 2 concentrations ( $1 \%$ and $10 \%$ ) (Table 4.1). Subsequently, the protein was anchored to membranes with $1 \%$ and $15 \% \mathrm{PIP}_{2}$. In both cases, and independent from the initial rotation of the protein relative to the membrane plane, the protein remained stably bound to the membrane through the FERM-PIP 2 link and additional favorable electrostatic interactions that spontaneously and reproducibly formed between the kinase C-lobe and the membrane (Figure 4.8). The protein-membrane interactions induced further increase of PIP $_{2}$ clustering and decrease of the area per lipid in the membrane (Table: 4.1). We observed the clusters of $\mathrm{PIP}_{2}$ to often have a string-like shape, which is consistent with a previous computational study [118].

Table 4.1 Structural properties of the membranes with different concentrations of $\mathrm{PIP}_{2}$ lipids.

\begin{tabular}{c|c|c|c} 
Systems & $*$ Area/lipid & $*$ Aggregated PIP $2 \%$ & $*$ Nr. of clusters \\
\hline POPE $+3 \mathrm{PIP}_{2}$ & $57.36 \pm 0.0060$ & 0 & 0 \\
POPE $+10 \% \mathrm{PIP}_{2}$ & $56.81 \pm 0.0056$ & $15 \%$ & 2 \\
POPE $+15 \% \mathrm{PIP}_{2}$ & $56.40 \pm 0.0061$ & $35 \%$ & 7 \\
POPE $+15 \% \mathrm{PIP}_{2}+$ Protein & $55.38 \pm 0.0100$ & $50 \%$ & 8 \\
\hline
\end{tabular}

*see Section 4.2.2

\subsection{FAK-membrane interactions}

To monitor the mechanical response of membrane-anchored FK-FAK, extensive FPMD simulations were carried out for the membrane-protein systems containing high (15\%) or low (1\%) $\mathrm{PIP}_{2}$ concentrations with varying directions of force application. External force was applied to the protein in form of a moving harmonic spring attached to the kinase $\mathrm{C}$-terminus 
with constant velocity along a direction vertical or diagonal to the membrane (Figure 4.2), while position restraining the center-of-mass of the membrane bilayer.

When an external force was applied to FK-FAK anchored to a membrane at $15 \% \mathrm{PIP}_{2}$ concentration, independent of the pulling direction, we observed a loss of contacts of the kinase domain with the membrane and with the FERM domain, while the FERM-membrane interaction remained intact (upper half of Figures 4.8 and 4.9). While diagonal pulling dissociates the kinase domain from the membrane and from the FERM domain concurrently, vertical pulling results in a kinase-membrane dissociation (inactive and bound FK-FAK, $i$ and $b$ ) prior to kinase-FERM dissociation (active FK-FAK, $a$ ). In none of these simulations, we observed kinase unfolding prior to dissociation. Also, for both pulling directions, the membrane and the $\mathrm{PIP}_{2}$-FERM interaction were mechanically more robust than the FERMkinase interface. Thus, the membrane simulations reproduced the process predominantly observed for FK-FAK in solution (compare Figures 4.3 and 4.5), namely the force-induced removal of the auto-inhibitory FERM domain and exposure of the activation loop carrying the Tyr576/577 phosphorylation site.

The anchorage of FK-FAK to a membrane containing only $1 \% \mathrm{PIP}_{2}$ occurs through a single $\mathrm{PIP}_{2}$ molecule. When force is applied to FK-FAK in diagonal direction of the membrane, only the loss of connects between the kinase domain and the membrane was observed under force (inactive and bound FK-FAK, $b$ ), accompanied by a horizontal translocation of FK-FAK along the membrane (lower half of Figure 4.8 and 4.9), that is to say, the external force only functioned on FK-FAK in horizontal direction. As shown in Figure 4.8, force application in vertical direction leads to the detachment of the kinase domain from the membrane, followed by the detachment of also the FERM domain (inactive and unbounded FK-FAK, $u$ ). Full loss of membrane anchoring naturally stops force transmission and impedes activation. Thus, an interaction of the FERM basic patch with a single $\mathrm{PIP}_{2}$, which is likely at low $\mathrm{PIP}_{2}$ concentrations, is insufficient for mechanical FK-FAK activation. Only a membrane containing clusters of multiple $\mathrm{PIP}_{2}$ lipids can provide a sufficiently stable membrane-anchorage of the FERM domain outlasting the exposure of the activation loop of FK-FAK under force. The pulling direction at these high $\mathrm{PIP}_{2}$ concentrations, instead, appears to be less relevant. 
diagonal (multiple $\mathrm{PIP}_{2}$ ):
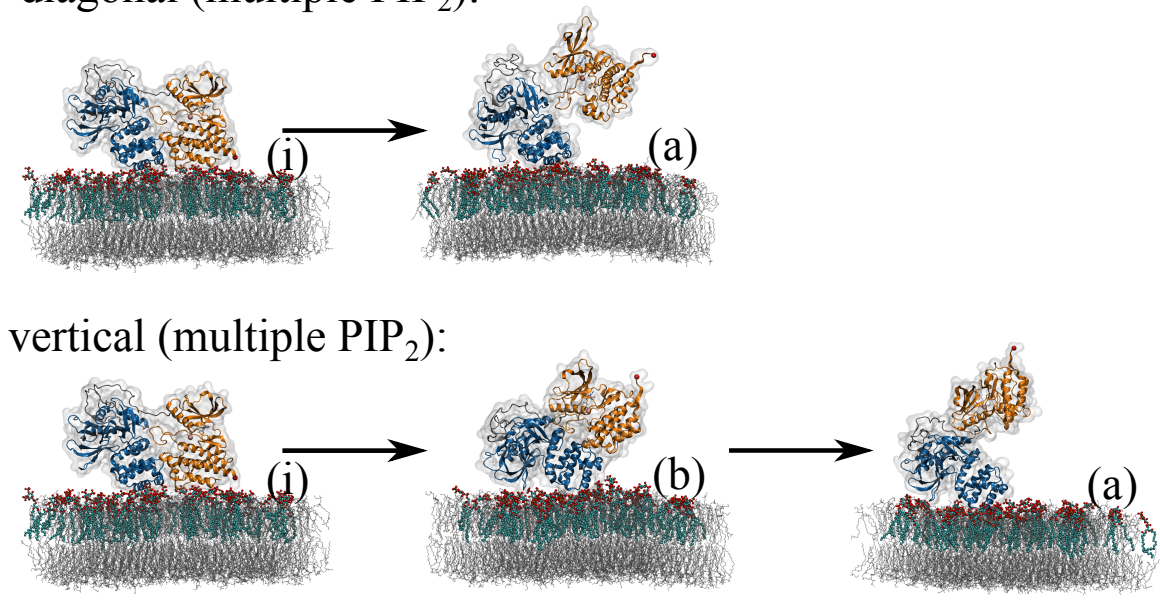

diagonal (single $\mathrm{PIP}_{2}$ ):
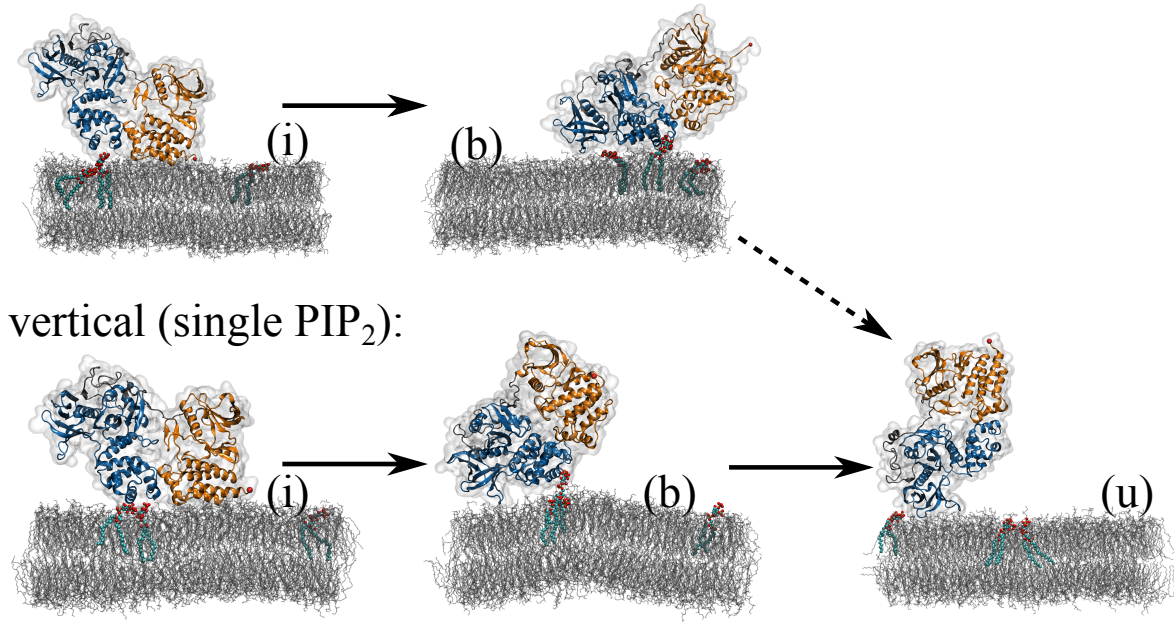

Figure 4.8 Mechanism of FAK activation in presence of the membrane bilayer by releasing the phosphorylation site, Tyr576/577, from the FERM domain (blue cartoon) under external force. One virtual spring attached to the COM of the membrane bilayer (gray) kept the membrane in its initial position, whereas the other spring at the $\mathrm{C}$-terminal residue pulled the kinase domain (orange cartoon) in vertical $\left(90^{\circ}\right)$ or diagonal $\left(45^{\circ}\right)$ direction away from the membrane with multiple $(15 \%)$ or signal $(1 \%)$ PIP $_{2}$. The labels, i, a, b and u, correspond to the inactive states of FK-FAK as shown in Figure 4.9. 


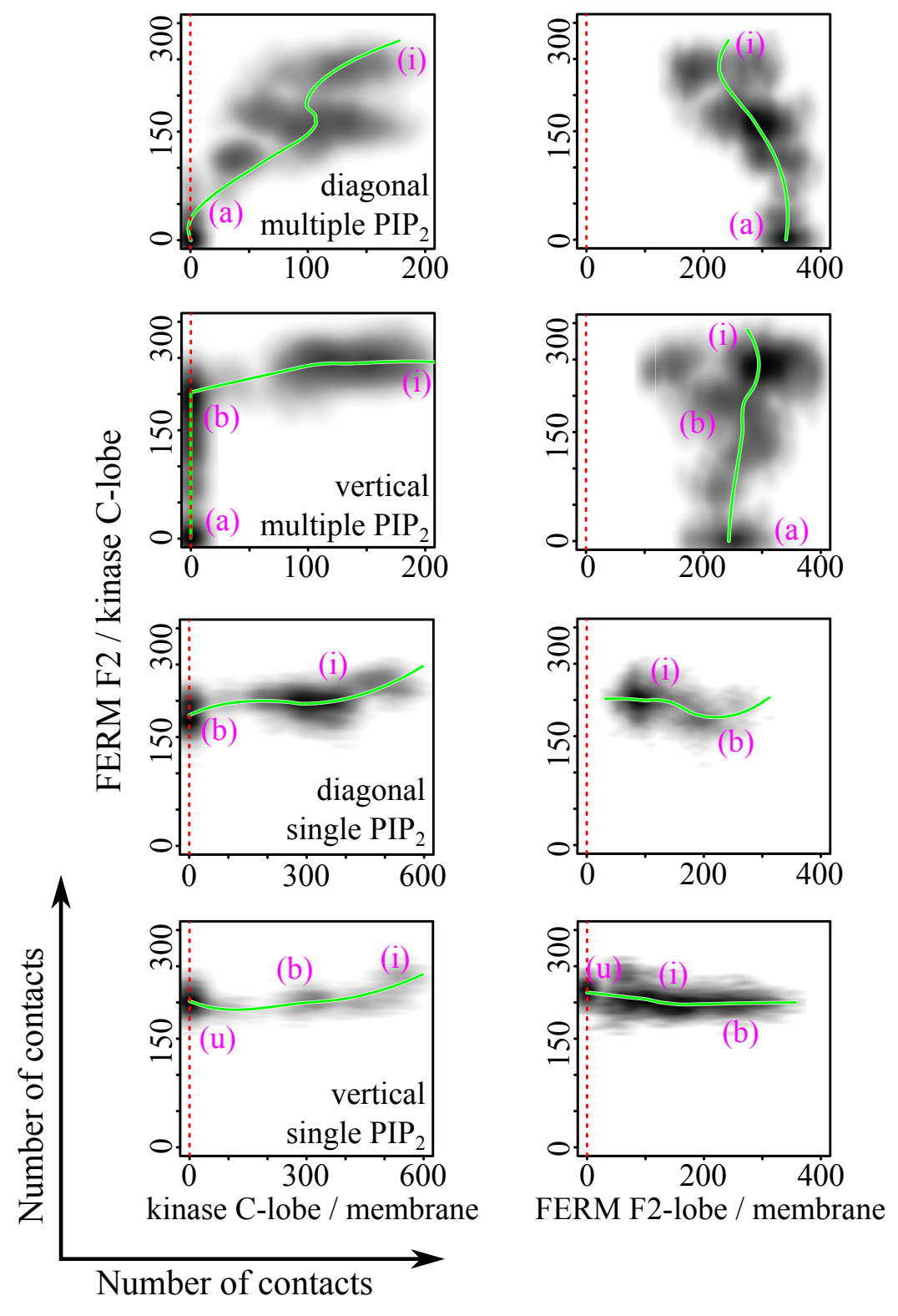

Figure 4.9 Dissociation mechanisms of FK-FAK under force at varying pulling directions and $\mathrm{PIP}_{2}$ concentrations. The number of contacts between the FERM F2- and the kinase C-lobe is shown as a function of the number of contacts between kinase domain and membrane or between FERM domain and the membrane for all simulations of the FAK-membrane system with a pulling velocity of 0.03 $\mathrm{nm} / \mathrm{ns}$. The labels, $\mathrm{i}, \mathrm{a}, \mathrm{b}$ and $\mathrm{u}$, in purple correspond to the inactive, active, bound and unbound states of FK-FAK as shown in Figure 4.8. The red dash lines illustrate the behavior of FK-FAK in free form. The green solid lines are the polynomial fits. 


\subsection{Mechanism of force-induced FK-FAK opening}

\subsubsection{Collective opening motion of FK-FAK}

We next analyzed in further detail the dynamics underlying the force-triggered FK-FAK domain-domain rupture. Partial least square functional mode analysis (PLS-FMA) was carried out to test if the dissociation between the FERM F2 and the kinase C-lobe follows the same mechanism in absence and presence of the membrane. This method is described in section 2.5. In this work, the time-dependent functional variable $Y$ was the separation between F2- and C-lobe, reflecting the closing-to-opening motion of FK-FAK (black line in Figure 4.10b). $X(t)$ were the collective coordinates of all backbone atoms of FK-FAK along the concatenated simulation trajectories.

From the concatenated trajectories of FPMD simulations in absence of the membrane, the first 50000 frames (marked in red in Figure 4.10b) were used to compute the PLS vector $b$, by considering all possible pulling velocities (model building part). The remaining 500000 frames of FPMD simulation trajectories in absence of membrane (green in Figure $4.10 b$, model validation part I) and about 8000 snapshots of FPMD simulations in presence of membrane (blue in Figure 4.10b, model validation part II) were used to validate the computed $b$.

The PLS vector $b$ was a combination of uncorrelated PLS components. By increasing the number of PLS components, the correlation of dissociation processes in different simulations, including model building part (red), validation part I (green) and validation part II (blue), was monitored and shown in Figure 4.10a. When considering about 11 PLS components, we obtained a collective motion of FK-FAK in solution form (cyan) to opening form (grey) that maximally correlates with the increase in minimal distance between the F2 and C-lobes (Figure 4.10c). A very strong correlation larger than 0.9 was also obtained for the trajectories of membrane-bound FAK-FK, suggesting that the PLS components capture the essential opening dynamics of FK-FAK both in solution and when membrane-bound. This agreement of the observed dynamics also implies that the simplified system of FK-FAK in solution shows a FERM-kinase dissociation mechanism highly similar to the one in the more realistic membrane system, even though it lacks effects from FERM/kinase-membrane interactions. 


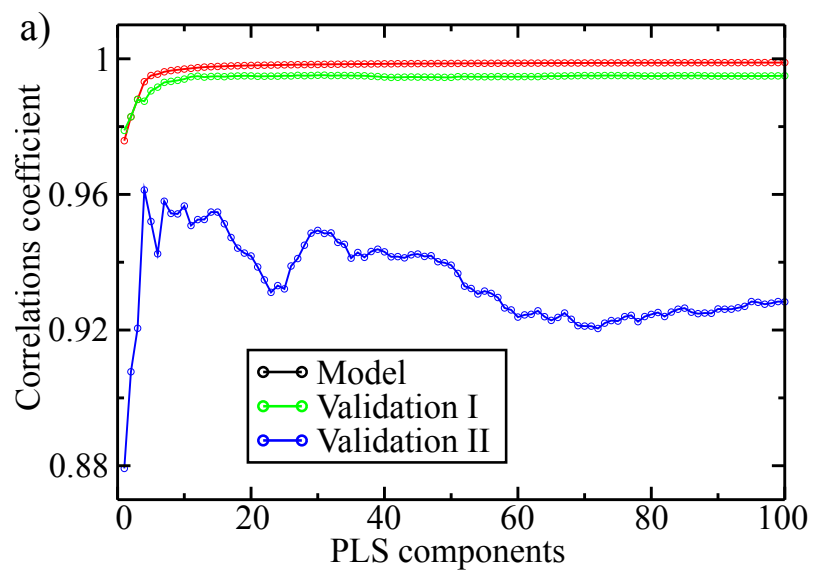

c)
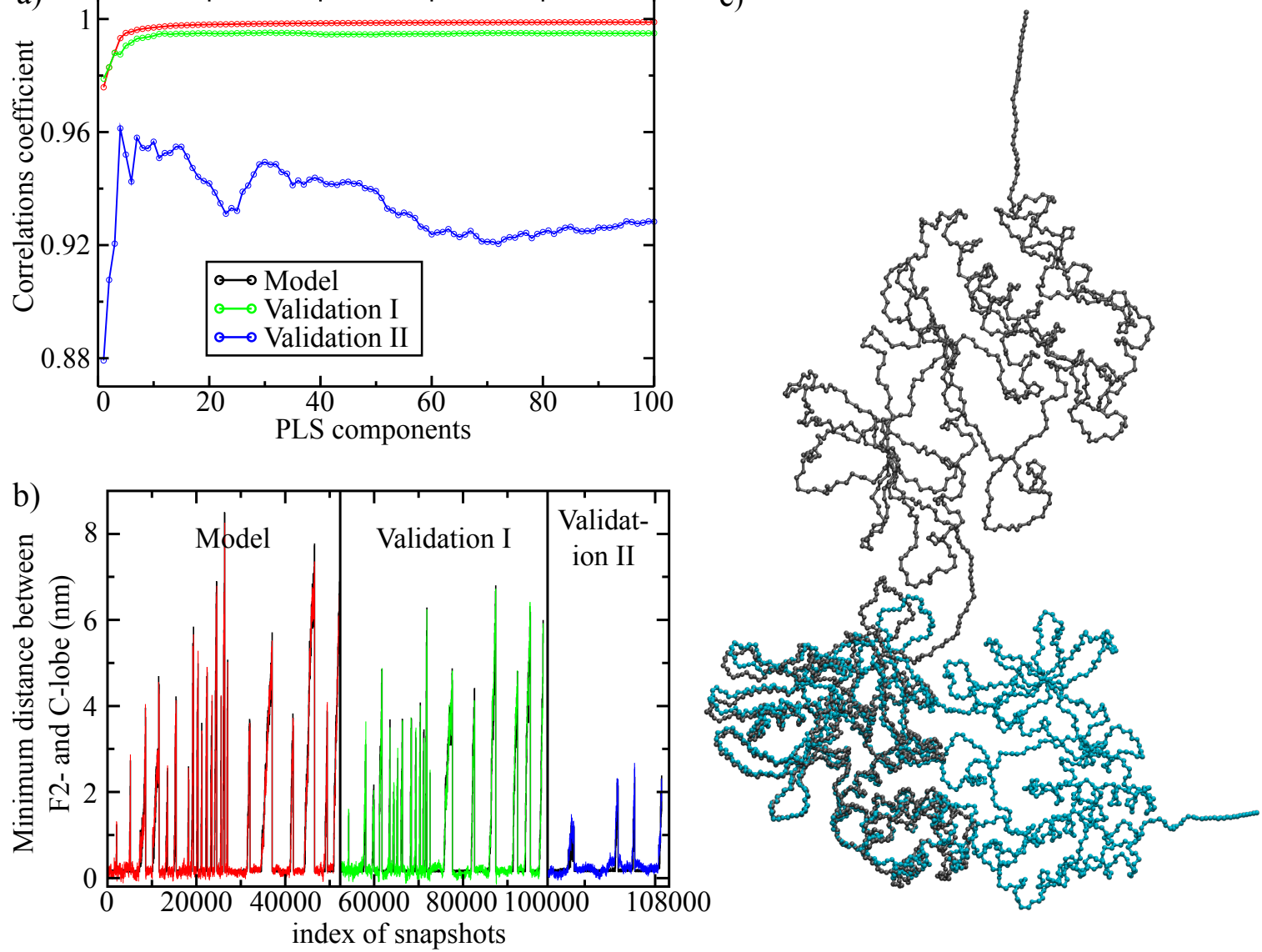

Figure 4.10 Partial least square functional mode analysis. (a) Correlation of dissociation processes in the simulations in the presence or the absence of the membrane bilayer. (b) Projection of minimum distances between F2- and C-lobe with 11 PLS components for all concatenated trajectories on the minimum distances obtained from FPMD simulations. (c) Collective motion of FK-FAK from a closed form (cyan) to an open form (grey) by considering 11 PLS components. 


\subsubsection{Identifying force-carrying interactions at FERM F2- and kinase C- lobe}

We next analyzed in further detail in the opening mechanism of the F2/C-lobe interface to identifying the interactions giving primarily rise to the rupture force. Figure 4.11 shows the force profiles and F2/C-lobe interaction areas as a function of spring dislocation for a subset of trajectories. Independent from the loading rate and for both FK-FAK in solution and bound to the membrane, we observed a reduction in interaction area between the two lobes (black) in two steps, both of which coincide with noticeable force peaks (blue). The

a) no membrane
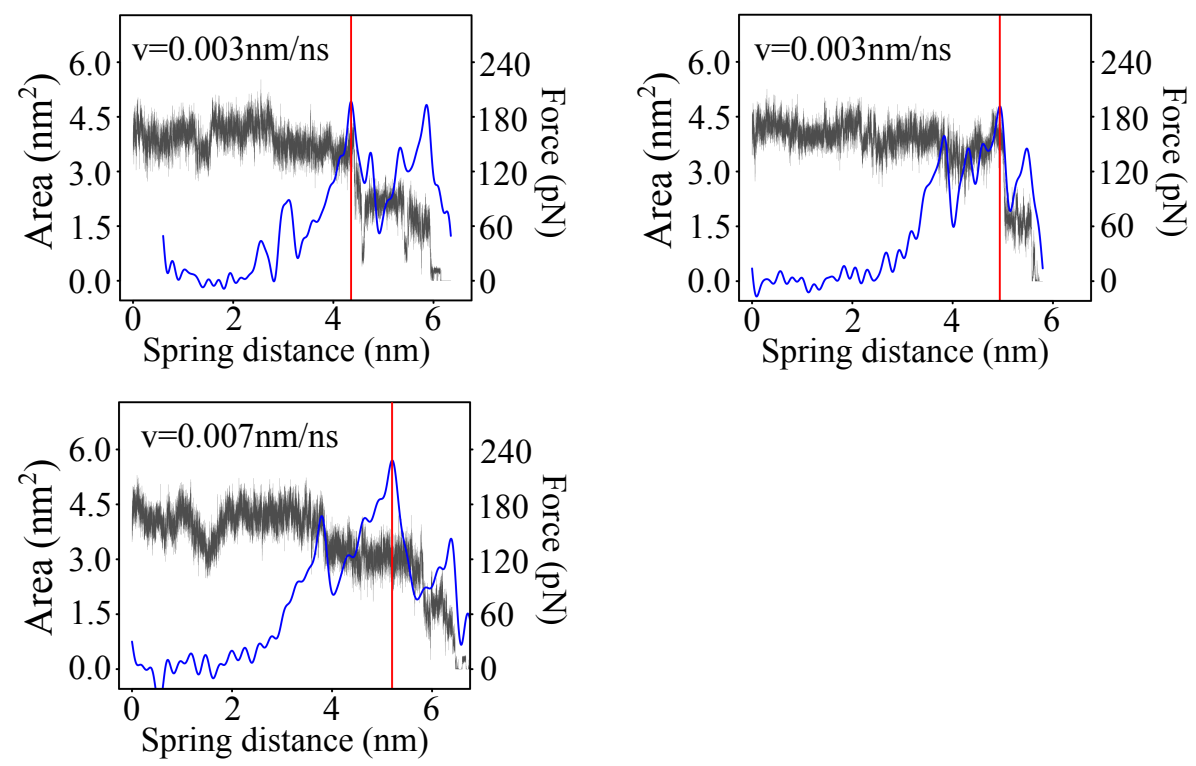

b) with membrane
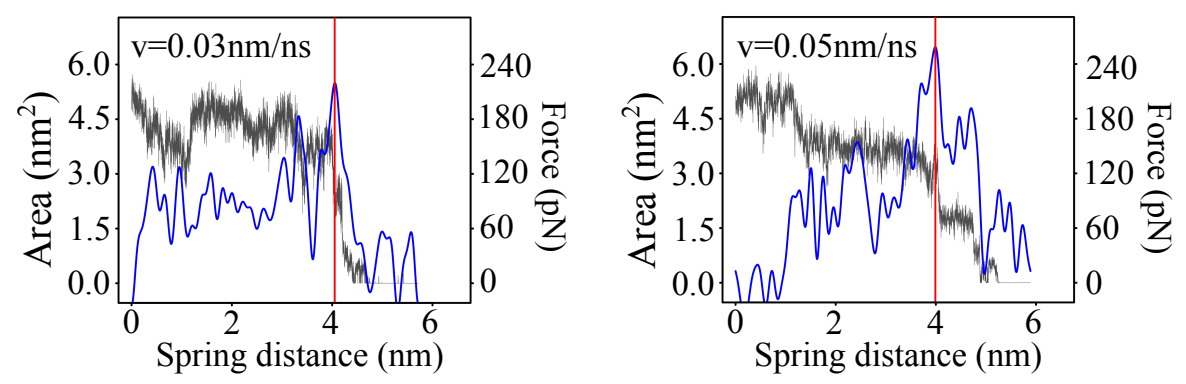

Figure 4.11 Interfacial area between the F2- and C-lobe and overlayed force profile as a function of spring distance. The red lines indicate the moment when the force reaches a maximum coinciding with a first drop in interfacial area from first (3-4.5 $\left.\mathrm{nm}^{2}\right)$ to second step (1.5-2.8 $\left.\mathrm{nm}^{2}\right)$. 
maximal force is reached when the first decrease (from 3-4.5 $\AA$ to 1.5-2.8 $\AA$ ) in inter-lobe area occurs (red solid line). This leads to a short-lived intermediate, as reflected by a second peak in the distribution of the F2/C-lobe interface area (Figure 4.12), until full dissociation of the two lobes occurs. We note that the intermediate becomes less evident for faster pulling velocities.

According to the auto-inhibitory conformation of FK-FAK [36], the interaction between the FERM F2- and kinase C-lobe comprises two parts, a hydrophobic pocket (Y180, M183, V196, L197 and F596) and a set of hydrogen bonds and saltbridges. In the FPMD simulations, some of the residues at the $\mathrm{F} 2 / \mathrm{C}$-lobe interface were even observed to swap their interacting partners, complicating the analysis of the mechanism of the dissociation process. However, identifying the contribution of each residue at the F2/C-lobe interface to the resistance to the domain opening

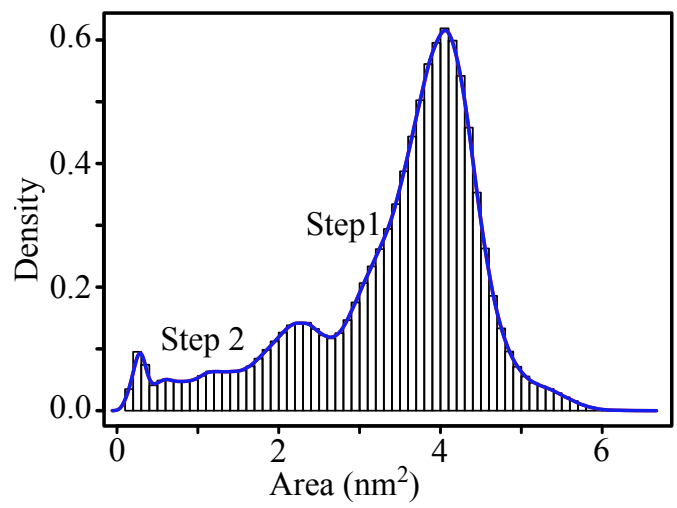
is helpful for understanding the mechanical activation of FAK. Therefore, TRFDA was carried out (see Section 2.3).

By means of TRFDA, the vector pairwise force representing the non-covalent interactions between residues $r i$ in the FERM F2-lobe and $r j$ in the kinase C-lobe at each simulation step was calculated by according to Equation 2.13. Summing up the absolute values of the vector pairwise forces acting on $r i$ or $r j$ in the direction of force application (Equation 2.15) gave the punctual stress (see section 2.3) at the F2/C-lobe interface induced by the external force. The punctual stress represents here the non-bonded interactions of residue $r i$ in kinase domain with the FERM domain or vice versa. According to the change in punctual stress of the residues in the FERM or kinase domain over the simulation time, the role of each interface residue during the dissociation steps can be monitored. Note that a quantitative comparison between the internal punctual stress and the externally probed rupture force is not straightforward, although they are expressed in the same units. However, the decay of punctual stress coincides with the increase of rupture force (compare Figure 4.13 and 4.11). The red line in Figure 4.13, which indicates the maximal force in the relevant 
force profile, separates the punctual stress profile into two parts. Remarkably, these two parts are consistent with the two steps of decrease in the interfacial area induced by the external force (Figure 4.12). Before reading the rupture force (step 1), residues largely maintain their interaction with the other domain, whereas some of them lost the interactions right when the rupture force has been reached (right of the red line), leading to an intermediate with only some F2/C-lobe interactions still maintained. Thus, we can conclude that the residues, which lose their interactions with the other domain at step 1, contribute most to the occurrence of the maximal force. These residues are crucial for the FAK mechanical stability.

TRFDA was carried out for three non-membrane FPMD in low pulling velocities and two complex FPMD simulations in diagonal pulling direction. The probability of a residue at domain interface to lose its interaction with the other domain in the two dissociation steps was counted and is listed in the Table 4.2. If a residue was identified in more than 4 simulations at the same step, it is colored in green, blue and cyan (for step 1) or in magenta (for step 2).

Based on the results in Table 4.2, inter-lobe interactions reproducibly rupturing at one of the two dissociation steps are highlighted in Figure 4.14a. The first major rupture step requires the breakup of a hydrophobic cluster composed of residues Y180, M183, N193, V196, and F596 and of an additional salt bridge (D200-R598, Figure 4.14b). All of these interactions remained intact during the five simulations not showing dissociation but unfolding of FK-FAK, while in the other cases (90\% of trajectories), their rupture gave rise to the maximal force, stressing the critical stabilizing role of this hydrophobic cluster. In agreement, mutations Y180A, M183A and F596D resulted in a constitutively active FAK due to the opening of the FERM-kinase interface [36]. Residue pairs rupturing at the second step are located further away from the points of force application, i.e. the membrane anchor, and include residues of mostly electrostatic nature (E182, R184, K190 and N595, N628, N629, E636). This second rupture step is immediately followed by the opening of the remaining FERM-kinase interface established between the FERM F1 and the kinase N-lobe, including the exposure of Tyr576/577. Thus, the rupture process resembles a zipper-like mechanism, during which the FERM and kinase interface is sequentially opened. Herein, the membraneproximal hydrophobic patch around F596 represents the most robust mechanical clamp to be opened first. 
A) without membrane $(\mathrm{v}=0.003 \mathrm{~nm} / \mathrm{ns})$

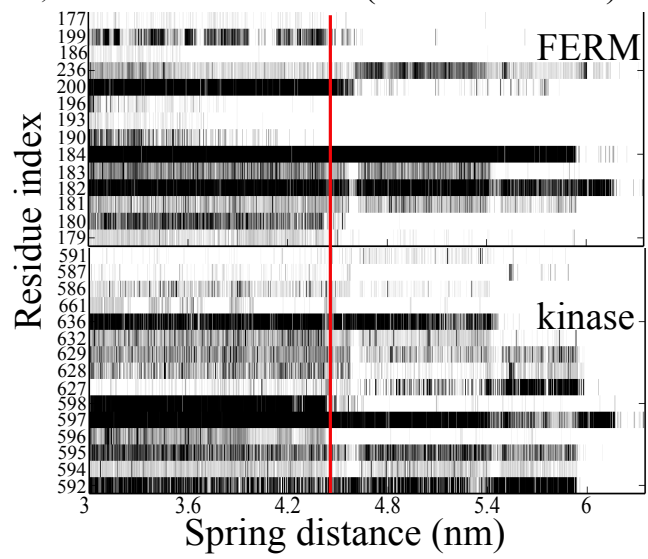

without membrane $(\mathrm{v}=0.007 \mathrm{~nm} / \mathrm{ns})$

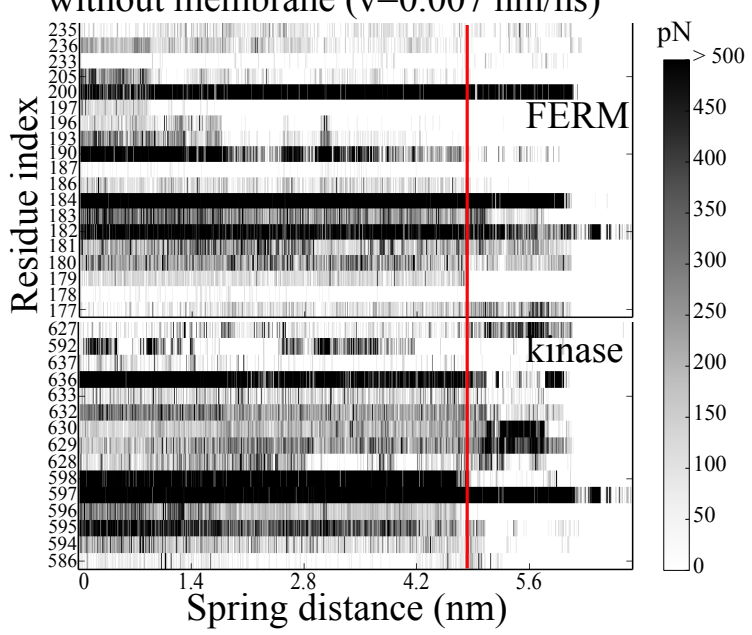

B) with membrane $(\mathrm{v}=0.03 \mathrm{~nm} / \mathrm{ns})$

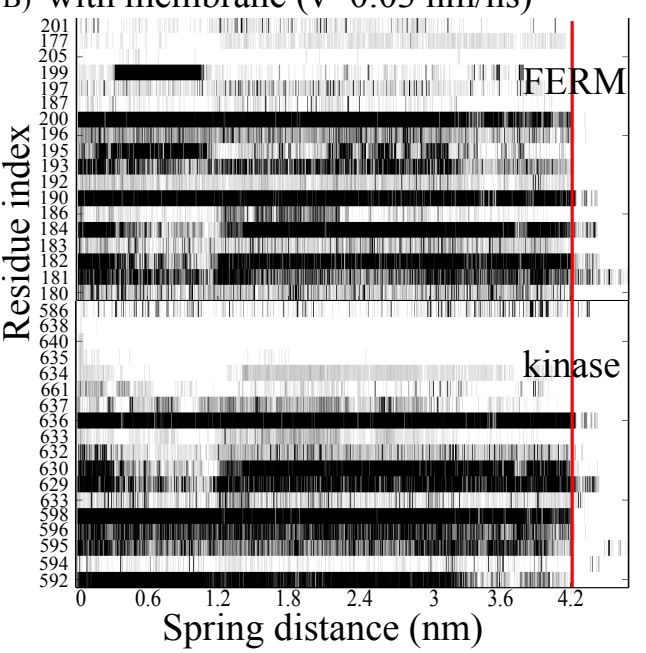

without membrane $(\mathrm{v}=0.003 \mathrm{~nm} / \mathrm{ns})$

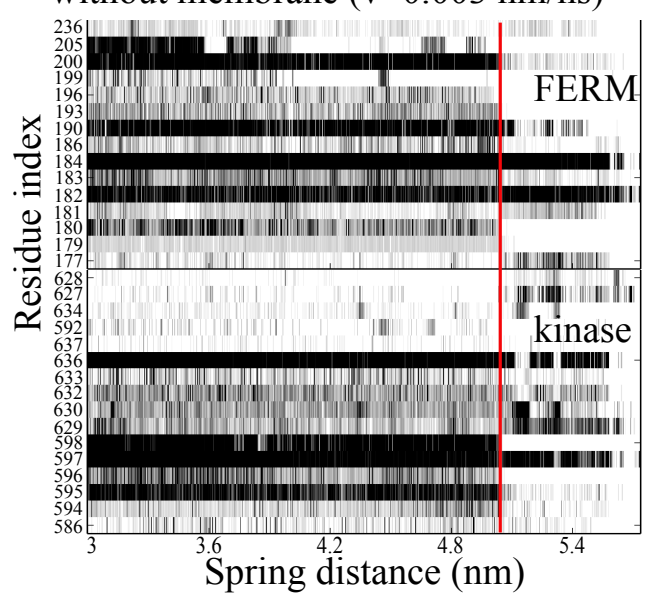

Spring distance $(\mathrm{nm})$ with membrane $(\mathrm{v}=0.05 \mathrm{~nm} / \mathrm{ns})$

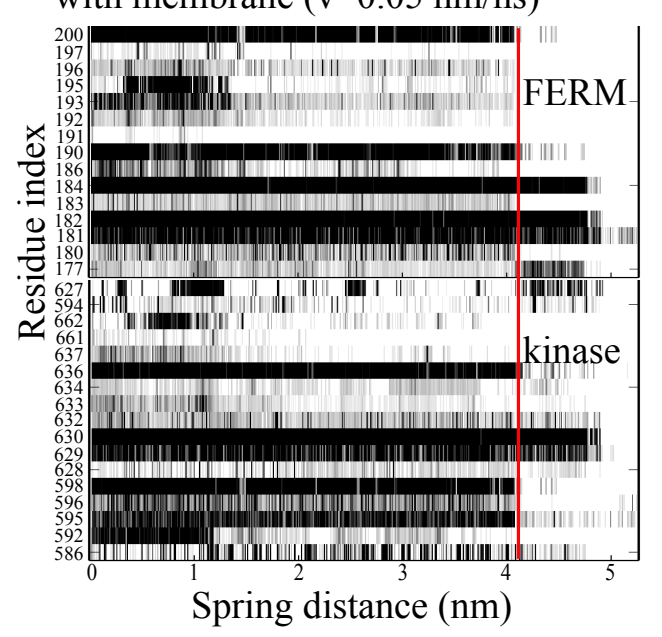

Figure 4.13 The color gradient represents the punctual stress $(>50 \mathrm{pN})$ acting on the residues at F2/C-lobe interface as a function of spring distance in unit of force. The red line indicated the maximal force in the relevant force profile for three non-membrane FPMD in low pulling velocities and two complex FPMD simulations in diagonal folling direction. 
Table 4.2 The probability of a residue in the FERM (left) and kinase (right) domains to lose its interaction with the other domain in one of the two major dissociation steps, step 1 and step 2, based on the TRFDA results (Figure 4.13). Residues dominating the first rupture step are shown in green (hydrophobic interaction), blue (salt bridges) and cyan (varying partners), and residues dominantly involved in the second step in magenta (mostly electrostatic interactions). Compare Figure 4.14.

\begin{tabular}{c|c|c|c|c|c|c|c}
\hline FERM & step 1 & step 2 & elsewhere & kinase & step 1 & step 2 & elsewhere \\
\hline R177 & 0.2 & 0.6 & 0.2 & I586 & 0.2 & 0.8 & \\
S179 & 0.6 & & 0.4 & E592 & 0.2 & 0.2 & 0.6 \\
Y180 & 0.8 & 0.2 & & I594 & 0.2 & 0.6 & 0.2 \\
W181 & 0.2 & 0.8 & & N595 & 0.2 & 0.8 & \\
E182 & & 1 & & F596 & 1 & & \\
M183 & 1 & & & R597 & & 0.6 & 0.4 \\
R184 & & 1 & & R598 & 1 & & \\
N186 & 0.8 & & 0.2 & K627 & & 0.6 & 0.4 \\
K190 & 0.2 & 0.8 & & N628 & & 0.8 & 0.2 \\
S192 & 0.4 & & 0.6 & N629 & & 1 & \\
N193 & 0.8 & & 0.2 & D630 & 0.2 & 0.6 & 0.2 \\
E195 & 0.4 & & 0.6 & I632 & 0.2 & 0.8 & \\
V196 & 1 & & & G633 & 0.2 & 0.6 & 0.2 \\
L197 & 0.2 & & 0.8 & R634 & 0.2 & 0.4 & 0.4 \\
K199 & 0.6 & & 0.4 & E636 & & 1 & \\
D200 & 0.8 & 0.2 & & N637 & 0.6 & & 0.4 \\
R205 & 0.4 & & 0.6 & Y661 & 0.2 & 0.2 & 0.6 \\
N235 & & 0.2 & 0.8 & D662 & 0.2 & & 0.8 \\
R236 & & 0.6 & 0.4 & & & & \\
\hline
\end{tabular}



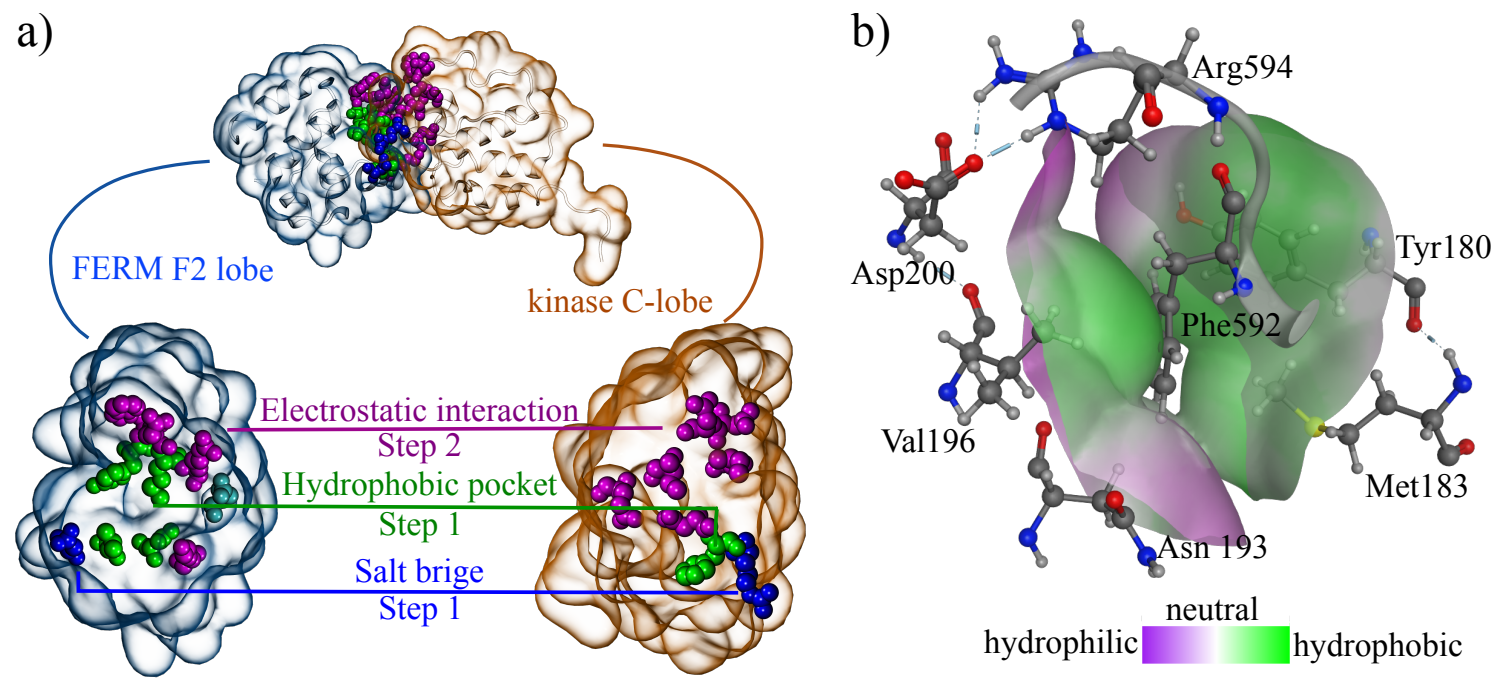

Figure 4.14 Based on the TRFDA results, the residues at the domain interface were classified according to the moment when they lose their interactions with the other domain. (a) Residues dominating the first rupture step (Figure 4.12 and 4.11) are shown in green (hydrophobic interaction), blue (salt bridges) and grey (varying partners), and residues dominantly involved in the second step (Figure 4.12 and 4.11) in magenta (mostly electrostatic interactions). (b) The major force-carrying hydrophobic pocket including Y180, M183, N193, V196, and F596 and the salt bridge (D200-R598). 


\subsection{Force-dependent activation rates}

According to our MD simulation data, the auto-inhibition of FAK can be relieved by mechanical force. The question arises if the increase in activation rate expected from our simulations is in line with the experimental observations of FAK activation in cells under stretching conditions. We obtained maximal rupture forces, i.e. forces for FAK FERMkinase dissociation (compare Figure 4.11) from the 43 trajectories showing dissociation, at twelve different loading rates covering two orders of magnitude (Figure $4.15 a$, black dots). FK-FAK activation forces varied at our MD time scales between 190 and $350 \mathrm{pN}$, which covers a range below the one observed for titin kinase $(400 \mathrm{pN}$ at $0.4 \mathrm{~m} / \mathrm{s})$, a kinase known to be mechanically activated by forces present in muscle [47, 109, 119]. Our calculated fore regime is similar or slightly lower than those predicted by MD simulations for the focal adhesion proteins talin and vinculin (250-400 $\mathrm{pN}$ for nanosecond scale activation of talin [6] and $100 \mathrm{pN}$ for sub-nanosecond activation of vinculin [11]), respectively.
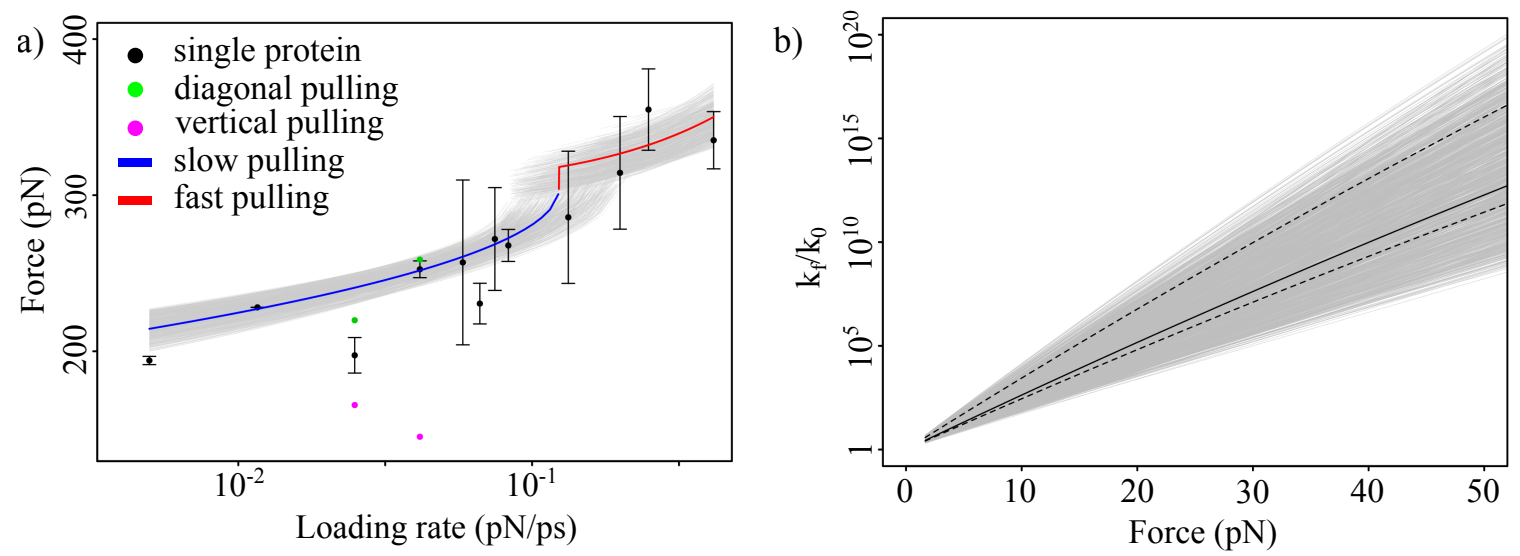

Figure 4.15 DHS fitting of rupture forces. (a) Rupture forces obtained by FPMD simulations of FAK only (see Section 2.1.3 black) as a function of the logarithm of the loading rate $\mathrm{L}$. The gray area represents the possible prediction provided by DHS model (see Section 4.2.3). For the best fit, we obtained a residual root-mean square deviation (RMSD) of $17 \mathrm{pN}$, and the gray area represents fits with up to $20 \mathrm{pN}$ of RMSD. Rupture forces determined from the FPMD simulations of membranebound FAK are shown in green (for diagonal pulling) and magenta (for vertical pulling). The DHS model gives different loading rate dependences at the slow (blue) and fast pulling regimes, which are separated by the critical loading rate. (b) The force-dependent activation rate of FAK, $k_{f}$, as obtained from the DHS model with parameters from the fit in (a), normalized by its activation rate at zero force, $k_{0}$. As in (a), the gray area shows the predictions within an RMSD of $<20 \mathrm{pN}$. The dashed lines indicate the upper and lower limits of the fits obtained from the estimated error ranges in Table 4.3. 
The Dudko-Hummer-Szabo (DHS) model was employed to estimate force-dependent FAK dissociation rates $k_{f}$ at the more relevant lower force regime (see Sections 1.3 and 4.2.3). This model takes the shape of the free-energy barrier and its shift under forces into account by introducing an additional parameter $v$, with $\langle F\rangle \sim(\ln V)^{v}$. RMSD values between predicted forces and the obtained rupture forces from MD simulations obtained for 500000 Monte Carlo random steps was used to score the fitting with the DHS model. It is shown as a function of $L_{c r i t}$ in Figure 4.16. The best fit yielding the smallest $R M S D$ provided the estimations of a distance to the transition state, $X_{b}$, of $1 \mathrm{~nm}$, a free energy barrier $\Delta G^{\ddagger}$ of $37 k_{B} T$, and a zero-force rate constant $k_{0}$ of $5 \times 10^{-21} \mathrm{ps}^{-1}$ (Table 4.3) and shown as solid line in Figure 4.15a). Finally, the parameters yielding the best fit were used to estimate the increase in the dissociation rate upon external force (black line in Figure 4.15b) according to Equation 1.3.
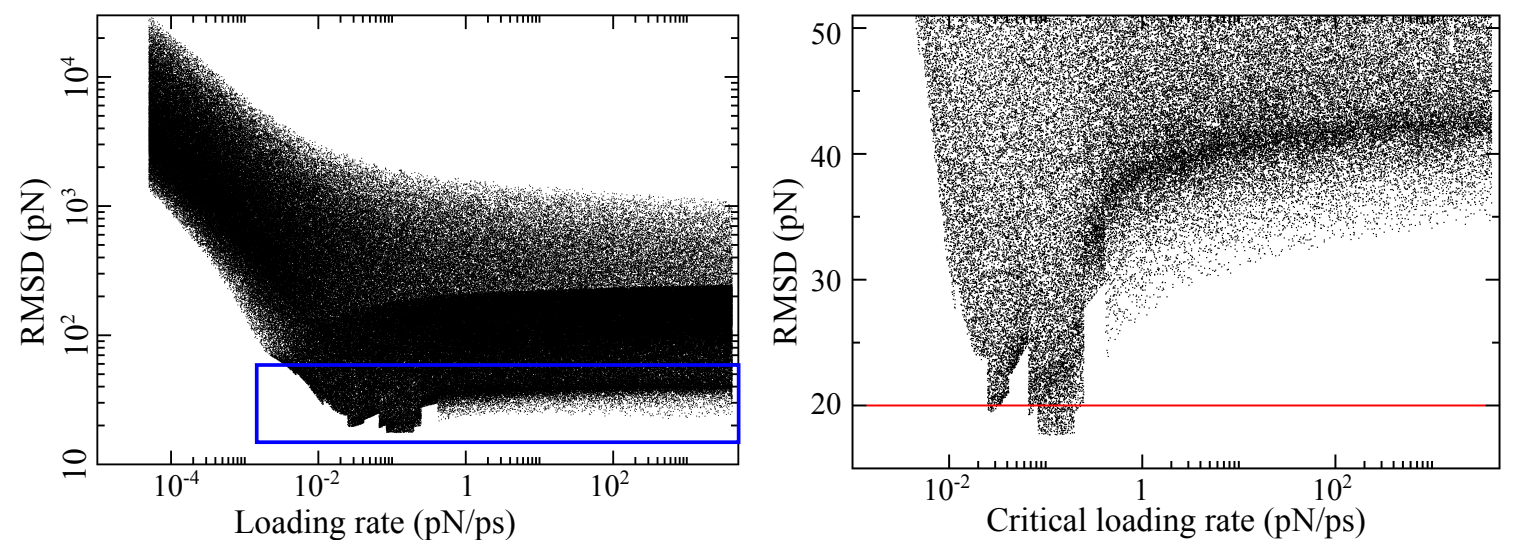

Figure 4.16 Uncertainty in the DHS fitting of rupture forces. (a) $500000 R M S D$ values were obtained from Monte Carlo sampling with random kinetic parameters $\Delta G, x$ and $L_{c r i t}$ as fitting parameters and projected as a function of critical loading rate. The blue square include all fits with an RMSD lower than the largest error $(50 \mathrm{pN})$ of the rupture forces from FPMD simulations. (b) Zoom of (a) (blue square), kinetic parameters with an $R M S D<20 \mathrm{pN}$ (red line) were used to quantify their mean and variations (Table 4.3 and Figure 4.17).

Given the large scattering of the rupture forces $(50 \mathrm{pN})$ in our limited sample of 43 events along 12 different loading rates, all fits with $R M S D<50 \mathrm{pN}$ were selected to analyze the uncertainty of fitting (labeled as blue square in Figure 4.16a). After zooming into the distribution of $R M S D$ along $L_{c r i t}$, three local minima could be observed (Figure 4.16b). However, as $L_{c r i t}$ is inversely proportional to $X_{b}$, the fits at the left local minimum with $L_{c r i t}<1 \times 10^{-4} \mathrm{pN} / \mathrm{ps}$ resulted in an unrealistic large $X_{b}(>1.4 \mathrm{~nm})$. Therefore, the fits 
with $R M S D<20 \mathrm{pN}$ (under the red line in Figure 4.16b) were selected to estimate the variability of our fitting and is shown as gray area in Figure 4.15. Figure 4.17 shows the respective distributions of parameters from fits with $R M S D<20 \mathrm{pN}$. The interquartile range (IQR) in the boxplot, representing the range of data between the upper quartile and lower quartile, was considered as the uncertainty of each parameter (Table 4.3).

Table 4.3 Kinetic parameters obtained from fitting the DHS model to the FPMD simulation data.

\begin{tabular}{c|c|c|c|c}
\hline $\operatorname{RMSD}(\mathrm{pN})$ & $k_{0}\left(\mathrm{ps}^{-1}\right)$ & $\Delta G^{\ddagger}\left(k_{B} T\right)$ & $\mathrm{X}_{b}(\mathrm{~nm})$ & $\mathrm{L}_{c r i t}\left(\mathrm{pN}^{2} \mathrm{ps}^{-1}\right)$ \\
\hline${ }^{*} 17.6$ & $5.3 \mathrm{e}-21$ & 37.0 & 1.0 & $7.5 \mathrm{e}-2$ \\
\hline${ }^{\dagger}<20$ & $7.3 \mathrm{e}-25-5.0 \mathrm{e}-20$ & $34.6-46.00$ & $0.94-1.30$ & $9.5 \mathrm{e}-2-1.5 \mathrm{e}-1$ \\
\hline
\end{tabular}

${ }^{*}$ Best fit with DHS model (see section 4.2.3)

${ }^{\dagger} \mathrm{IQR}$ in the boxplot of each kinetic parameters obtained from all fits with $\mathrm{RMSD}<20$.

Rupture forces from the FPMD simulations of membrane-bound-FAK with diagonal pulling (green) are consistent with the forces obtained from FAK-only FPMD simulations, whereas the forces resulting from the vertical pulling (magenta) are much lower and outside of the gray area. Vertical pulling results in a zipper-like opening, supporting the opening motion of FAK under force (Figure 4.10c). The primary force direction in the cellular context is unknown, and the FAK-only results are used in the following as an upper force limit.

The application of an external force leads to a lowering of the free energy barrier, resulting in a higher rate of the FERM-kinase domain dissociation. According to the relationship between $k_{f}$ and $k_{0}$ provided by the DHS model, we estimate that a force of around $\sim 20 \mathrm{pN}$ ) can accelerate the activation rate of FAK by more than 5 orders of magnitude (Figure $4.15 b$ ). 

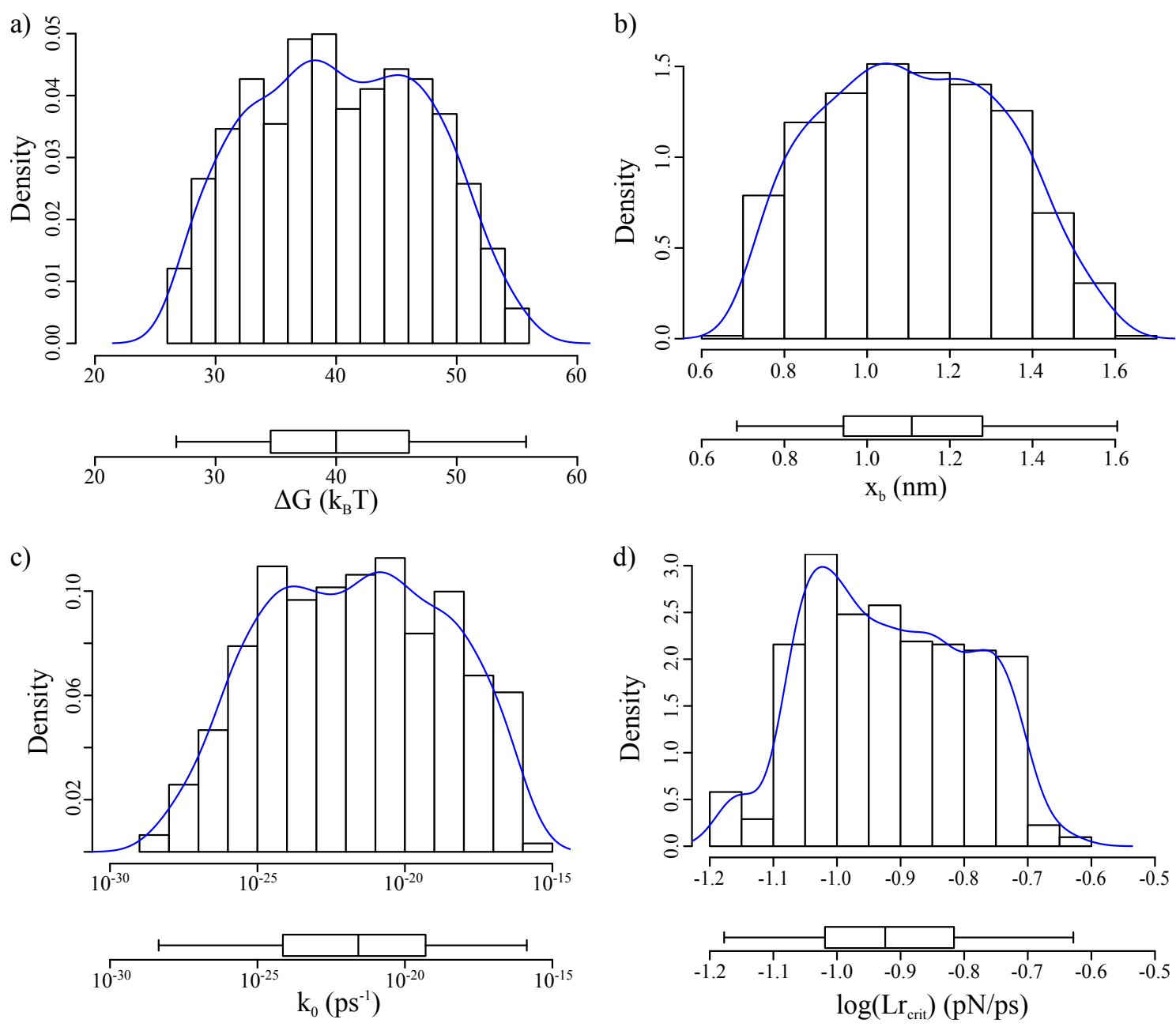

Figure 4.17 Uncertainty in the parameters from DHS fitting. Distributions of the estimated kinetic parameters obtained from the fits with $R M S D<20 \mathrm{pN}$, including $\Delta G$ (a), $x_{b}$ (b), $k_{0}$ (c), $L r_{c r i t}$ (d) are shown as histogram and boxplot. The interquartile range (IQR) in the boxplot, the range between upper quartile and lower quartile, is considered as erro bar of each parameter. 


\subsection{Kinetic model of FAK signaling.}

As illustrated in Figure 1.1 in Section 1.1, FAK acting as a cellular mechanosensor transduces mechanical signals from the cell surface to the nucleus. Previous studies reported that Ras activation regulated by FAK, Src and SHC is essential for the ERK activation [120, 121]. In the ERK/MAPK signaling cascade, signal transduction through FAK is achieved by multiple Grb2-mediated signaling pathways [121] (Figure 4.18): (1) The SH2 domain of She binds to the auto-phosphorylated tyrosine site Tyr397 in FAK, and then is trans-phosphorylated by FAK. Phosphorylated Shc provides the binding site for Grb2. Subsequently, the FAK-Shc-Grb2 complex associates with SOS and accelerates the exchange of GDP in Ras against GTP. (2) The $\mathrm{SH} 2$ domain of c-Src binds to the auto-phosphorylated tyrosine site Tyr397 in FAK and transphosphorylates Tyr925 in the FAT domain, which binds subsequently to Grb2. The FAK-Src-Grb2 complex associates then with SOS and contributes to the acceleration of the exchange of GDP/GTP in Ras protein. On the basis of these reaction steps, we established a kinetic model of a FAK-mediated signaling pathway, which contributes to the mechanical regulation of ERK activation (Figure 4.21).

The FAK-mediated signal transduction starts with the auto-phosphorylation of the tyrosine site of Tyr397 at the linker between the FAK FERM and kinase domain. Two mechanisms of FAK auto-phosphorylation have been suggested [122] (Figure 4.19): (1) Tyr397 undergoes intermolecular phosphorylation due to FAK dimerization or multimerization at the cell periphery (trans-phosphorylation), or (2) Tyr397 is able to access the active site of the same FAK protein (cis-phosphorylation). Phosphorylation, being if cis or trans, is re- 
quired to open FAK. Also, studies have showed that the auto-phosphorylation of Tyr397 is regulated by external force $[33,123,124]$. Thus, the opening motion of FAK is considered as the first reaction in our kinetic model. Since force also acts against a closing of the open state, and the subsequent immediate association with c-Src futher reduces the possibility of FAK closing, the opening motion of FAK is considered as an irreversible process and treated as a first order reaction. The force-dependent FAK FERM-kinase dissociation rate obtained from our FPMD simulations is used as a parameter to describe this reaction. Recent studies $[41,125]$ have shown that the clustering of FAK is critical for the auto-phosphorylation of Tyr397. Hence, the auto-phosphorylation reaction is treated as a Michaelis Menten mechanism with FAK as the enzyme for itself. However, the auto-phosphorylation rate of the tyrosine site in FAK is still unknown and redundant, the parameter of the tyrosine autophosphorylation in Src is used here.

(a)

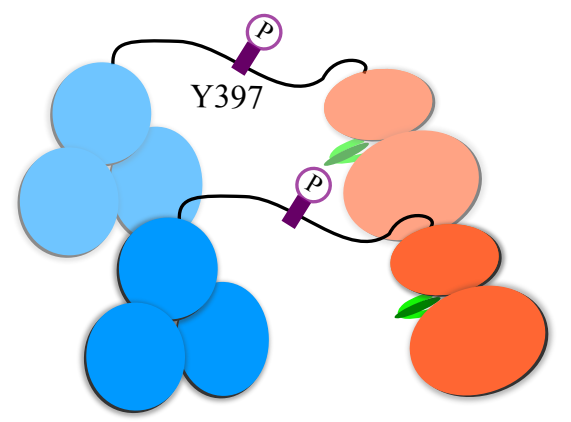

(b)

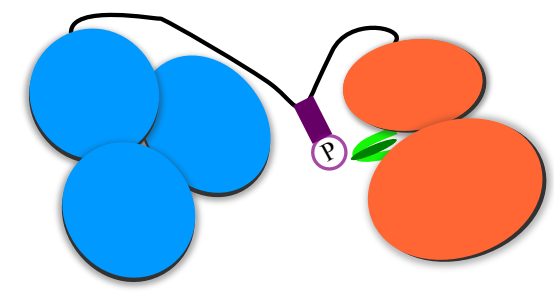

Figure 4.19 Schematic representation of two FAK auto-phosphorylation mechanisms: (a) FAK dimerization-induced intermolecular phosphorylation and (b) intramolecular phosphorylation of Tyr397. The dark green sticks show the localization of ATP in activation site.

The upstream mechanical activation of Src is not well understood yet, but experiments [126] have shown that the auto-phosphorylation of Tyr416 in Src is responsible for the activation of Src. Thus, the activation of Src is simplified in our model, and only the step of auto-phosphorylation at Tyr416 is considered. The downstream steps of our proposed kinetic model are based on two existing mathematical models. The first model, put forward by Kholodenko et al, describes the signal transduction mediated by the EGF receptor [127], which is constituted by similar interactions through two Grb2-mediated signaling pathways as FAK signaling (Figure 1.1). Except from the sequential phosphorylations in FAK (Tyr397, Try576/577 and Try925) after the force-induced domain opening, all parameters from Kholodenko's model were adopted to our model, including the thermodynamic restrictions along cyclic pathways in the reaction scheme (Figure 4.21), e.g. 
$k_{10} \cdot k_{14} \cdot k_{16} \cdot k_{18} / k_{-10} \cdot k_{-14} \cdot k_{-16} \cdot k_{-18}=1$. Parameters for the activation of the GDP/GTP exchange in Ras accelerated by FAK-Grb2-SOS complex is taken from Hatakeyama's study [128], which describes this process with an approximate Michaelis-Menten mechanism.
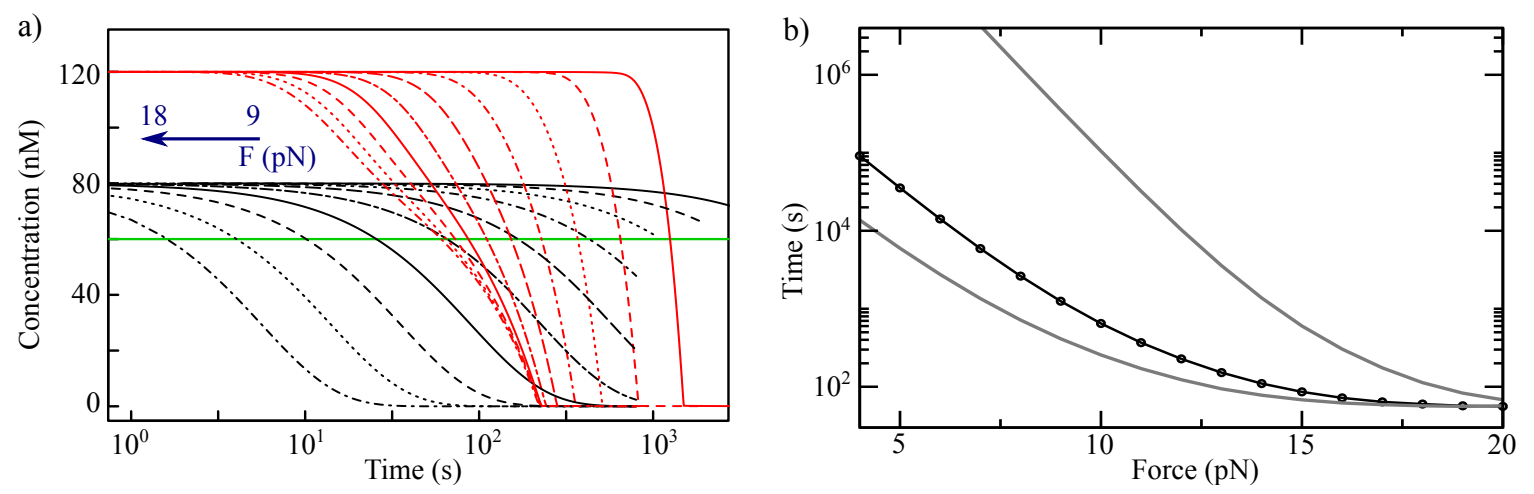

Figure 4.20 Results of downstream FAK signaling. (a) Time course of FAK opening (black) and the GDP/GTP exchange in Ras protein (red) obtained with a varying external force in range between 9 and $18 \mathrm{pN}$ (along the direction pointed by the blue arrow). Black: decrease in the concentration of closed FAK. Red: decrease in the concentration of GDP bound Ras protein. (b) Time at which $50 \%$ of GDP-bound Ras protein is consumed under varying external force, corresponding to the cross points of the green and red lines in (a). Black: data for kinetic parameters $k_{0}, \Delta G^{\ddagger}$ and $X_{b}$ of the best fit (Table 4.3). Gray: estimated upper and lower limits using the error ranges of the parameters (Table 4.3).

Based on the proposed kinetic model, we computed the time course of the responses to external forces. Figure $4.20 a$ illustrates how the total concentrations of FAK in the closed state (black lines) and of GDP bound Ras protein (red lines) reduced upon the external forces varying from 9 to $18 \mathrm{pN}$. Both FAK opening and GDP/GTP exchange in Ras are accelerated by the external force. For forces smaller than $15 \mathrm{pN}$, the increase in GDP/GTP exchange rate with force was strongly follows the one of FAK opening and thus is regulated by this force-dependent process (Figure 4.20a). In contrast, when the force was larger than $15 \mathrm{pN}$, the GDP/GTP exchange does not further accelerate, but instead saturates, in contrast to the steadily increase FAK opening rate.

To trace this force-dependent regulation of Ras activation more precisely, the time when $50 \%$ of GDP in Ras proteins were exchanged against GTP are shown as a function of external force in Figure $4.20 b$ (black line). With a small external force $(<5 \mathrm{pN})$, Ras activation cannot occur within one day, but occurs within seconds to minutes at intermediate forces. The activation rate of Ras then remained stable when further increasing the external force ( $>15 \mathrm{pN}$ ). The ranges of estimated errors of $k_{0}, \Delta G^{\ddagger}$ and $X_{b}$ (Table 4.3) were used to predict the variation of the mechanical regulation of Ras activation. The upper limit 
(upper gray line in Figure 4.20b) was predicted with the largest $k_{0}$ and the smallest $\Delta G^{\ddagger}$ and $X_{b}$, which represented the lowest estimated energy barrier of FAK opening motion. The smallest $k_{0}$ and the largest $\Delta G^{\ddagger}$ and $X_{b}$ were used to predicted the lower limit (lower gray line), which corresponded to the highest estimated energy barrier between FAK closed and opened states. While the Ras GDP/GTP exchange rate varies strongly (by several orders of magnitude) within the parameter uncertainty, the increase in rate toward a second scale is reached in all cases at around $20 \mathrm{pN}$.

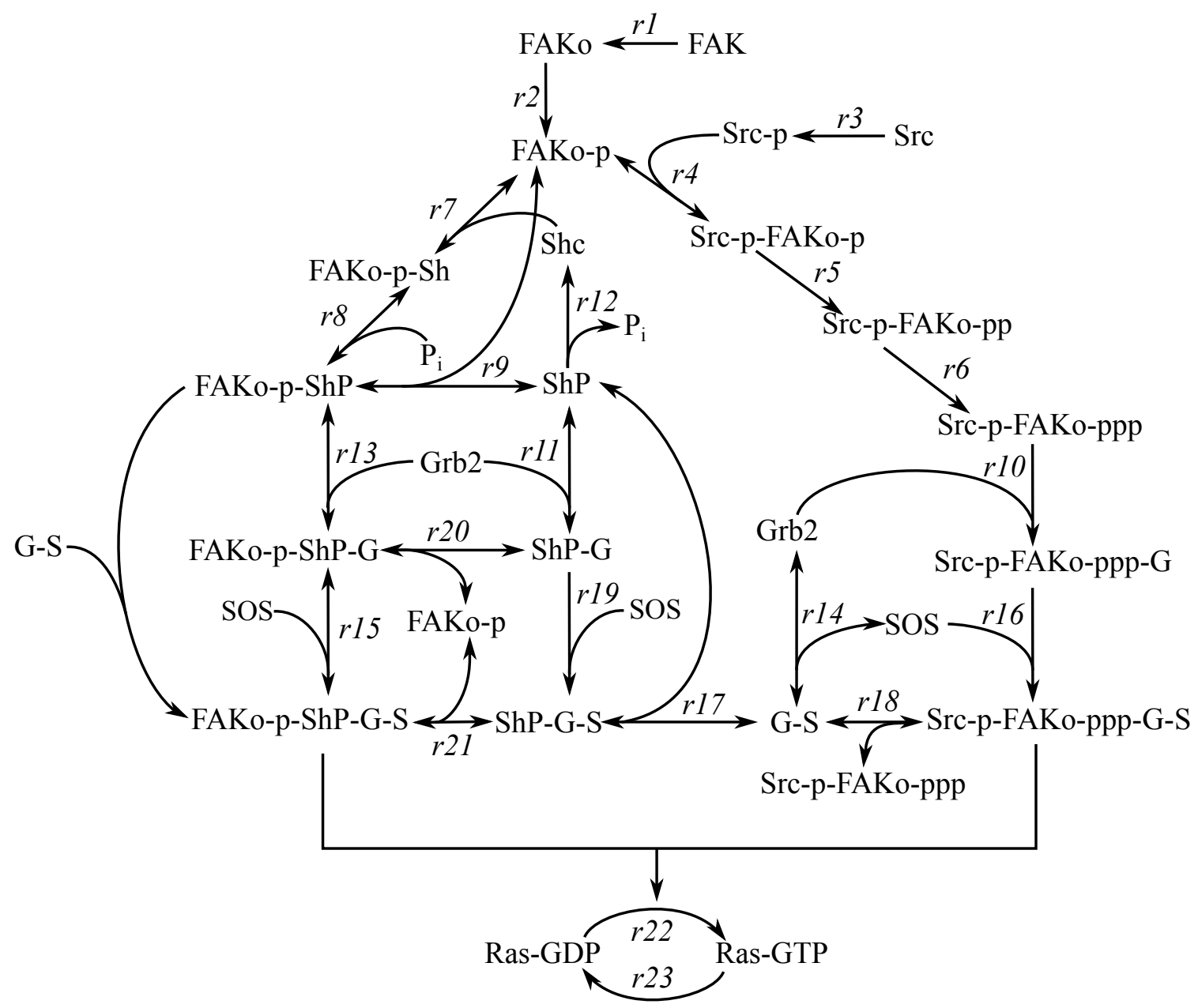

Figure 4.21 Kinetic scheme of FAK signaling regulated by external force. Numbering of individual reactions is arbitrary. 


\subsection{Kinetic model of FAK signaling.}

Table 4.4 List of parameters for FAK signaling model.

\begin{tabular}{|c|c|c|c|c|}
\hline & Units & Model value & Ref. value & Ref. \\
\hline$k_{01}$ & $\mathrm{~s}^{-1}$ & $5.3 e-9$ & - & - \\
\hline $\mathrm{F}^{*}$ & $\mathrm{pN}$ & $0 \sim 50$ & - & - \\
\hline$X_{b}^{*}$ & $\mathrm{~nm}$ & 1.00 & - & - \\
\hline$\Delta G^{* *}$ & $k_{B} T$ & 37 & - & - \\
\hline$k_{02 c a t}$ & $\mathrm{~s}^{-1}$ & 9.33 & $560 \min ^{-1}$ & [126] \\
\hline$k_{02 m}$ & $\mathrm{nM}$ & 0.132 & 0.132 & [126] \\
\hline$k_{03 \mathrm{cat}}$ & $\mathrm{s}^{-1}$ & 9.33 & $560 \min ^{-1}$ & [126] \\
\hline$k_{03 m}$ & $\mathrm{nM}$ & 0.132 & 0.132 & [126] \\
\hline$k_{04}$ & $\mathrm{nM}^{-1} \mathrm{~s}^{-1}$ & 0.09 & 0.09 & [127] \\
\hline$k_{04 b}$ & $\mathrm{~s}^{-1}$ & 0.6 & 0.6 & [127] \\
\hline$k_{05}$ & $\mathrm{~s}^{-1}$ & 9.33 & 9.33 & [126] \\
\hline$k_{06}$ & $\mathrm{~s}^{-1}$ & 9.33 & 9.33 & [126] \\
\hline$k_{07}$ & $\mathrm{nM}^{-1} \mathrm{~s}^{-1}$ & 0.09 & 0.09 & [127] \\
\hline$k_{07 b}$ & $\mathrm{~s}^{-1}$ & 0.6 & 0.6 & [128] \\
\hline$k_{08}$ & $\mathrm{nM}^{-1} \mathrm{~s}^{-1}$ & 6 & 6 & [127] \\
\hline$k_{08 b}$ & $\mathrm{~s}^{-1}$ & 0.06 & 0.06 & [127] \\
\hline$k_{09}$ & $\mathrm{~s}^{-1}$ & 0.3 & 0.3 & [127] \\
\hline$k_{09 b}$ & $\mathrm{nM}^{-1} \mathrm{~s}^{-1}$ & 0.0009 & 0.0009 & [127] \\
\hline$k_{10}$ & $\mathrm{nM}^{-1} \mathrm{~s}^{-1}$ & 0.003 & 0.003 & [127] \\
\hline$k_{10 b}$ & $\mathrm{~s}^{-1}$ & 0.05 & 0.05 & [127] \\
\hline$k_{11}$ & $\mathrm{nM}^{-1} \mathrm{~s}^{-1}$ & 0.003 & 0.003 & [127] \\
\hline$k_{11 b}$ & $\mathrm{~s}^{-1}$ & 0.1 & 0.1 & [127] \\
\hline$k_{12 m}$ & $\mathrm{nM}$ & 340 & 340 & [127] \\
\hline$V_{12 \max }$ & $\mathrm{nMs}^{-1}$ & 1.7 & 1.7 & [127] \\
\hline$k_{13}$ & $\mathrm{nM}^{-1} \mathrm{~s}^{-1}$ & 0.003 & 0.003 & [127] \\
\hline$k_{13 b}$ & $\mathrm{~s}^{-1}$ & 0.1 & 0.1 & [127] \\
\hline$k_{14}$ & $\mathrm{nM}^{-1} \mathrm{~s}^{-1}$ & 0.0015 & 0.0015 & [127] \\
\hline$k_{14 b}$ & $\mathrm{~s}^{-1}$ & 0.0001 & 0.0001 & [127] \\
\hline$k_{15}$ & $\mathrm{nM}^{-1} \mathrm{~s}^{-1}$ & 0.03 & 0.03 & [127] \\
\hline$k_{15 b}$ & $\mathrm{~s}^{-1}$ & 0.064 & 0.064 & [127] \\
\hline$k_{16}$ & $\mathrm{nM}^{-1} \mathrm{~s}^{-1}$ & 0.01 & 0.01 & [127] \\
\hline$k_{16 b}$ & $\mathrm{~s}^{-1}$ & 0.06 & 0.06 & [127] \\
\hline
\end{tabular}




\begin{tabular}{c|l|r|r|r}
\hline$k_{17}$ & $\mathrm{nM}^{-1} \mathrm{~s}^{-1}$ & 0.021 & 0.021 & {$[127]$} \\
\hline$k_{17}$ & $\mathrm{~s}^{-1}$ & 0.1 & 0.1 & {$[127]$} \\
\hline$k_{18}$ & $\mathrm{nM}^{-1} \mathrm{~s}^{-1}$ & 0.0045 & 0.0045 & {$[127]$} \\
\hline$k_{18 b}$ & $\mathrm{~s}^{-1}$ & 0.03 & 0.03 & {$[127]$} \\
\hline$k_{19}$ & $\mathrm{nM}^{-1} \mathrm{~s}^{-1}$ & 0.01 & 0.01 & {$[127]$} \\
\hline$k_{19 b}$ & $\mathrm{~s}^{-1}$ & 0.0214 & 0.0214 & {$[127]$} \\
\hline$k_{20}$ & $\mathrm{nM}^{-1} \mathrm{~s}^{-1}$ & 0.009 & 0.009 & {$[127]$} \\
\hline$k_{20 b}$ & $\mathrm{~s}^{-1}$ & 0.0429 & 0.0429 & {$[127]$} \\
\hline$k_{21}$ & $\mathrm{nM}^{-1} \mathrm{~s}^{-1}$ & 0.12 & 0.12 & {$[127]$} \\
\hline$k_{21 b}$ & $\mathrm{~s}^{-1}$ & 0.000214 & 0.000214 & {$[127]$} \\
\hline$k_{22}$ & $\mathrm{~s}^{-1}$ & 0.222 & 0.222 & {$[128]$} \\
\hline$k_{22 m}$ & $\mathrm{nM}$ & 0.181 & 0.181 & {$[128]$} \\
\hline$k_{22 m}$ & $\mathrm{nM}$ & 0.0571 & 0.0571 & {$[128]$} \\
\hline$V_{22 \max }$ & $\mathrm{nMs}$ & 0.289 & 0.289 & {$[128]$} \\
\hline
\end{tabular}




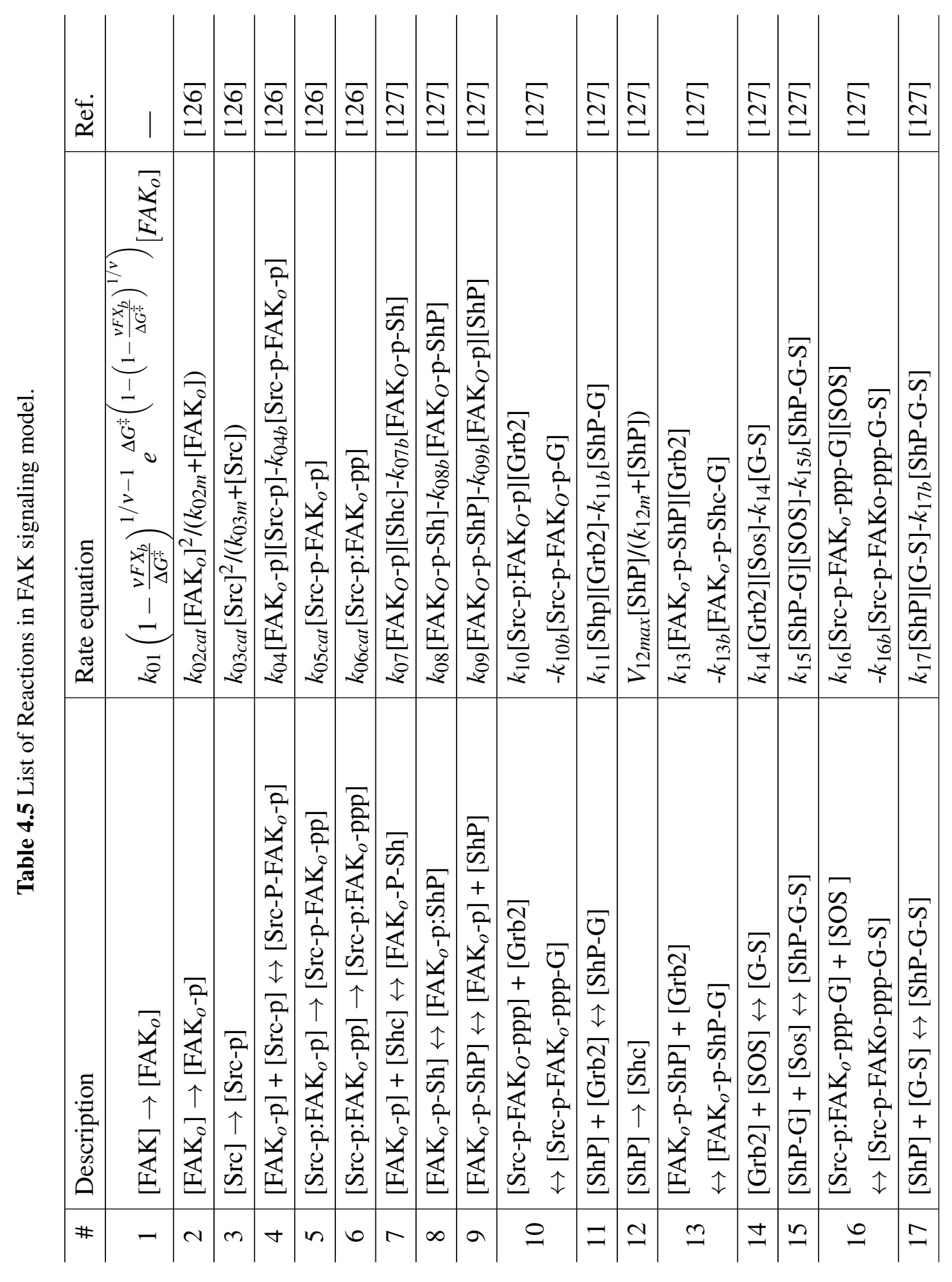




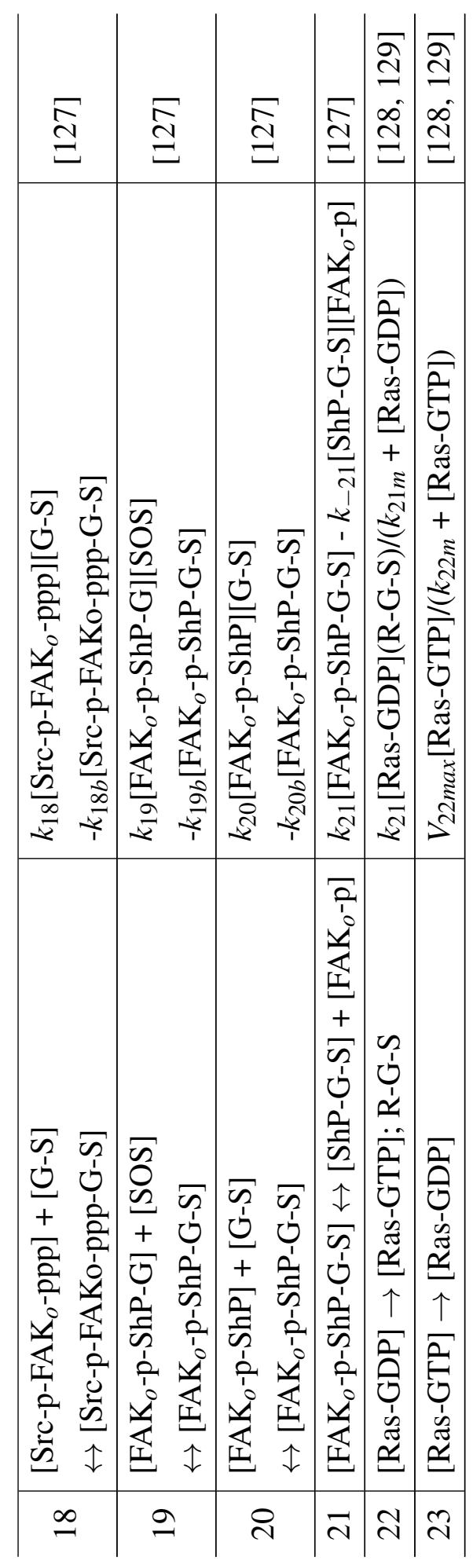


Table 4.6 List of species in FAK signaling model.

\begin{tabular}{|c|c|c|c|}
\hline Species & Name & $\begin{array}{r}\text { Initinal } \\
\text { Concentration }\end{array}$ & Ref. \\
\hline FAK & Focal adhesion kinase & $80 \mathrm{nM}$ & {$[129,130]$} \\
\hline $\mathrm{FAK}_{o}$ & Opened FAK & $0 \mathrm{nM}$ & - \\
\hline $\mathrm{FAK}_{o}-\mathrm{p}$ & Opened FAK-py397 & $0 \mathrm{nM}$ & - \\
\hline Src & Src kinase & $90 \mathrm{nM}$ & {$[129,130]$} \\
\hline Src-p & Auto-phosphorylated Src-py416 & $0 \mathrm{nM}$ & - \\
\hline Shc & Shc adaptor protein & $1000 \mathrm{nM}$ & {$[128,129]$} \\
\hline Shc-P & phosphorylated Shc & $0 \mathrm{nM}$ & - \\
\hline Grb2 & $\begin{array}{l}\text { Growth factor receptor } \\
\text { bound protein } 2\end{array}$ & $48 \mathrm{nM}$ & [130] \\
\hline ShP-G & Protein complex & $0 \mathrm{nM}$ & - \\
\hline SOS & Son of Sevenless & $62 \mathrm{nM}$ & {$[130]$} \\
\hline G-S & Grb2:SOS adaptor protein & $0 \mathrm{nM}$ & - \\
\hline ShP-G-S & Protein complex & $0 \mathrm{nM}$ & \\
\hline Src-p-FAK ${ }_{o}-\mathrm{p}$ & Protein complex & $0 \mathrm{nM}$ & - \\
\hline Src-p-FAK ${ }_{o}-\mathrm{pp}$ & $\begin{array}{l}\text { Protein complex } \\
\text { (FAK-py397-py576/577) }\end{array}$ & $0 \mathrm{nM}$ & - \\
\hline Src-p-FAK ${ }_{o}-\mathrm{ppp}$ & $\begin{array}{l}\text { Protein complex } \\
\text { (FAK-py397-py576/577-py925) }\end{array}$ & $0 \mathrm{nM}$ & - \\
\hline Src-p-FAK ${ }_{o}$-ppp-G & Protein complex & $0 \mathrm{nM}$ & - \\
\hline Src-p-FAK ${ }_{o}-$ ppp-G-S & Protein complex & $0 \mathrm{nM}$ & - \\
\hline $\mathrm{FAK}_{o}-\mathrm{p}-\mathrm{Sh}$ & Protein complex & $0 \mathrm{nM}$ & - \\
\hline $\mathrm{FAK}_{o}-\mathrm{p}-\mathrm{ShP}$ & Protein complex & $0 \mathrm{nM}$ & - \\
\hline FAK ${ }_{o}-\mathrm{p}-\mathrm{ShP}-\mathrm{G}$ & Protein complex & $0 \mathrm{nM}$ & - \\
\hline $\mathrm{FAK}_{o}-\mathrm{p}-\mathrm{ShP}-\mathrm{G}-\mathrm{S}$ & Protein complex & $0 \mathrm{nM}$ & - \\
\hline Ras-GDP & Ras protein (GDP state) & $120 \mathrm{nM}$ & {$[128,129]$} \\
\hline Ras-GTP & Ras protein (GTP state) & $0 \mathrm{nM}$ & - \\
\hline R-G-S & $\begin{array}{l}\text { Protein complex containing } \\
\text { Grb2:SOS }\end{array}$ & $0 \mathrm{nM}$ & - \\
\hline
\end{tabular}




\subsection{Discussion and Conclusion}

We here provide computational evidence for a force-induced activation pathway of FAK. It is non-trivial that the exertion of a pulling force at opposite sites of the FERM and kinase domains leads to their dissociation. Other likely scenarios are protein unfolding and $\mathrm{PIP}_{2}$-protein dissociation, both of which would lead to FAK deactivation, as obviously an intact kinase structure but also $\mathrm{PIP}_{2}$ binding [41] are required for FAK activity. We here find domain-domain dissociation to be strongly preferred over unfolding or membrane detachment, over a large range of pulling velocities, and robust with regard to the membrane attachment, decidedly arguing for a mechano-sensing function of FAK.

Force application sites in FAK are critical for the FERM-kinase domain opening. Applying a stretching force to FERM F1- or F3-lobe leads to a preference of FAK deactivation through kinase unfolding over its activation through FERM-kinase dissociation. In contrast, applying mechanical force on the basic patch in FERM and C-terminus of kinase domain results in domain dissociation with minor structural unfolding, which strikingly emphasizes the crucial role of positively charged basic patch in the force transduction through FAK.

$\alpha$-helical proteins are known to unfold at forces typically lower than $\beta$-sheet proteins[131], and both the kinase C-lobe and the FERM F2 domain feature mainly $\alpha$-helical secondary structure. In this regard, force-induced FAK unfolding would be an expected result. Nevertheless, in the particular and physiologically relevant case that force is applied to the $\mathrm{PIP}_{2}$ binding site and the kinase $\mathrm{C}$-terminus, the $\alpha$-helical regions subjected to the pulling force refrain from unfolding. They instead transduce the load to the F2/C-lobe interface, which readily opens prior to substantial kinase unfolding. We suggest that it is the zipper-like topology, with the force application sites both located at the membrane-proximal basis of the two domains, that mechanically weakens the domain interface, resulting in efficient FAK opening and activation. Force transduction through the two termini, in contrast, results in shearing the two domains relative to each other, making them less prone to dissociate. The lower mechanical resistance of zipper versus shear-type topologies has been described earlier, primarily for $\beta$-sheets [46, 47], and FAK force-induced domain-domain rupture and activation appears to be another variation of this theme.

The interaction at the domain interface is highly dynamic. Especially during the unfolding process, the interface residues repeatedly exchange interaction partners. TRFDA 
has been shown earlier in this study to be an efficient computational approach to track protein allostery even with minor conformational changes (Section 4.6.2). Here, by using TRFDA, residues playing essential roles for protein mechanical stability can be identified. This helped us to predict interesting candidates for functional mutagenesis. We here suggest D200 and R598, as such candidates mutagenesis. They contribute together with Y180, M183, V196 and F596, which previously have been shown to stabilize the auto-inhibited state [36], to the rupture force and their mutation is expected to result in increased FAK activity, according to our results.

By performing extensive FPMD simulations in presence of the POPE membrane bilayer with varying $\mathrm{PIP}_{2}$ concentration, we tested our proposed model of FAK mechano-sensing. In this model, tensile force propagates from the membrane through the $\mathrm{PIP}_{2}$ binding site of the FERM domain and from the cytoskeleton-anchored FAT domain. They activate FAK by relieving the occlusion of the central phosphorylation site of FAK (Tyr576/577) by the autoinhibitory FERM domain. We observed that the dissociation of the kinase domain from the membrane and the FERM domain induced by an external force applied on the C-terminal of kinase domain is independent of the force application directions. However, it is dependent on the concentration of $\mathrm{PIP}_{2}$ in the membrane. The interactions of FERM domain with a single PIP 2 lipid is insufficient to hold FAK until the dissociation between FERM and kinase domain occurs, i.e. only aggregated PIP $_{2}$ lipids in the membrane can trigger the mechanical activation of FAK.

Using an advanced dynamic force spectroscopy model by Dudko, Humer, and Szabo, the kinetic parameters, which describes the energy landscape of the transition of FAK from the closed to the opened state, were estimated (Section 4.7). However, the rupture forces from our simulations show a large variations, which increases the uncertainty of the predicted kinetic parameters. The large scattering of the obtained forces is due to the limitation of computer resources. Increasing the sampling and extending it to even lower loading rates can effectively minimize the error of the extrapolated parameters.

On the basis of the interactions in the FAK-mediated downstream signaling pathway, we established a kinetic model to analyze the enzymatic effect of FAK on Ras activation, which regulates the gene expression via ERK activation (Section 4.8). The proposed kinetic model is based on two previously established mathematical models. However, the upstream mechanical activation of FAK has so for been missing. Our results in this work can fill this gap, which is still inaccessible to the experiment yet, and bring the factor of force into 
the regulation of downstream reactions. We observe that external forces within $20 \mathrm{pN}$ can significantly accelerate the activation of Ras, whereas a larger external force $(>20 \mathrm{pN})$ does not further promote the GDP/GTP exchange process in Ras. We thus overall observe two force regimes. Firstly, within $50 \mathrm{pN}$ or less, the activation of FAK can be increased about ten orders of magnitude (Figure 4.15b), i.e. the time scale of FAK activation comes down to a few seconds, and secondly, only external forces within $20 \mathrm{pN}$ contribute to the acceleration of Ras activation (Figure 4.20b). Recent experiments [132] showed that the tensile force acting on integrin lies mostly in the range of 33 to $43 \mathrm{pN}$, which is in good agreement with our computational results. Further more direct evidence of the force levels on FAK and downstream effects are needed to test our computers predictions. 


\section{CHAPTER 5}

\section{CONCLUSIONS AND FUTURE PERSPECTIVES}

An increasing number of mechano-sensing proteins are gaining attention due to their crucial role in the conversion of external forces from extracellular matrix into intracellular biochemical reactions. FAK is recognized as force sensor at the cytoplasmic site of cell membrane. Multiple studies have shown that the malfunction of FAK can lead to errors in embryonic development and survival [28-30]. Thus, understanding the mechanism of FAK mediated signaling is helpful for the development of treatments for diverse human diseases such as cancer or cardiovascular diseases. Here, we addressed two major questions: First, how does FAK respond to an external biochemical (ligand binding) or mechanical (force) signal? Second, how does FAK as a mechano-sensor affect the regulation of downstream signaling?

To answer the first question above, the dynamics of FK-FAK in three protein systems in absence (apo-FK) or presence of ATP (FK-ATP) and additional PIP 2 (FK-ATP-PIP 2 ) were compared by performing all-atom MD simulations (Chapter 3). We identified an opening motion between the FERM and kinase domains (upon ATP binding) and a reversible closing (upon ATP binding) and opening (upon additional $\mathrm{PIP}_{2}$ ) motion within the kinase domain, which is in good agreement with FRET experiment. However, no full dissociation as required for FAK catalytic turnover, was observed between the FERM and kinase domains [41]. This result hints towards a requirement of an additional biochemical or mechanical stimuli for FAK activation. Accordingly, a model of FAK mechanical activation was proposed and tested by applying an external force on FK-FAK in FPMD simulations, which is meant to mimic the propagation of tensile force through FAK (Chapter 4). We 
observed a specific opening between the FERM and kinase domains, however only when applying force on the basic patch in the FERM domain and C-terminus of the kinase domain. This strongly underlines that the interactions of basic patch with its binding partner is crucial for FAK activation.

To validate this mechano-activation mechanism in the more physiological context of membrane-bound FAK, protein systems with membranes containing different $\mathrm{PIP}_{2}$ concentrations were built and FK-FAK kinase C-terminus was pulled away from the membrane. The simulation data showed force-induced activation, i.e. dissociation of the kinase domain from the FERM domain prior to the dissociation of the protein from membrane at a high concentration of $\mathrm{PIP}_{2}(15 \%)$ in the membrane. In contrast, a membrane with low concentration of $\mathrm{PIP}_{2}(1 \%)$, in which the FERM domain interacts with a single $\mathrm{PIP}_{2}$, cannot hold FAK until the dissociation between FERM and kinase domains occurs. We can conclude that $\mathrm{PIP}_{2}$-clustering is a prerequisite for FAK mechanical activation.

To answer our second major question on downstream consequences of FAK mechanosensing, a signaling network connecting the initial opening of FAK by force to downstream Ras activation was established. Force-dependent FAK activation turns also Ras GDP/GTP exchange along with further downstream ERK/MAPK signaling into a force-regulated process. Our kinetic model provides straightforward insight into the enzymatic role of FAK in the signal transduction from the cell surface to the nucleus. A major finding is that force leads to a steady acceleration of FAK activity, which translates into an acceleration of RasGTP formation only up to a certain force threshold, estimated to lie in the $15-25 \mathrm{pN}$ range, this has interesting implications for the role of FAK as mechano-sensor in the cell. To date, many kinetic models of the MAPK signaling pathway have been suggested [128, 133] which solely focus on the chemical triggering of the MAPK signaling, namely by epidermal growth factors as stimuli. However, the mechanical signaling along the MAPK pathway has not yet been investigated as parameters on upstream mechanical activation reactions. We here fill this gap and for the first time, to our knowledge, propose a mechano-biochemical combined signaling pathway, in which the first step is triggered by force. Introducing our proposed activation mechanism of FAK into the downstream signaling network allows to predict ERK activation kinetics as a function of external force, a dependency already observed previously in multiple experiments $[123,134]$.

Given the limitation of computational resources, a large scattering of rupture forces with varying loading rates was obtained in our simulations, which can lead to errors in the 
estimation of kinetic parameters that describe the energy landscape of the transition from the closed to the opened state. To minimize the uncertainties in the rupture forces and thereby in the extrapolated parameters, one would need to increase the sampling at each loading rates or to extend the simulations to millisecond time scales. Simulations at millisecond time scales have come into reach, in particular on hardware optimized for MD simulations. [135] A combination of all-atom simulations as presented here with simulations of more coarse-grained models can additionally extend the accessible time scales. Most notably, the Self-Organized Polymer (SOP) model has proven useful in a series of simulations of force-induced protein unfolding or dissociation such as ubiquitin reversible unfolding [136]. In the case of FAK, it remains to be seen, however, if the delicate balance between domain-domain dissociation and domain unfolding of FAK is correctly captured by a model such as the SOP model, in which all residue-residue interactions are treated equally strong.

Due to the computational cost of considering all atomistic details, the loading rates used in our computational force-spectroscopy like simulations were four to six orders of magnitude faster than those used in experimental force-spectroscopy measurements. The DHS model allowed to extrapolate to longer time scale, though with high uncertainty, as discussed above. Also, two regimes separated by the critical loading rate needed to be considered in our fitting with DHS model. Recently, a theory of rapid force spectroscopy has been suggested by Bullerjahn et al. [137], which can be used to fit force spectroscopy data at a large range of time scales including those of our simulations (up to $10^{11} \mathrm{pN} / \mathrm{s}$ ). Applying this new model to our data will be a subject of future work on FAK.

Interestingly, FAK is not the only upstream signaling protein, which has an auto-inhibitory conformation and is regulated by force. Src is another well-studied example which responds to cellular stresses, presumably by releasing its kinase domain from the interactions with its inhibitory SH2 and SH3 domains [138]. The binding of Src to FAK induces one of the FAK mediated signaling pathways leading to Ras activation. Thus, the strongly simplified activation of Src in our proposed model, in which only the step of auto-phosphorylation is considered, could lead to an overestimation of the Src activation rate. Thus, studying Src mechanical activation using FPMD simulations as presented in this thesis for FAK would allow to analyze the role of Src as a mechano-enzyme. Such results could also improve the accuracy of our predictions of the mechanical regulation of the ERK/MAPK signaling pathway. However, auto-inhibited proteins such as FAK and Src at membrane surface are numerous. Other candidates, e.g. ERM, SNARE and WASP proteins, are also well known 
for their auto-inhibitory conformations [138] and their role in the regulation of downstream signaling. Extending our computational analyses systematically to a wider range of autoinhibited membrane proteins might help to explore how widespread mechanical regulation at the membrane surface is.

We identified a crucial role of $\mathrm{PIP}_{2}$-clustering and force propagation through the $\mathrm{PIP}_{2}$ enriched membrane for FAK-mediated signaling by our MD simulations. This might be a common theme also for other mechano-transduction mechanisms. The supposedly multivalent binding of FAK to $\mathrm{PIP}_{2}$ as suggested by our results have yet to be confirmed by experiments. Surface Plasmon Resonance (SPR) might be a candidate to more quantitatively assess the binding of FAK with $\mathrm{PIP}_{2}$ micro-domains. As a further test of our predictions, it remains to be investigated experimentally to what extent FAK mechanical activation depends on calcium ions, which can enhance $\mathrm{PIP}_{2}$ clustering.

Two force regimes have been observed in our study. First, within $40 \mathrm{pN}$, the activation of FAK can be increased by 5-10 orders of magnitude; second, within $20 \mathrm{pN}$, the activation of Ras is accelerated with increasing of external force. The force acting on FAK at FAs is still unknown. For other proteins at FAs, previous studies reported that the unfolding rate of a talin rod can reach a time scale of seconds with an external force of $\sim 12 \mathrm{pN}$ measured by magnetic tweezers [94]. Grashoff et al. [95] could quantify the tension across single vinculin molecules in FAs to be approximately $2.5 \mathrm{pN}$, using a FRET-based mechanosensor. A recent study by Wang et al. [132] demonstrated that the tensile force acting on single integrin-ligand interactions at the cell surface is about $40 \mathrm{pN}$, which is in good agreement with our computational result. To further validate the predicted molecular mechanism of FAK activation, experiments applying a stretching force on single FAK protein are required. We cooperate with Hermann Gaub's lab in Munich to test FAK mechanical unfolding with atomic force microscopy experiments. The unfolding from the $\mathrm{N}$ - and $\mathrm{C}$-terminus of the FK-FAK fragment can be easily achieved by attaching the cantilever to the termini through a biochemical handle. These experiments have yielded preliminary data, that largely is in line with our observations. However, the catalytic turnover of FAK requires the force to be applied to the FERM basic patch and the C-terminus of the kinase domain, which is much more challenging, because a loop carrying a covalent handle is required to be inserted within the basic patch. Also, cyclic stretch experiments would be of interest to test the enzymatic effect of FAK for the GDP/GTP exchange in Ras, as predicted in our proposed signaling network. It would be also of high interest to quantify the force transmitted through FAK within FAs using the FRET-based sensor of Grashoff and co-workers. 
Taken together, our Molecular Dynamics simulations provided evidence on the molecular level for that FAK can be allosterically regulated both by biochemical and mechanical stimuli or rather a combination thereof. Biochemical modeling and in general systems biology approaches can be extended to incorporate mechanical, a crucial biological stimulus that remains to be often overlooked in this field. Our hybrid approach of molecular simulations and biochemical network modeling, combining a biophysical and biochemical perspective of a cellular process, is likely to be useful for studying other potential cellular or extra-cellular mechano-enzymes. 



\section{REFERENCES}

[1] D. T. Butcher, T. Alliston, and V. M. Weaver. A tense situation: forcing tumour progression. Nat. Rev. Cancer, 9(2):108-22, 2009.

[2] V. Vogel and M. Sheetz. Local force and geometry sensing regulate cell functions. Nat. Rev. Mol. Cell Biol., 7(4):265-275, 2006.

[3] C. E. Sarraf, W. R. Otto, and M. Eastwood. In vitro mesenchymal stem cell differentiation after mechanical stimulation. Cell Prolif., 44(1):99-108, 2011.

[4] A. D. Bershadsky, N. Q. Balaban, and B. Geiger. Adhesion-dependent cell mechanosensitivity. Annu. Rev. Cell Dev. Biol., 19:677-95, 2003.

[5] J. Zhu, B. H. Luo, T. Xiao, C. Zhang, N. Nishida, and T. A. Springer. Structure of a complete integrin ectodomain in a physiologic resting state and activation and deactivation by applied forces. Mol. Cell, 32(6):849-61, 2008.

[6] V. P. Hytönen and V. Vogel. How force might activate talin's vinculin binding sites: Smd reveals a structural mechanism. PLoS Comput. Biol., 4(2):e24, 2008.

[7] M. Yao, B. T. Goult, H. Chen, P. Cong, M. P. Sheetz, and J. Yan. Mechanical activation of vinculin binding to talin locks talin in an unfolded conformation. Sci. Rep., 4:4610, 2014.

[8] C. Ciobanasu, B. Faivre, and C. Le Clainche. Actomyosin-dependent formation of the mechanosensitive talin-vinculin complex reinforces actin anchoring. Nat. Commun., 5:3095, 2014.

[9] S. E. Lee, R. D. Kamm, and M. R. Mofrad. Force-induced activation of talin and its possible role in focal adhesion mechanotransduction. J. Biomech., 40(9):2096-2016, 2007.

[10] AW. Holle, X. Tang, D. Vijayraghavan, LG. Vincent, A. Fuhrmann, YS. Choi, JC. del Álamo, and AJ. Engler. In situ mechanotransduction via vinculin regulates stem cell differentiation. Stem Cells, 31(11):2467-2477, 2013.

[11] J. Golji and M. R. Mofrad. A molecular dynamics investigation of vinculin activation. Biophys. J., 99(4):1073-1081, 2010. 
[12] X. Zhang, S. W. Moore, T. Iskratsch, and M. P. Sheetz. N-wasp-directed actin polymerization activates cas phosphorylation and lamellipodium spreading. J. Cell Sci., 127(Pt 7):1394-1405, 2014.

[13] Y. Sawada, M. Tamada, B. J. Dubin-Thaler, O. Cherniavskaya, R. Sakai, S. Tanaka, and M. P. Sheetz. Force sensing by mechanical extension of the src family kinase substrate p130cas. Cell, 127(5):1015-26, 2006.

[14] P. Kanchanawong, G. Shtengel, A. M. Pasapera, E. B. Ramko, M. W. Davidson, H. F. Hess, and C. M. Waterman. Nanoscale architecture of integrin-based cell adhesions. Nature, 468(7323):580-584, 2010.

[15] R. W. Carpick and M. Salmeron. Scratching the surface: Fundamental investigations of tribology with atomic force microscopy. Chem. Rev., 97(4):1163-1194, 1997.

[16] B. Jagannathan and S. Marqusee. Protein folding and unfolding under force. Biopolymers, 99(11):860-869, 2013.

[17] C. Gosse and V. Croquette. Magnetic tweezers: micromanipulation and force measurement at the molecular level. Biophys. J., 82(6):3314-3329, 2002.

[18] A. Kishino and T. Yanagida. Force measurements by micromanipulation of a single actin filament by glass needles. Nature, 334(6177):74-76, 1988.

[19] E. Evans, K. Ritchie, and R. Merkel. Sensitive force technique to probe molecular adhesion and structural linkages at biological interfaces. Biophys. J., 68(6):2580 2587, 1995.

[20] R. Jr. Roskoski. Src kinase regulation by phosphorylation and dephosphorylation. Biochem. Biophys. Res. Commun., 331(1):1-14, 2005.

[21] G. Manning, D. B. Whyte, R. Martinez, T. Hunter, and S. Sudarsanam. The protein kinase complement of the human genome. Science, 298(5600):1912-1934, 2002.

[22] B. Alberts, A. Johnson, J. Lewis, M. Raff, K. Roberts, and P. Walter. Molecular Biology of the cell. Garland Science., 5 ed. edition, Nov. 2007.

[23] S. Li, M. Kim, Y. L. Hu, S. Jalali, D. D. Schlaepfer, T. Hunter, S. Chien, and J. Y. Shyy. Fluid shear stress activation of focal adhesion kinase. linking to mitogenactivated protein kinase. J. Biol. Chem., 272(48):30455-30462, 1997.

[24] S. Li, P. Butler, Y Wang, Y. Hu, D. Han, S. Usami, J. Guan, and S. Chien. The role of the dynamics of focal adhesion kinase in the mechanotaxis of endothelial cells. Proc. Natl. Acad. Sci. U. S. A., 99(6):3546-3551, 2002.

[25] W. J. Polacheck, A. E. German, A. Mammoto, D. E. Ingber, and R. D. Kamm. Mechanotransduction of fluid stresses governs 3d cell migration. Proc. Natl. Acad. Sci. U. S. A., 111(7):2447-2452, 2014.

[26] S. K. Hanks, M. B. Calalb, M. C. Harper, and S. K. Patel. Focal adhesion proteintyrosine kinase phosphorylated in response to cell attachment to fibronectin. Proc. Natl. Acad. Sci. U. S. A., 89(18):8487-91, 1992. 
[27] D. Ilić, C. H. Damsky, and T. Yamamoto. Focal ahesion kinase: at the crossroads of signal transduction. J. Cell Sci., 110(4):401-407, 1997.

[28] D. D. Schlaepfer, C. R. Hauck, and D. J. Sieg. Signaling through focal adhesion kinase. Prog, Biophys. Mol. Biol., 71(3-4):435-478, 1999.

[29] V. M. Golubovskaya, F. A. Kweh, and W. G. Cance. Focal adhesion kinase and cancer. Histol. Histopathol., 24(4):503-10, 2009.

[30] K. Vadali, X. Cai, and M. D. Schaller. Focal adhesion kinase: an essential kinase in the regulation of cardiovascular functions. IUBMB Life, 59(1):709-716, 2007.

[31] H. B. Wang, M. Dembo, S. K. Hanks, and Y. Wang. Focal adhesion kinase is involved in mechanosensing during fibroblast migration. Proc. Natl. Acad. Sci. U. S. A., 98(20):11295-11300, 2001.

[32] L. S. Chaturvedi, H. M. Marsh, and M. D. Basson. Src and focal adhesion kinase mediate mechanical strain-induced proliferation and erk1/2 phosphorylation in human h441 pulmonary epithelial cells. Am. J. Physiol., Cell Physiol., 292(5):C1701C1713, 2007.

[33] J. Seong, A. Tajik, J. Sun, J. L. Guan, M. J. Humphries, S. E. Craig, A. Shekaran, A. J. García, S. Lu, M. Z. Lin, N. Wang, and Y. Wang. Distinct biophysical mechanisms of focal adhesion kinase mechanoactivation by different extracellular matrix proteins. Proc. Natl. Acad. Sci. U. S. A., 110(48):19372-19377, 2013.

[34] MD. Schaller, JD. Hildebrand, JD Shannon, JW. Fox, RR. Vines, and JT. Parsons. Autophosphorylation of the focal adhesion kinase, pp125fak, directs sh2-dependent binding of pp60src. Mol.Cell.Biol., 14(3):1680-1688, 1994.

[35] M. B. Calalb, T. R. Polte, and S. K. Hanks. Tyrosine phosphorylation of focal adhesion kinase at sites in the catalytic domain regulates kinase activity: a role for src family kinase. Mol. Cell. Biol., 15(2):954-963, 1995.

[36] D. Lietha, X. Cai, D. F. Ceccarelli, Y. Li, M. D. Schaller, and M. J. Eck. Structural basis for the autoinhibition of focal adhesion kinase. Cell, 129(6):1177-1187, 2007.

[37] F. S David, P. E. Zage, and E. E. Marcantonio. Integrins interact with focal adhesions through multiple distinct pathways. J. Cell. Physiol., 181(1):74-82, 1999.

[38] S. Y. Chen and H. C. Chen. Direct interaction of focal adhesion kinase (fak) with met is required for fak to promote hepatocyte growth factor-induced cell invasion. Mol. Cell. Biol., 26(13):5155-5167, 2006.

[39] I. Plaza-Menacho, A. Morandi, L. Mologni, P. Boender, C. Gambacorti-Passerini, A. I. Magee, R. M. Hofstra, P. Knowles, N. Q. McDonald, and C. M. Isacke. Focal adhesion kinase(fak) binds ret kinase via its ferm domain, priming a direct and reciprocal ret-fak transactivation mechanism. J. Biol. Chem., 286(19):17292-17302, 2011. 
[40] D. J. Sieg, C. R. Hauck, D. K. KLingbeil, E. Schaefer, D. H. Damsky, and D. D. Schlaepfer. Fak integrates growth-factor and integrin signals to promote cell migration. Nat. Cell Biol., 2(5):249-256, 2000.

[41] G. M. Goñi, C. Epifano, J. Boskovic, M. Camacho-Artacho, J. Zhou, A. Bronowska, M.T. Martín, M. J. Eck, L. Kremer, F. Gräter, F. L. Gervasio, M. Perez-Moreno, and D. Lietha. Pi $(4,5) \mathrm{p} 2$ triggers activation of focal adhesion kinase by inducing clustering and conformational changes. Proc. Natl. Acad. Sci. USA., in print, 2014.

[42] X Cai, D. Lietha, D. F. Ceccarelli, A. V. Karginov, Z. Rajfur, K. Jacobson, K. M. Hahn, M. J. Eck, and M. D. Schaller. Spatial and temporal regulation of focal adhesion kinase activity in living cells. Mol. Cell. Biol., 28(1):201-214, 2008.

[43] N. Thapa and R. A. Anderson. Pip2 signaling, an integrator of cell polarity and vesicle trafficking in directionally migrating cells. Cell Adh. Migr., 6(5):409-412, 2012.

[44] D. A. Linseman, S. D. Sorensen, and S. K. Fisher. Attenuation of focal adhesion kinase signalling following depletion of agonist-sensitive pools of phosphatidylinositol 4,5-bisphosphate. J. Neurochem., 73(5):1933-1944, 1999.

[45] S. K. Mitra, D. A. Hanson, and D. D. Schlaepfer. Focal adhesion kinase: in command and control of cell motility. Nat. Rev. Mol. Cell Biol., 6(1):56-68, 2005.

[46] C. Baldauf, R. Schneppenheim, W. Stacklies, T. Obser, A. Pieconka, S. Schneppenheim, U. Budde, J. Zhou, and Gräter F. Shear-induced unfolding activates von willebrand factor a2 domain for proteolysis. J. Thromb. Haemost., 7(12):2096-2105, 2009.

[47] F. Gräter, J. Shen, H. Jiang, M. Gautel, and H. Grubmüller. Mechanically induced titin kinase activation studied by force-probe molecular dynamics simulations. Biophys. J., 88(2):790-804, 2005.

[48] D. W. Dumbauld, H. Shin, N. D. Gallant, K. E. Michael, H. Radhakrishna, and A. J. Garcia. Contractility modulates cell adhesion strengthening through focal adhesion kinase and assembly of vinculin-containing focal adhesions. J. Cell. Physiol., 223(3):746-756, 2010.

[49] W. Li and F. Gräter. Atomistic evidence of how force dynamically regulates thiol/disulfide exchange. J. Am. Chem. Soc., 132(47):16790-16795, 2010.

[50] OK. Dudko, G. Hummer, and A. Szabo. Intrinsic rates and activation free energies from single-molecule pulling experiments. Phys. Rev. Lett., 96(10):108101, 2006.

[51] G. Hummer and A. Szabo. Kinetics from nonequilibrium single-molecule pulling experiments. Biophys. J., 85(1):5-15, 2003.

[52] J-P. Ryckaert, G. Ciccotti, and H. J. C. Berendsen. Numerical integration of the cartesian equations of motion of a system with constraints: molecular dynamics of n-alkanes. J. Comput. Phys., 23(3):327-341, 1976. 
[53] B. Hess, H. Bekker, and J. G. E. M. Berendsen, J. C. und Fraaije. Lincs: a linear constraint solver for molecular simulations. J. Comput. Chem., 18:1463-1472, 1997.

[54] A. Cheng and K. M. Jr. Merz. Application of the nosé-hoover chain algorithm to the study of protein dynamics. J. Phys. Chem., 100(5):1927-1937, 1996.

[55] SH. Lee, K. Palmo, and S. Krimm. New out-of-plane angle and bond angle internal coordinates and related potential energy functions for molecular mechanics and dynamics simulations. J. Comput. Chem., 20(10):1067-1084, 1999.

[56] H. J. C. Berendsen, JR. Grigera, and T. P. Straatsma. The missing term in effective pair potentials. J. Phys. Chem., 91(24):6269-6271, 1987.

[57] W. L. Jorgensen, J. Chandrasekhar, J. D. Madura, R. W. Impey, and M. L. Klein. Comparison of simple potential functions for simulating liquid water. J. Chem. Phys., 79:926-935, 1983.

[58] B. R. Brooks, R. E. Bruccoleri, B. D. Olafson, D. J. States, S. Swaminathan, and M. Karplus. Charmm: A program for macromolecular energy, minimization, and dynamics calculations. J. Comput. Chem., 4(2):187-217, 1983.

[59] W. D. Cornell, P. Cieplak, C. I. Bayly, I. R. Gould, K. M. Jr. Merz, D. M. Ferguson, D. C. Spellmeyer, T. Fox, J. W. Caldwell, and P. A. Kollman. A second generation force field for the simulation of proteins, nucleic acids, and organic molecules. J. Am. Chem. Soc., 117:5179-5197, 1995.

[60] M. Christen, P. H. Hünenberger, D. Bakowies, R. Baron, R. Bürgi, D. P. Geerke, T. N. Heinz, M. A. Kastenholz, V. Kräutler, C. Oostenbrink, C. Peter, D. Trzesniak, and W. F. van Gunsteren. The gromos software for biomolecular simulation: Gromos05. J. Comput. Chem., 26(16):1719-1751, 2005.

[61] B. Hess, C. Kutzner, D. van der Spoel, and E. Lindahl. Gromacs 4: Algorithms for highly efficient, load-balanced, and scalable molecular simulation. J. Chem. Theory Comput., 4:435-447, 2008.

[62] W. L. Jorgensen, D. S. Maxwell, and J. Tirado-Rives. Development and testing of the opls all-atom force field on conformational energetics and properties of organic liquids. J. Am. Chem. Soc., 118(45):11225-11236, 1996.

[63] M. W. Mahoney and W. L. Jorgensen. A five-site model liquid water and the reproduction of the density anomaly by rigid, non-polarizable models. J. Chem. Phys., 112:8910-8922, 2000.

[64] R. W. Pastor and A. D. Jr. MacKerell. Development of the charmm force field for lipids. J. Phys. Chem. Lett., 2(13):1526-1532, 2011.

[65] H. Grubmüller, B. Heymann, and P. Tavan. Ligand binding: Molecular mechanics calculation of the streptavidin-biotin rupture force. Science, 271:997-999, 1996.

[66] S. Izrailev, S. Stepaniants, B. Isralewitz, D. Kosztin, H. Lu, F. Molnar, W. Wriggers, and K. Schulten. Computational Molecular Dynamics: Challenges, Methods, Ideas, volume 4 of Lecture Notes in Computational Science and Engineering., chapter Steered Molecular Dynamics., pages 39-65. Springer-Verlag, Berlin, 1998. 
[67] A. Raval, S. Piana, M. P. Eastwood, R. O. Dror, and D. E. Shaw. Refinement of protein structure homology models via long, all-atom molecular dynamics simulations. Proteins: Struct., Funct., Bioinf., 80(8):2071-2079, 2012.

[68] W. L. Jorgensen. Rusting of the lock and key model for protein-ligand binding. Science., 254(5034):954-955, 1991.

[69] B. Q. Wei, L. H. Weaver, A. M. Ferrari, B. W. Matthews, and B. K. Shoichet. Testing a flexible-receptor docking algorithm in a model binding site. J. Mol. Biol., 337(5):1161-1182, 2004.

[70] O. F. Lange, N. A. Lakomek, C. Farés, GF. Schröder, K. F. Walter, S. Becker, J. Meiler, H Grubmüller, C. Griesinger, and B. L. de Groot. Recognition dynamics up to microseconds revealed from an rdc-derived ubiquitin ensemble in solution. Science, 320(5882):1471-1475, 2008.

[71] B. Mukesh and K. Rakesh. Molecular docking: a review. IJRAP, 2(6):1746-1751, 2011.

[72] W. Stacklies, C. Seifert, and F. Gräter. Implementation of force distribution analysis for molecular dynamics simulations. BMC Bioinformatics, 12:101, 2011.

[73] B. I. Costescu and F. Gräter. Time-resolved force distribution analysis. BMC Biophys., 6(1):5, 2013.

[74] B. I. Costescu and F. Gräter. Graphene mechanics: Ii. atomic stress distribution during indentation until rupture. Phys. Chem. Chem. Phys., 16(24):12582-12590, 2014.

[75] A. Amadei, A. B. Linssen, and H. J. Berendsen. Essential dynamics of proteins. Proteins, 17(4):412-425, 1993.

[76] T. Krivobokova, R. Briones, J. S. Hub, A. Munk, and B. L. de Groot. Partial leastsquares functional mode analysis: application to the membrane proteins aqp1, aqy1, and clc-ec1. Biophys. J., 103(4):786-796, 2012.

[77] S. Hoops, S. Sahle, R. Gauges, C. Lee, J. Pahle, N. Simus, M. Singhal, L. Xu, P. Mendes, and U. Kummer. Copasi: a complex pathway simulator. Bioinformatics, 22:3067-3074, 2006.

[78] C. E. Turner. Paxillin and focal adhesion signalling. Nat. Cell Biol., 2(12):E231E236, 2000.

[79] MD. Schaller. Paxillin: a focal adhesion-associated adaptor protein. Oncogene, 20(44):6459-6472, 2001.

[80] P. P. Eleniste and A. Bruzzaniti. Focal adhesion kinases in adhesion structures and disease. J. Signal Transduct., 2012:296450, 2012. 
[81] U. Pieper, N. Eswar, H. Braberg, M. S. Madhusudhan, F. P. Davis, A. C. Stuart, N. Mirkovic, A. Rossi, M. A. Marti-Renom, A. Fiser, B. Webb, D. Greenblatt, C. C. Huang, T. E. Ferrin, and A. Sali. Modbase, a database of annotated comparative protein structure models, and associated resources. Nucleic Acids Res., 32(Dastbase issue):D217-2122, 2004.

[82] E. Vanquelef, S. Simon, G. Marquant, E. Garcia, G. Klimerak, J. C. Delepine, P. Cieplak, and F.-Y Dupradeau. R.e.d. server: a web service for deriving resp and esp charges and building force field libraries for new molecules and molecular fragments. Nucleic Acids Res., 39:W511-W517, 2011.

[83] F. Y. Dupradeau, A. Pigache, T. Zaffran, C. Savineau, R. Lelong, N. Grivel, D. Lelong, W. Rosanski, and P. Cieplak. The r.e.d. tools: advances in resp and esp charge derivation and force field library building. Phys. Chem. Chem. Phys., 12:7821-7839, 2010 .

[84] M. J. Frisch, G. W. Trucks, H. B. Schlegel, G. E. Scuseria, M. A. Robb, J. R. Cheeseman, G. Scalmani, V. Barone, B. Mennucci, G. A. Petersson, H. Nakatsuji, M. Caricato, X. Li, H. P. Hratchian, A. F. Izmaylov, J. Bloino, G. Zheng, J. L. Sonnenberg, M. Hada, M. Ehara, K. Toyota, R. Fukuda, J. Hasegawa, M. Ishida, T. Nakajima, Y. Honda, O. Kitao, H. Nakai, T. Vreven, J. A. Montgomery, Jr., J. E. Peralta, F. Ogliaro, M. Bearpark, J. J. Heyd, E. Brothers, K. N. Kudin, V. N. Staroverov, R. Kobayashi, J. Normand, K. Raghavachari, A. Rendell, J. C. Burant, S. S. Iyengar, J. Tomasi, N. Cossi, M.and Rega, J. M. Millam, M. Klene, J. E. Knox, J. B. Cross, V. Bakken, C. Adamo, J. Jaramillo, R. Gomperts, R. E. Stratmann, O. Yazyev, A. J. Austin, R. Cammi, C. Pomelli, J. W. Ochterski, R. L. Martin, K. Morokuma, V. G. Zakrzewski, G. A. Voth, P. Salvador, J. J. Dannenberg, S. Dapprich, A. D. Daniels, J. B. Foresman, J. V. Ortiz, J. Cioslowski, and D. J. Fox. Gaussian 09. Wallingford, CT, 2009.

[85] S. McLaughlin, J. Wang, A. Gambhir, and D. Murray. Pip(2) and proteins: interactions, organization, and information flow. Annu. Rev. Biophys. Biomol. Struct., 31:151-175, 2002.

[86] P. T. Lang, S. R. Brozell, S. Mukherjee, E. F. Pettersen, E. C. Meng, V. Thomas, R. C. Rizzo, D. A. Case, T. L. James, and I. D. Kuntz. Dock 6: combining techniques to model rna-small molecule complexes. RNA, 15:1219, 2009.

[87] M. Parrinello and A. Rahman. Polymorphic transitions in single crystals: A new molecular dynamics method. J. Appl. Phys., 52:7182, 1981.

[88] T. Darden, D. York, and L. Pedersen. Particle mesh ewald: An $n \cdot \log (n)$ method for ewald sums in large systems. J. Chem. Phys., 98:10089-10092, 1993.

[89] M. A. Balsera, W. Wriggers, Y. Oono, and K. Schulten. Principal component analysis and long time protein dynamics. J. Phys. Chem., 100:2567-2572, 1996.

[90] C. Seifert and F. Graeter. Force distribution reveals signal transduction in e. coli hsp90. Biophys J., 103(10):2195-2202, 2012. 
[91] T. H. Chen, P. C. Chan, C. L. Chen, and H. C. Chen. Phosphorylation of focal adhesion kinase on tyrosine 194 by met leads to its activation through relief of autoinhibition. Oncogene, 30(2):153-166, 2011.

[92] Z. Palmai, C. Seifert, F. Gräter, and E. Balog. An allosteric signaling pathway of human 3- phosphoglycerate kinase from force distribution analysis. PLoS Comput. Biol., 10(1):e1003444, 2014.

[93] H. T. Young, S. A. Edwards, and F. Gräter. How fast does a signal propagate through proteins? PLoS One, 8(6):e64746, 2013.

[94] A. del Rio, R. Perez-Jimenez, R. Liu, P. Roca-Cusachs, J. M. Fernandez, and M. P. Sheetz. Stretching single talin rod molecules activates vinculin binding. Science, 323(5914):638-641, 2009.

[95] C. Grashoff, B. D. Hoffman, M. D. Brenner, R. Zhou, M. Parsons, M. T. Yang, M. A. McLean, S. G. Sligar, C. S. Chen, T. Ha, and M. A. Schwartz. Measuring mechanical tension across vinculin reveals regulation of focal adhesion dynamics. Nature, 466(7303):263-266, 2010.

[96] Y. Yano, J. Geibel, and B. Sumpio. Tyrosine phosphorylation of pp125fak and paxillin in aortic endothelial cells induced by mechanical strain. Am. J. Physiol., 271:C635-C649, 1996.

[97] D. Tang, D. Mehta, and S. Gunst. Mechanosensitive tyrosine phosphorylation of paxillin and focal adhesion kinase in tracheal smooth muscle. Am. J. Physiol., 276:C250C258, 1999.

[98] R. Schmalzigaug, M. L. Garron, J. T. Roseman, Y. Xing, C. E. Davidson, S. T. Arold, and R. T. Premont. Git1 utilizes a focal adhesion targeting-homology domain to bind paxillin. Cell, 19(8):1733-1744, 2007.

[99] G. Di Paolo, L. Pellegrini, K. Letinic, G. Cestra, R. Zonc, S. Voronov, S. Chang, J. Guo, M. R. Wenk, and P. De Camilli. Recruitment and regulation of phosphatidylinositol phosphate kinase type 1 gamma by the ferm domain of talin. Nature, 420(6911):85-89, 2002.

[100] K. Ling, R. L. Doughman, A. J. Firestone, M. W. Bunce, and R. A. Anderson. Type i gamma phosphatidylinositol phosphate kinase targets and regulates focal adhesions. Nature, 420(6911):89-93, 2002.

[101] R. A Anderson and Marchesi V. T. Regulation of the association of membrane skeletal protein 4.1 with glycophorin by a polyphosphoinositide. Nature, 318(6043):295$298,1985$.

[102] S. McLaughlin and D. Murray. Plasma membrane phosphoinositide organization by protein electrostatics. Nature, 438(7068):605-611, 2005.

[103] D. J. James, C. Khodthong, J. A. Kowalchyk, and T. F. Martin. Phosphatidylinositol 4,5-bisphosphate regulates snare-dependent membrane fusion. J. Cell Biol., 182(2):355-366, 2008. 
[104] T. Laux, K. Fukami, M. Thelen, T. Golub, D. Frey, and P. Caroni. Gap43, marcks, and cap23 modulate pi $(4,5) \mathrm{p}(2)$ at plasmalemmal rafts, and regulate cell cortex actin dynamics through a common mechanism. J. Cell Biol., 149(7):1455-1472, 2000.

[105] I. Levental, F. J. Byfield, P. Chowdhury, F. Gai, T. Baumgart, and P. A. Janmey. Cholesterol-dependent phase separation in cell-derived giant plasma-membrane vesicles. Biochem. J., 424(2):163-167, 2009.

[106] K. Carvalho, L. Ramos, C. Roy, and C. Picart. Giant unilamellar vesicles containing phosphatidylinositol $(4,5)$ bisphosphate: characterization and functionality. Biophys. J., 95(9):4348-4360, 2008.

[107] G. Giannone, P. Rondé, M. Gaire, J. Beaudouin, J. Haiech, J. Ellenberg, and K. Takeda. Calcium rises locally trigger focal adhesion disassembly and enhance residency of focal adhesion kinase at focal adhesions. J. Biol. Chem., 279(27):2871528723, 2004.

[108] E. von Castelmur, J. Strümpfer, B. Franke, J. Bogomolovas, S. Barbieri, H. Qadota, P. V. Konarev, D. I. Svergun, S. Labeit, G. M. Benian, K. Schulten, and O. Mayans. Identification of an n-terminal inhibitory extension as the primary mechanosensory regulator of twitchin kinase. Proc. Natl. Acad. Sci. U. S. A., 109(34):13608-13613, 2012.

[109] E. M. Puchner, A. Alexandrovich, A. L. Kho, U. H. Hensen, L. V. Schäfer, B. Brandmeier, F. Gräter, H. Grubmüller, H. E. Gaub, and M. Gautel. Mechanoenzymatics of titin kinase. Proc. Natl. Acad. Sci. U. S. A., 105(36):13385-13390, 2008.

[110] S. Lange, F. Xiang, A. Yakovenko, A. Vihola, P. Hackman, E. Rostkova, J. Kristensen, B. Brandmeier, G. Franzen, B. Hedberg, L. G. Gunnarsson, S. M. Hughes, S. Marchand, T. Sejersen, I. Richard, L. Edström, E. Ehler, B. Udd, and M. Gautel. The kinase domain of titin controls muscle gene expression and protein turnover. Science, 308(5728):1599-1603, 2005.

[111] G. Bussi, D. Donadio, and M. Parrinello. Canonical sampling through velocity rescaling. J. Chem. Phys., 126(1):014101, 2007.

[112] E. L. Wu, X. Cheng, S. Jo, H. Rui, K. C. Song, E. M. Dávila-Contreras, Y. Qi, J. Lee, V. Monje-Galvan, R. M. Venable, J. B. Klauda, and W. Im. Charmm-gui membrane builder toward realistic biological membrane simulations. J. Comput. Chem., 35(27):1997-2004, 2014.

[113] S. Pronk, S. Páll, R. Schulz, P. Larsson, P. Bjelkmar, R. Apostolov, M. R. Shirts, J. C. Smith, P. M. Kasson, D. van der Spoel, B. Hess, and E. Lindahl. Gromacs 4.5: a high-throughput and highly parallel open source molecular simulation toolkit. Bioinformatics, 29(7):845-854, 2013.

[114] J. C. Fuller, M. Martinez, and R. C. Wade. On calculation of the electrostatic potential of a phosphatidylinositol phosphate-containing phosphatidylcholine lipid membrane accounting for membrane dynamics. PLoS One, 9(8):e104778, 2014. 
[115] W. L. Jorgensen. Quantum and statistical mechanical studies of liquids. 10. transferable intermolecular potential functions for water, alcohols, and ethers. application to liquid water. J. Am. Chem. Soc., 103:335-340, 1981.

[116] E. R. Cruz-Chu and K. Schulten. Computational microscopy of the role of protonable surface residues in nanoprecipitation oscillations. ACS Nano., 4(8):4463-4474, 2010.

[117] D. Lupyan, M. Mezei, DE. Logothetis, and R. Osman. A molecular dynamics investigation of lipid bilayer perturbation by pip2. Biophys. J., 98(2):240-247, 2010.

[118] Y. H. Ellenbroek, W. G.and Wang, D. A. Christian, D. E. Discher, P. A. Janmey, and A. J. Liu. Divalent cation-dependent formation of electrostatic pip2 clusters in lipid monolayers. Biophys. J., 101(9):2178-2184, 2011.

[119] M. Rief, M. Gautel, F. Oesterhelt, J. M. Fernandez, and H. E. Gaub. Reversible unfolding of individual titin immunoglobulin domains by afm. Science, 276(5315):1109-1112, 1997.

[120] D. H. Nguyen, D. J. Webb, A. D. Catling, Q. Song, A. Dhakephalkar, M. J. Weber, K. S. Ravichandran, and S. L. Gonias. Urokinase-type plasminogen activator stimulates the ras/extracellular signal-regulated kinase (erk) signaling pathway and mcf-7 cell migration by a mechanism that requires focal adhesion kinase, src, and shc. rapid dissociation of grb2/sps-shc complex is associated with the transient phosphorylation of erk in urokinase-treated cells. J. Biol. Chem., 275(25):19382, 193882000.

[121] DD. Schlaepfer, KC. Jones, and T. Hunter. Multiple grb2-mediated integrinstimulated signaling pathways to erk $2 /$ mitogen-activated protein kinase: summation of both c-src- and focal adhesion kinase-initiated tyrosine phosphorylation events. Mol Biol Cell., 18(5):2571-2585, 1998.

[122] M. Toutant, A. Costa, J. M. Studler, G. Kadaré, M. Carnaud, and J. A. Girault. Alternative splicing controls the mechanisms of fak autophosphorylation. Mol. Cell. Biol., 22(22):7731-7743., 2002.

[123] W. Liang, K. Ren, F. Liu, W. Cui, Q. Wang, Z. Chen, and W. Fan. Periodic mechanical stress stimulates the fak mitogenic signal in rat chondrocytes through erk $1 / 2$ activity. Cell. Physiol. Biochem., 32(4):915-930, 2013.

[124] H. Lal, S. K. Verma, M. Smith, R. S. Guleria, G. Lu, D. M. Foster, and D. E. Dostal. Stretch-induced map kinase activation in cardiac myocytes: differential regulation through beta1-integrin and focal adhesion kinase. J. Mol. Cell. Cardiol., 43(2):137'47, 2007.

[125] K. Brami-Cherrier, N. Gervasi, D. Arsenieva, K. Walkiewicz, M. C. Boutterin, A. Ortega, P. G. Leonard, B. Seantier, L. Gasmi, T. Bouceba, G. Kadaré, J. A. Girault, and S. T. Arold. Fak dimerization controls its kinase-dependent functions at focal adhesions. EMBO J., 33(4):356-370, 2014.

[126] R. J. Boerner, D. B. Kassel, S. C. Barker, B. Ellis, P. DeLacy, and W. B. Knight. Correlation of the phosphorylation states of pp60c-src with tyrosine kinase activity: the intramolecular py530-sh2 complex retains significant activity if y419 is phosphorylated. Biochemistry, 35(29):9519-9525, 1996. 
[127] B. N. Kholodenko, O. V. Demin, G. Moehren, and J. B. Hoek. Quantification of short term signaling by the epidermal growth factor receptor. J. Biol. Chem., 274(42):30169-30181, 1999.

[128] M. Hatakeyama, S. Kimura, T. Naka, T. Kawasaki, N. Yumoto, M. Ichikawa, J. H. Kim, K. Saito, M. Saeki, M. Shirouzu, S. Yokoyama, and A. Konagaya. A computational model on the modulation of mitogen-activated protein kinase (mapk) and akt pathways in heregulin-induced erbb signalling. Biochem. J., 373(2):451-463, 2003.

[129] A. Koo, D. Nordsletten, R. Umeton, B. Yankama, S. Ayyadurai, G. García-Cardeña, and C. F. Jr. Dewey. In silico modeling of shear-stress-induced nitric oxide production in endothelial cells through systems biology. Biophys. J., 104(10):2295-2306, 2013.

[130] K. L. Yee, V. M. Weaver, and D. A. Hammer. Integrin-mediated signalling through the map-kinase pathway. IET Systems Biology, 2(1):8-15, 2008.

[131] M. Rief, J. Pascual, M. Saraste, and H. E. Gaub. Single molecule force spectroscopy of spectrin repeats: low unfolding forces in helix bundles. J. Mol. Biol., 286(2):553$561,1999$.

[132] X. Wang and T. Ha. Defining single molecular forces required to activate integrin and notch signaling. Science, 340(6135):991-994, 2013.

[133] B. Schoeberl, C. Eichler-Jonsson, E. D. Gilles, and G. Müller. Computational modeling of the dynamics of the map kinase cascade activated by surface and internalized egf receptors. Nat. Biotechnol., 20(4):370-375, 2002.

[134] S. Saleem, J. Li, S. P. Yee, G. F. Fellows, C. G. Goodyer, and R. Wang. Beta1 integrin/fak/erk signalling pathway is essential for human fetal islet cell differentiation and survival. J. Pathol., 219(2):182-192, 2009.

[135] S. Piana, K. Lindorff-Larsen, and Shaw D. E. Atomic-level description of ubiquitin folding. Proc. Natl. Acad. Sci. U. S. A., 110(15):5915-5920, 2013.

[136] F. Xia, D. Thirumalai, and Gräter F. Minimum energy compact structures in forcequench polyubiquitin folding are domain swapped. Proc. Natl. Acad. Sci. U. S. A., 108(17):6963-6968, 2011.

[137] J. T. Bullerjahn, S. Sturm, and K. Kroy. Theory of rapid force spectroscopy. Nat. Commun., 5:4463, 2014.

[138] M. A. Pufall and B. J. Graves. Autoinhibitory domains: modular effectors of cellular regulation. Annu. Rev. Cell Dev. Biol., 18:421-462, 2002. 